\title{
HOW HEALTHCARE PROFESSIONALS IN ACUTE CARE ENVIRONMENTS DESCRIBE PATIENT SAFETY: A CASE STUDY
}

\author{
By \\ JOANNA WAILLING
}

A thesis submitted to the Victoria University of Wellington in fulfilment of the requirements for the degree of

Master of

Health Research

Victoria University of Wellington New Zealand

2016 


\section{Abstract}

Patient safety has become an international healthcare priority over the past two decades. The prevailing approach to prevent harm in healthcare environments is the implementation of systems and structures that have made significant safety gains in high reliability organisations, such as aviation and nuclear power. However, similar safety improvements have not been realised in the healthcare environment. Studies suggest occupational culture is of importance, though our understanding of the relevance of safety subcultures is limited. This study explores how patient safety is described from the perspective of clinicians and organisational managers in an acute care hospital, using embedded case study design.

The case for this study was a New Zealand tertiary hospital. The emergency department and intensive care unit provided the settings for the embedded units. Three interviews with health care managers and six focus groups with nineteen doctors and nineteen nurses were undertaken. An interview guide, informed by the literature was used in data collection. Thematic data analysis was conducted within and across the case and embedded units. The theoretical concept of safety capability was developed from the data. Safety capability was defined as the ability to provide safe patient care and underpinned by the themes of resilient culture, and anticipation and vigilance.

A key finding of this research was that acute care environments have unique patient safety challenges, and these are influenced by complex factors. Patient safety was not assessed as being safe or unsafe, but rather perceived to exist across different levels of safety. Given this, healthcare professionals accept that some harm is inevitable in the healthcare setting. Doctors, nurses and managers understand and manage patient safety differently, and this affects how patient safety is addressed. This study identified anticipatory and vigilant systems are used to proactively manage risk by doctors and nurses, whereas incident reporting systems are used more by managers. 
Given the need to keep patients safe and avoid harm, more proactive patient safety systems are needed to manage patient safety in hospitals; this will require a paradigm shift away from current reactive safety systems. Proactive systems must be underpinned by a resilient patient safety culture that focuses on the right building blocks to produce balance of resources and targets and develop collaboration in organisations. This will bring about flexibility and stability to meet the complex conditions presented by acute care environments. 


\section{Dedication}

This thesis is dedicated to the patients whose names I have not forgotten but cannot document here

\&

Dr. Paul Wallman and the BSUH team.

You inspired me to consider if there is another way to ensure patients are at the heart of all we do.

"You can't get out of the current paradigm using the current model of thinking." (ICUSDR2) 


\section{Acknowledgements}

I have been very fortunate to be supported, encouraged and challenged by some very special people during the process of writing this thesis and am deeply indebted to my supervisors. To Dr. Brian Robinson: thank you for encouraging me to look at patient safety from multiple perspectives and consider an alternative approach. Most of all thank you for believing in me, I will be forever grateful for your time, energy and encouragement.

I wish to express my sincere appreciation to Professor Mo Coombs. You have inspired me to try research methods that have previously eluded me, and helped craft my writing style. The time and effort that has required cannot be underestimated. I will be eternally grateful for the interest and energy you have shown. Your patience, and your willingness to "hold up the mirror" has provided new insights into how to make a difference as a nursing leader, for this you have my sincere thanks.

I have worked with some incredible healthcare professionals, especially over the last few years, and their commitment and advocacy for patient safety has inspired me greatly. I must acknowledge Beelah Bleakley, Dr. Alex Psiridies, Michelle Halford and Dr. Iwona Storlarek. You are inspirational professionals and kind friends. For all you have done, and continue to do I am so very grateful.

To all the participants who took part in this study: Thank you for your candour and your willingness to share your thoughts and experiences. I hope this thesis describes the world as you view it, as was my primary intention.

My final words must be for my husband Matt, and my children Ollie and Imogen. Without your love and your willingness to give up hours of family time this research would never have been possible. Additionally, the small contribution I have been able to make to patient safety in my working life would not have happened without all you do and provide to support me at home. You are all incredible, and continue to teach me much about resilience. Thank you. 


\section{Table of Contents}

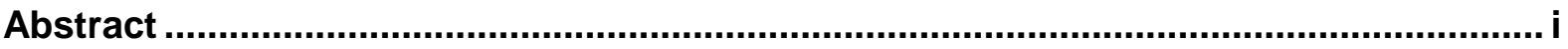

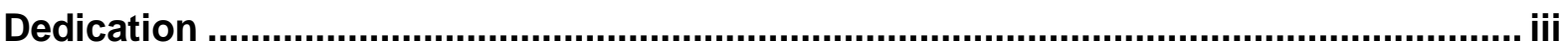

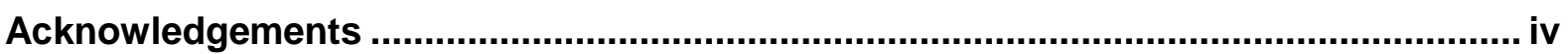

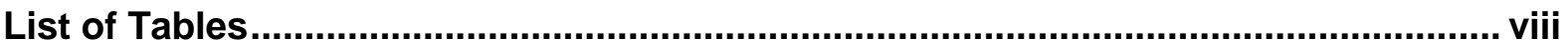

List of Figures ......................................................................................................... viii

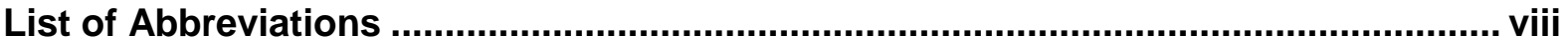

Patient safety: Lessons from a novice .................................................................... ix

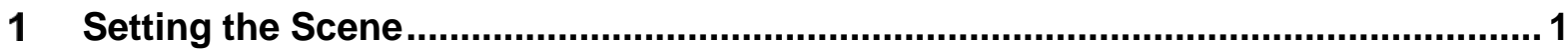

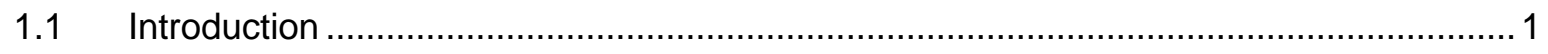

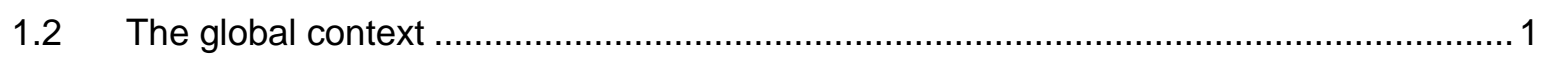

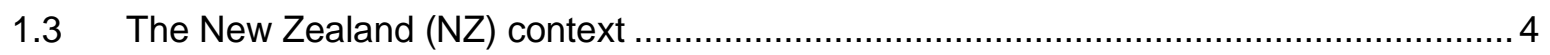

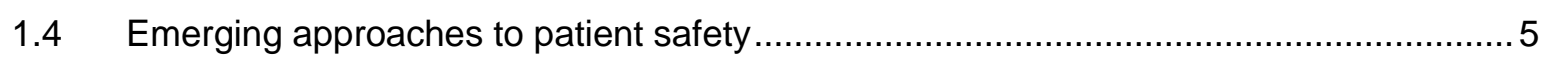

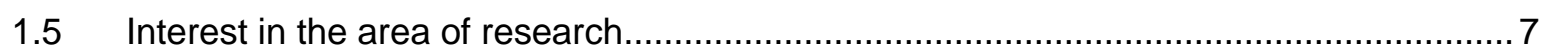

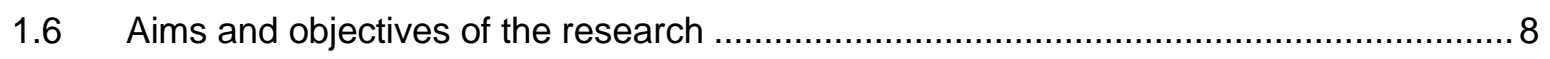

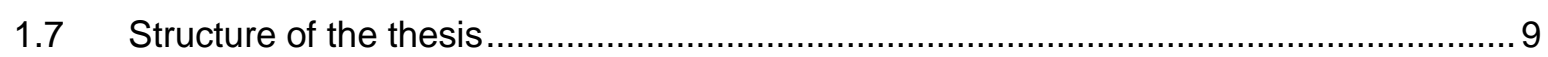

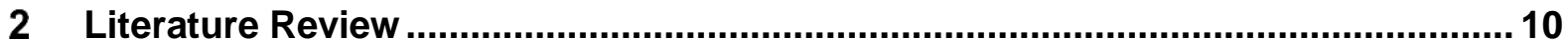

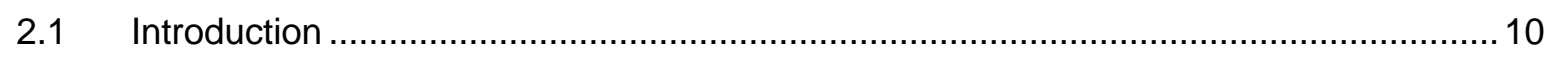

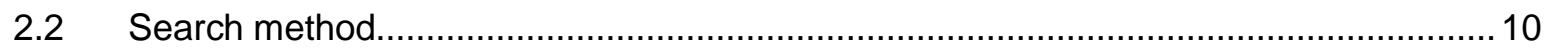

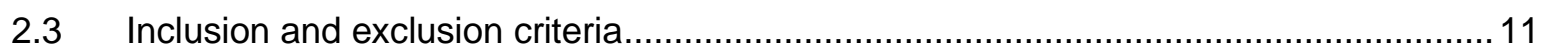

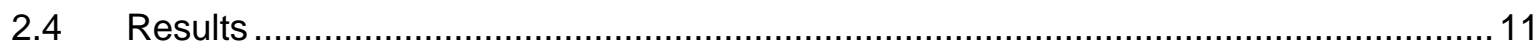

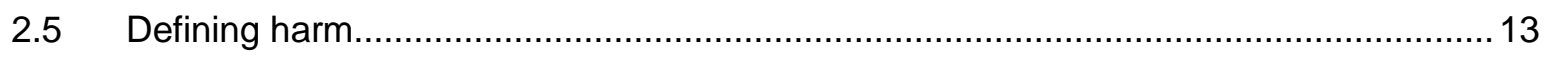

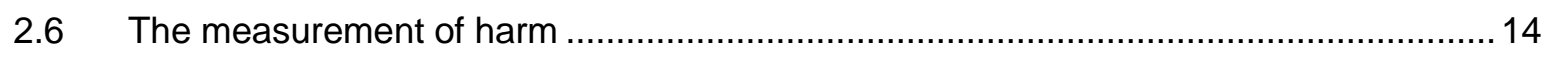

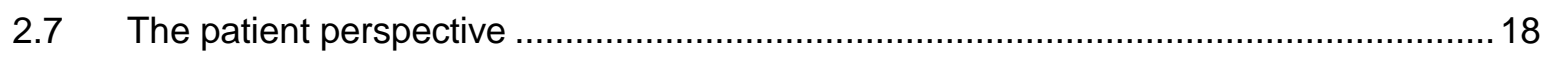

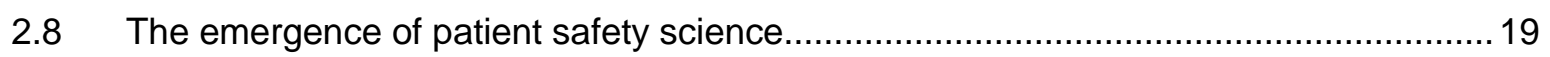

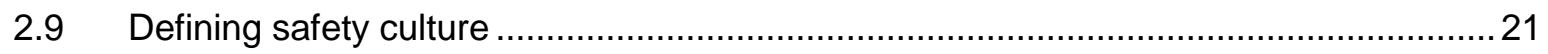

2.10 Significance of patient safety culture in the healthcare setting ......................................23

2.11 The multiple perspectives of patient safety culture .................................................. 24

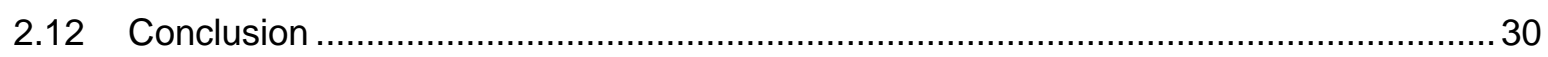

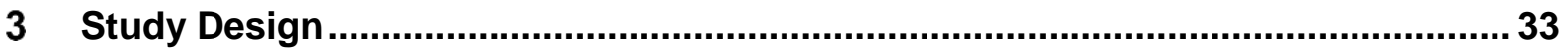

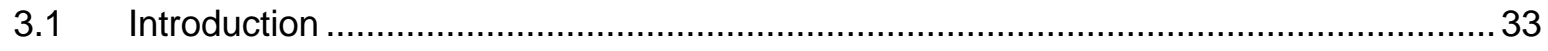

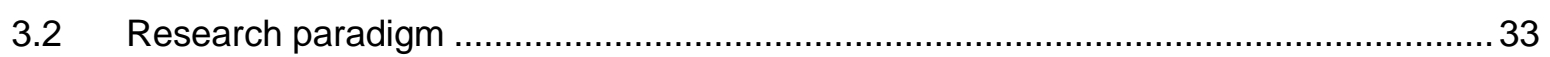

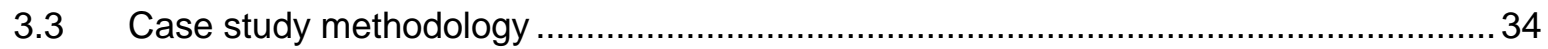

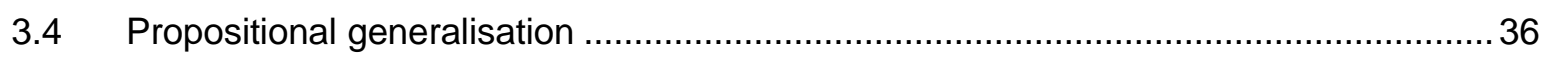

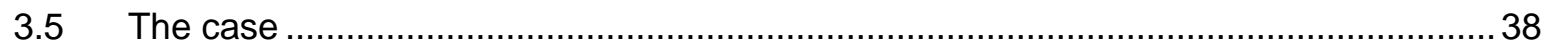

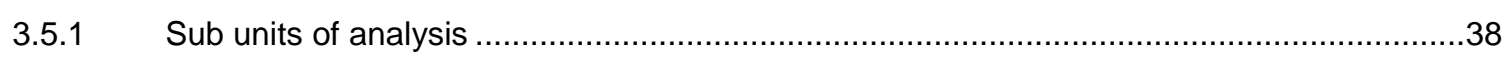




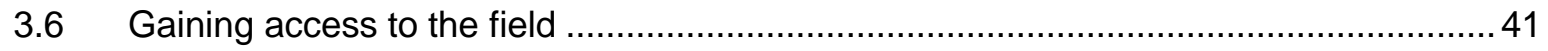

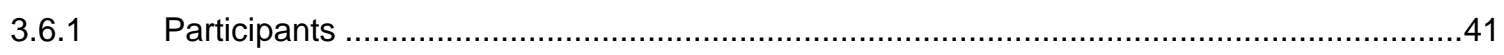

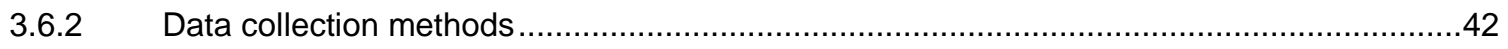

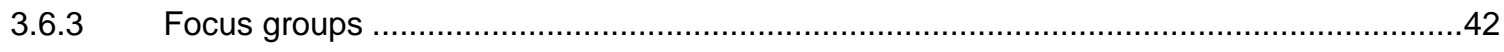

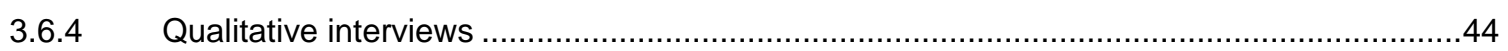

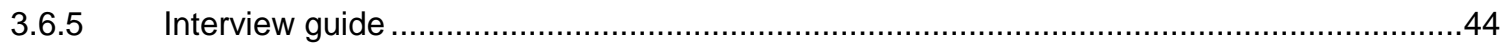

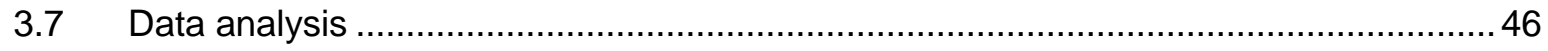

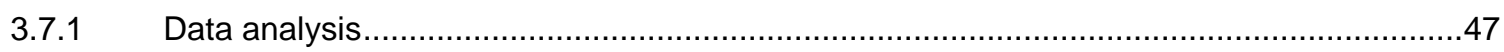

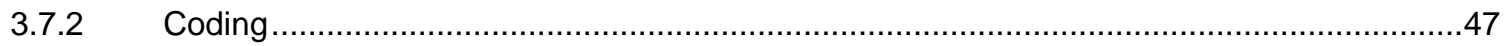

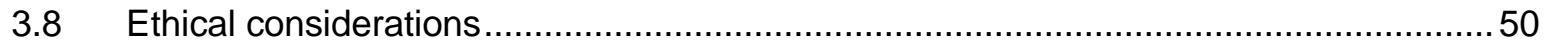

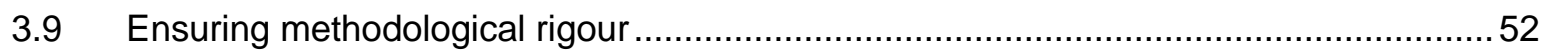

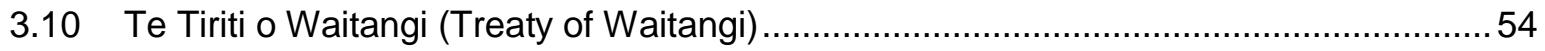

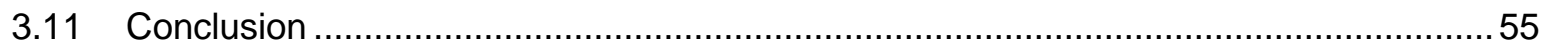

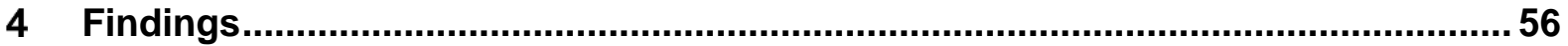

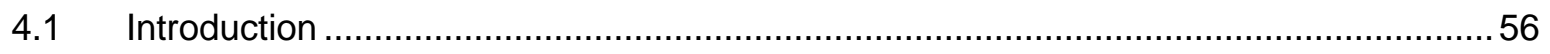

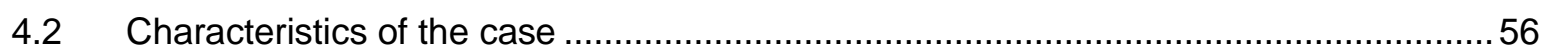

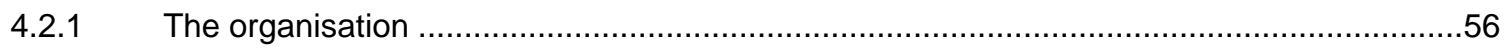

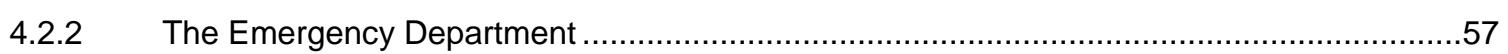

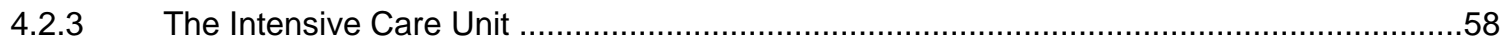

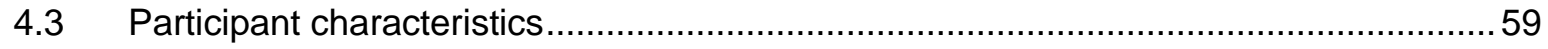

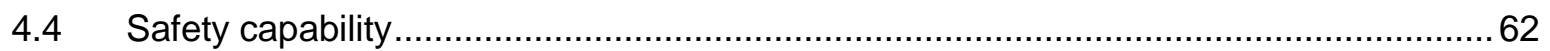

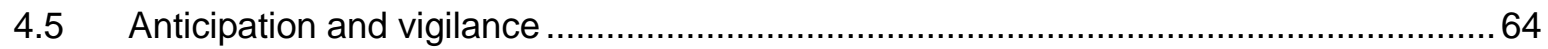

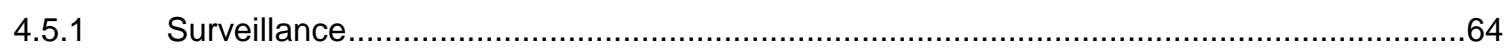

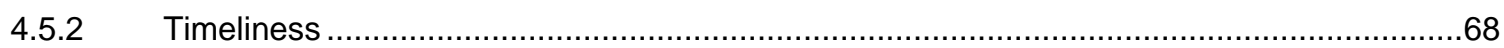

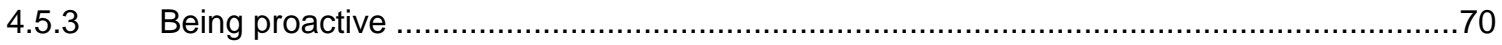

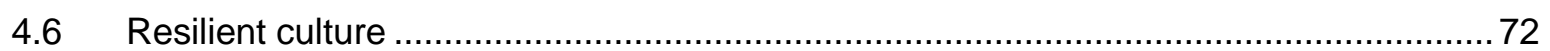

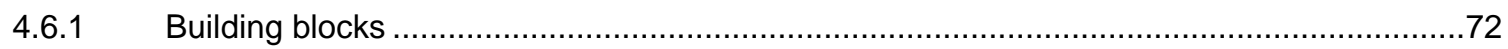

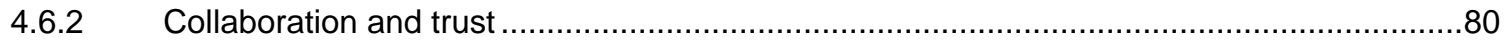

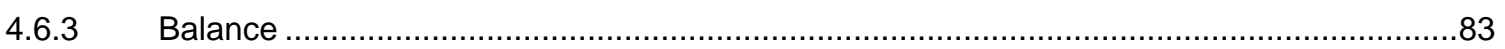

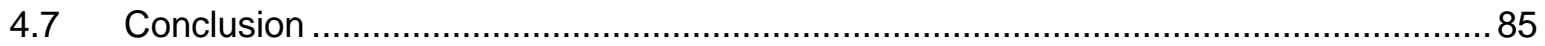

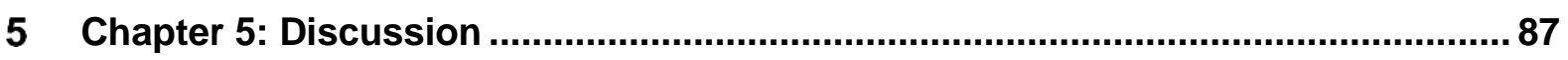

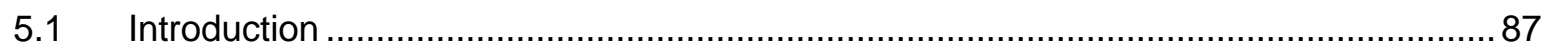

5.2 Proposition 1: Healthcare professionals embrace the concept of patient safety. Occupational groups describe the phenomenon differently. ............................................... 89

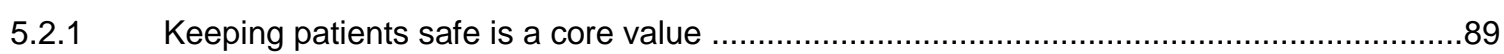

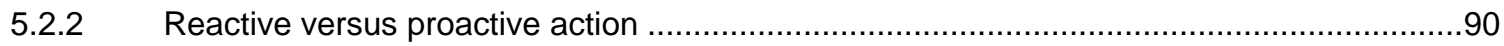

5.3 Proposition 2: Patient safety in acute care has unique challenges ...............................93

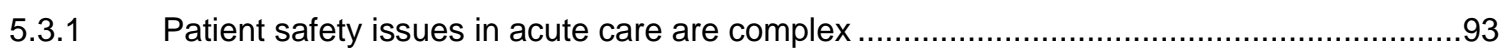

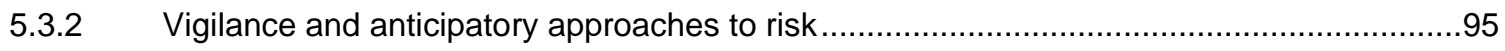

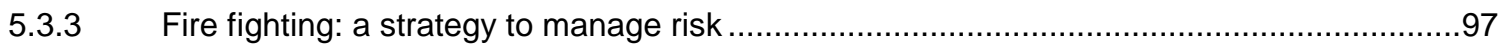




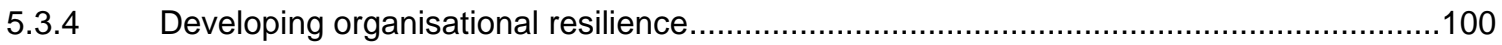

5.4 Proposition 3: Zero harm is unobtainable in healthcare environments ........................ 102

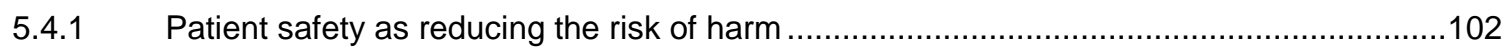

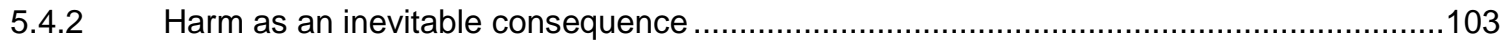

5.4.3 Risk assessment processes to mitigate iatrogenic harm ................................................104

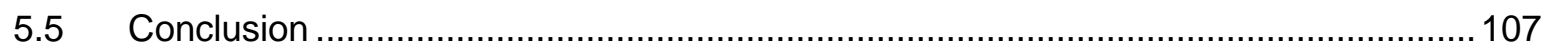

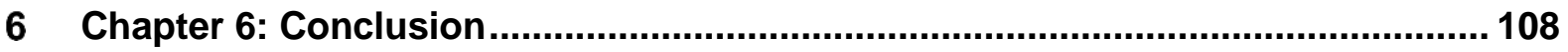

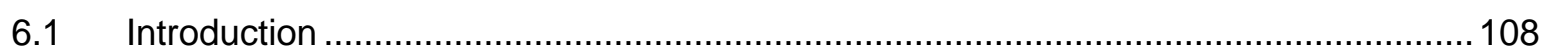

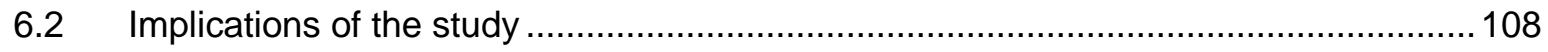

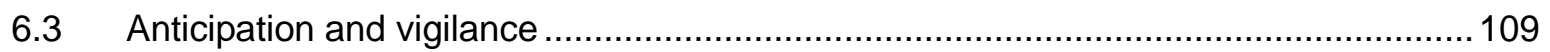

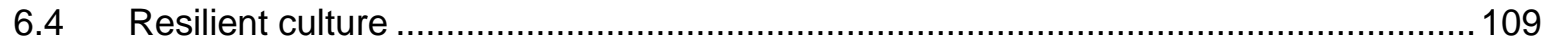

6.5 Strengths and limitations of the research ........................................................... 110

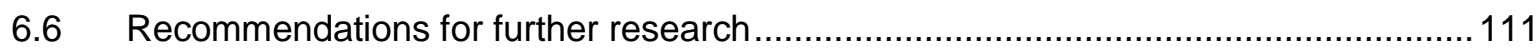

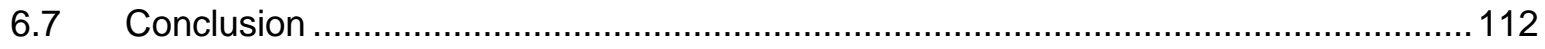

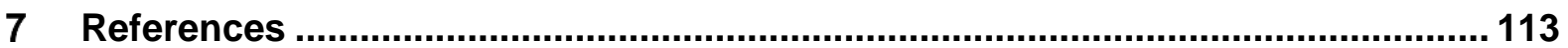

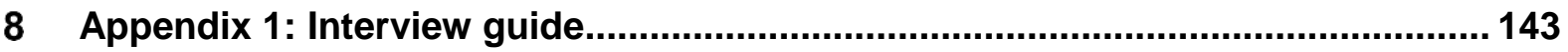

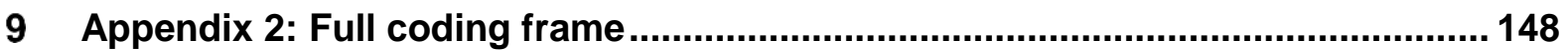

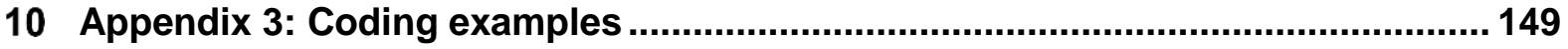

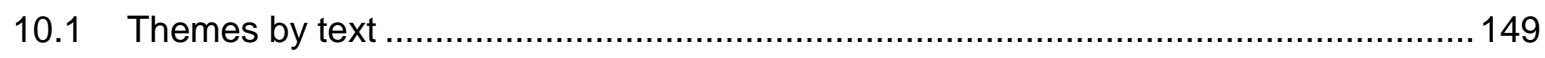

11 Appendix 4: Generating definitions from coding .............................................. 150

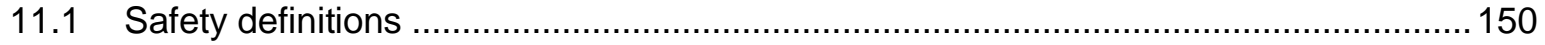

12 Appendix 5: Developing themes and sub themes .............................................. 151

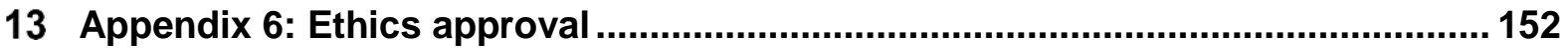

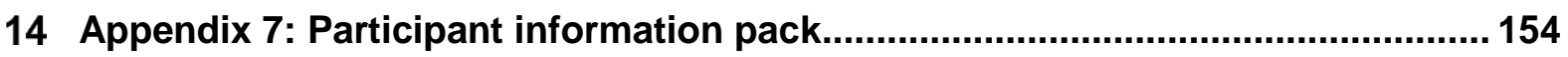

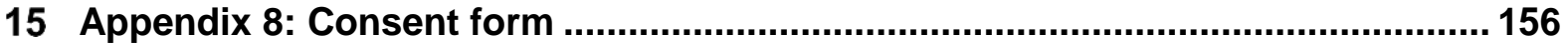

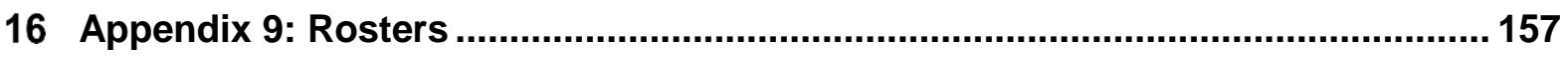




\section{List of Tables}

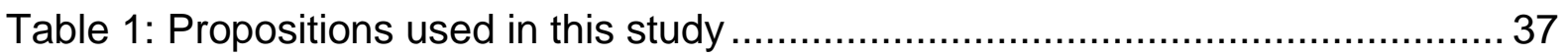

Table 2: Characteristics of participants in focus groups ....................................... 60

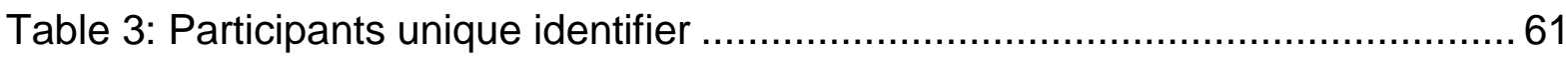

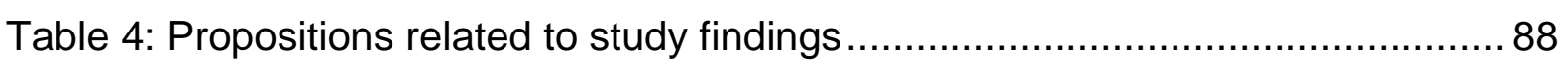

\section{List of Figures}

Figure 1: Embedded Cases - Units of Analysis …………………..................... 39

Figure 2: Embedded Cases - Sub Units of Analysis ........................................... 40

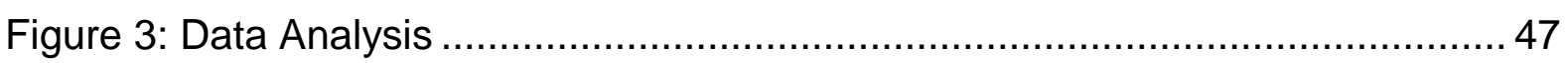

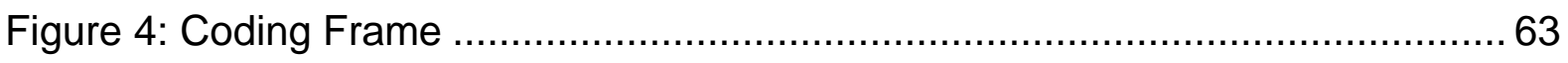

\section{List of Abbreviations}

\begin{tabular}{|l|l|}
\hline ED & Emergency Department \\
\hline EWS & Early Warning Score \\
\hline DH & Department of Health UK \\
\hline HDC & Health and Disability Commission NZ \\
\hline HQSC & Health Quality and Safety Commission NZ \\
\hline HRO & High Reliability Organisation \\
\hline ICU & Intensive Care Unit \\
\hline MH & Ministry of Health NZ \\
\hline IHI & Institute of Healthcare Improvement US \\
\hline RMO & Resident Medical Officer \\
\hline RN & Registered Nurse \\
\hline SMO & Senior Medical Officer \\
\hline TH & Tertiary Hospital \\
\hline WHO & World Health Organisation \\
\hline
\end{tabular}




\section{Patient safety: Lessons from a novice}

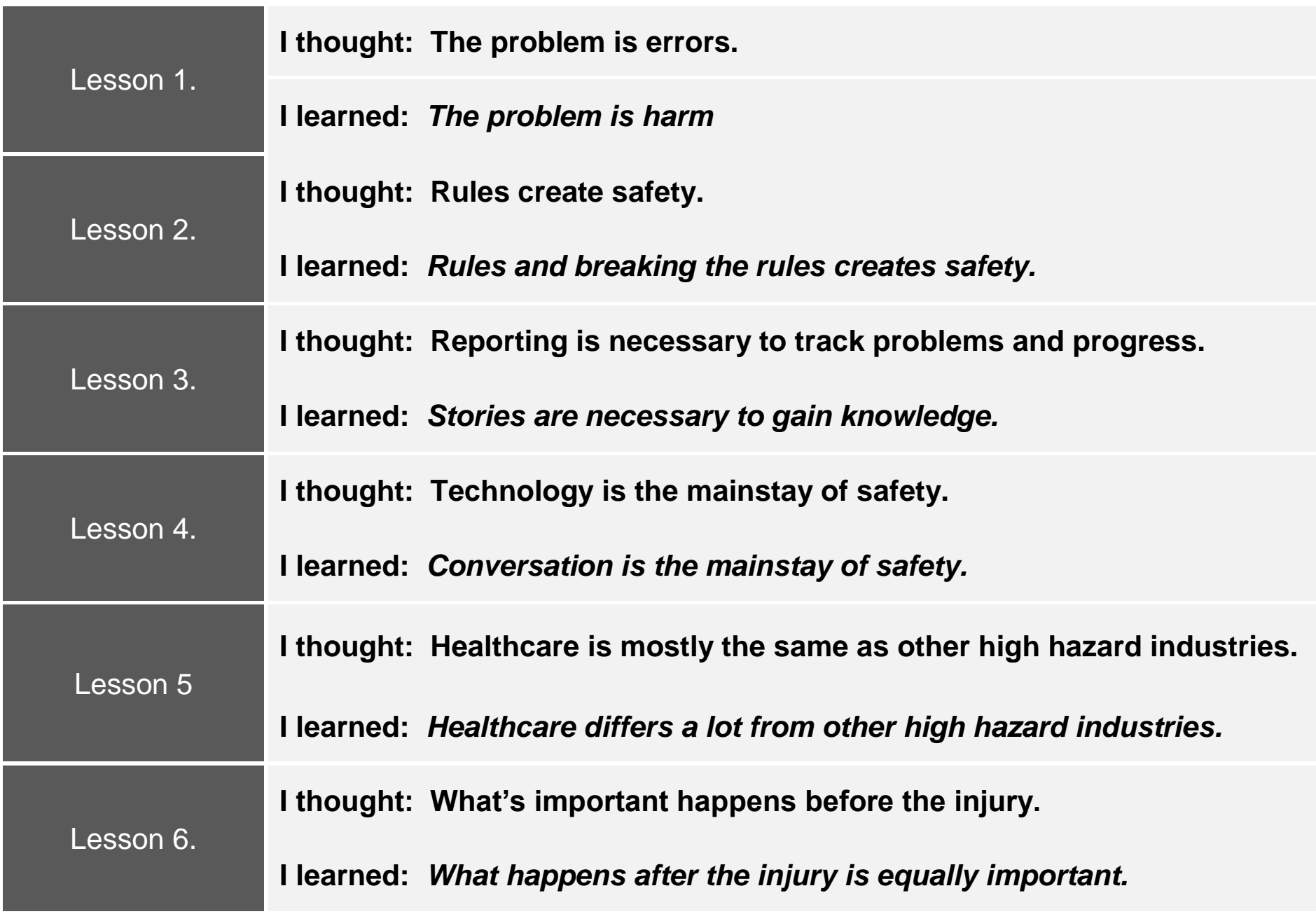

Lessons from a novice are copied from a 2002 article by Sir Don Berwick, a widelyregarded safety scientist. Berwick's 'lessons' are relevant to the research question which proposes that multiple views of safe patient care exist. Furthermore, Berwick states that his perception of patient safety altered with critical appraisal, thus illuminating the importance of ongoing research regarding the phenomenon of patient safety. 


\section{Setting the Scene}

\subsection{Introduction}

Harm directly caused by healthcare is experienced by 1 in 10 patients in the developed world (World Health Organisation (WHO), 2014). Indeed, this has led to the WHO stating that patient safety is an endemic concern. With regulators, governments, healthcare professionals and the public calling for safer care and reduced patient harm, the scale of preventable harm that continues to occur in health care is surprising. In the last decade, several 'watershed' reports have detailed preventable deaths, and examples of unsafe patient care continue to feature in international news items.

\subsection{The global context}

The magnitude of harm experienced by patients has been well documented since 1999 through a number of watershed papers. The most widely quoted is 'To Err is Human' from the United States (US). This paper compared the number of patient deaths in US hospitals to a jumbo jet full of passengers crashing every single day (Institute of Medicine [IOM], 2000). Following this US report, global attention has focussed on three major public enquiries about health care safety in the United Kingdom (UK). Seddon (2012) suggests the UK National Health Service (NHS) most closely resembles the NZ healthcare system. Therefore, an understanding of patient safety in the UK is considered to be of particular importance for this thesis. In 2001, the enquiry into Bristol Royal Infirmary identified avoidable deaths of 29 children following cardiac surgery (Department of Health [DH], 2001). This was followed by the Shipman enquiry, which investigated the murder of approximately 250 patients, by a general practitioner over a 20-year period ( $\mathrm{DH}, 2007)$. The most recent enquiry into deaths at Mid Staffordshire hospital is perhaps the most significant. The resulting report estimates 400 to 1,200 patients died as a result of poor care over four years (DH, 2013). All of the enquiries evoked a series of similarly worded responses from the government at the time. The UK DH committed to learn from causes, act on recommendations, and prioritise patient safety in healthcare. 
Patients must be kept safe within an increasingly interdependent and complex system of healthcare provision across the globe. More than thirteen thousand different diseases, injuries and syndromes are recognised, and most are particular and difficult to treat (Gawande, 2009). Increasingly complex patient comorbidity is accompanied by a progressively elaborate socio-technical environment, which further strains the healthcare system (Hollnagel, Wears and Braithwaite 2015). Providing safe care to the acutely ill is increasingly difficult to balance with operational demands in busy acute care environments, such as emergency departments (Ardagh, 2009). Expectations of individual patients, the wider community, and regulators must be carefully considered against patient need, available resources, and engaging with and caring for staff. My cumulative experience leads me to believe patient safety in acute care has unique challenges. These are predominantly created by patient variability, the increasing complexity in technology and treatment options or time-critical interventions and the dynamic state of operations. The view that patient safety in acute care is unique is supported by Hollnagel et al (2015) who argue:

"Systems such as intensive or emergency care cannot be decomposed in a meaningful way and the functions are not bimodal, neither in detail nor for the system as a whole. On the contrary, everyday work is - and must be - variable and flexible" (p. 3).

Interest in the phenomenon of 'patient safety' has intensified and the WHO has identified 13 areas for 'patient safety action' over the last decade. National programs have focused on these areas, such as 'Matching Michigan' in the UK (Bion et al., 2012), and 'Safer Surgery' in NZ (Health Quality and Safety Commission [HQSC], 2015a). Evidence suggests front line healthcare professionals consider current approaches are limited, wrong or misplaced. For example, a recent survey supported by the American Nurses Association found only $57 \%$ of nurses in the US, UK and China believe patient safety programs are effective (General Electric, 2011). Also concerning is that $34 \%$ of participants in a national survey of NZ healthcare professionals would not feel completely comfortable having a family member treated in their hospital, unless they were present to monitor care (Martin, Mason, Lovelock, Cumming and Hider, 2015). Acknowledging the response rate of the NZ survey limits interpretation (estimated 10\%), it could alone signal a lack of engagement with the 
current approach, especially as a significant majority of participants had or were currently involved in quality improvement initiatives previously in their DHB.

This year the WHO prioritised the phenomenon at a 'global action summit', where the Director General announced a global movement for patient safety to:

"Galvanize international policy and governmental actions to prioritise patient safety at all levels." (WHO website, 2016a)

Healthcare systems are challenged to provide safe care in a fiscal environment, complicated by the global recession and spiralling costs. Poor care costs money as a result of legal action taken against health care systems, and the health burden created by patient harm. The financial burden may explain the recent increase in the global attention on safer care provision. Indeed, Baker (2012) suggests the 'improvement' agenda has emerged to help reduce these costs by focusing on value and eliminating waste. 'To Err is Human' estimated the cost of harm on a national scale in the US in 2000 . This was a compelling amount; $\$ 37.6$ billion to $\$ 50$ billion for adverse events, $\$ 17$ billion to $\$ 29$ billion US dollars for preventable adverse events (IOM, 2000). In 2002, the cost of harm in NZ was estimated as $\$ 1.6$ billion NZ/1.16 US billion dollars (Brown et al., 2002), equating to $1.75 \%$ of NZ gross domestic product (GDP). The trend continues, and in 2014 the WHO estimated $20-40 \%$ of healthcare spending is wasted because of poor quality care.

Patient-centred care is at the heart of the improvement agenda for quality and safety and recognises the contribution of patients and families to safer care provision. Patient-centred care is:

"providing care that is respectful of and responsive to individual patient preferences, needs, and values and ensuring that patient values guide all clinical decisions." (IOM 2001. p. 40).

Patients and their families contribute to safety in a number of ways including: helping reach an accurate diagnosis; deciding about appropriate treatment; ensuring treatment is appropriately administered, monitored and adhered to; and identifying adverse events and taking appropriate action (Vincent and Coulter, 2002). Patient centred care can also present clinicians with increasingly complex dilemmas about 
whether care is safe or unsafe - for example, if a patient insists on treatment the clinician considers harmful due to its futility and likely side effects.

\subsection{The New Zealand (NZ) context}

NZ, the setting for this study, has a public secondary and private primary healthcare system. The Ministry of Health (MH) sets strategy and policy development and district health boards (DHBs) are responsible for the day-to-day healthcare operations. DHBs plan, manage, provide and purchase health services for the population of a geographical area $(\mathrm{MH}, 2016)$. Reducing harm continues to be a strategic priority in NZ, with the $\mathrm{MH}$ stating:

"The health system minimises harm to people, by ensuring that it honestly and openly tracks harm when it occurs, and learns from mistakes, so that the system as a whole can improve." (MH, 2016a p. 20)

Although this is a recent statement NZ has strived for a safer healthcare system since the Cartwright enquiry in 1988. The enquiry related to a 1966 study that monitored women with major cervical abnormalities without definitive treatment, and without their knowledge or consent, and findings contributed to sweeping changes in law and practice around health consumers' rights (Else, 2010). Patient safety became regulated through legal requirements and crown entities (bodies established by law in which the government has a controlling interest). In 1994, the office of the Health and Disability Commissioner (HDC) was established. The role of the HDC is to uphold a code of ten rights, which became law in NZ in 1996 (HDC, 2016a). The code sets out the rights and responsibilities of healthcare providers, such as the right to services of an appropriate standard, and the sanctions that can be imposed on those who breach the code. This unique model enables patients to request an investigation of any practitioner or provider they believe has harmed them. The HDC website describes their role as:

"A unique attempt to balance two diverse aims - the right of individuals to resolve disputes to their own satisfaction, and the right of the public to safe services and an accountable professional body" (HDC 2016). 
NZ regulatory reform has not stopped patients from experiencing harm. A retrospective review in 2002 found $12.9 \%$ of hospital admissions were associated with an adverse event, with approximately $2 \%$ of such incidents resulting in disability or death (Davis, Lay-Yee and Briant, 2002). In a recent study, using trigger tool methodology ${ }^{1}$, harm from adverse drug events was associated with nearly $30 \%$ of NZ hospital admissions (Seddon et al., 2012). International studies have suggested a significant number of patient safety incidents, like those found in the NZ studies, are preventable (IOM, 2000; MH, 2001; NPSA, 2008; and CEU 2009). The estimated rate of preventable events is significant ranging from $35 \%$ in NZ (MH, 2001) to $50 \%$ in the UK (NPSA, 2008). In NZ, like other countries, this has led to the establishment of a national body and in 2010 the Health Quality and Safety Commission (HQSC) was made legally responsible for a number of objectives relating to patient safety in the NZ Public Health \& Disability Amendment Act. The HQSC is a crown entity, its role is described by the board chair Professor Alan Merry as:

"Assisting providers across the whole health and disability sector - private and public - to improve service safety and quality and therefore outcomes for all who use these services in New Zealand" (HQSC website, 2015b).

The HQSC leads quality improvement programs and measurement activity to achieve this aim. The commission also has a role in improving understanding of the incidence of patient harm in NZ, and DHBs voluntarily report incidents to the HQSC for analysis, summarised in an annual national report.

\subsection{Emerging Approaches to Patient Safety}

Over the last decade approaches to patient safety in health have focused on systems used in high reliability organisations (HROs). High reliability theory (HRT) was developed in the US in the 1990s and emerged to challenge normal accident theory (NAT) which proposes accidents in tightly coupled (highly dependent), interactive systems are inevitable (Perrow, 1984). The original HRO researchers wanted to capture observed commonalities of operations among aircraft carriers, air traffic control, commercial aviation and nuclear power. Their intention was to

\footnotetext{
${ }^{1}$ Trigger tool methodology uses the global trigger tool (GTT) to identify adverse events, measure the rate over time and generate assumptions about the overall level of harm in an organisation (Griffin and Resar, 2009)
} 
demonstrate high risk, high hazard organisations can function safely. One of the researchers, Kathleen Sutcliffe, describes the characteristics of a HRO as:

"First, they operate in unforgiving social and political environments. Second, their technologies are risky and present potential for error. Third, the scale of possible consequences from errors or mistakes precludes learning through experimentation. Finally, to avoid failures, these organisations use complex processes to manage complex technologies." (Sutcliffe 2011, p.133)

Modern healthcare systems have similar characteristics to the HROs Sutcliffe describes. For example, many healthcare systems are publicly funded, and patient deaths are debated in parliament (e.g. UK, NZ). Additionally, existing models of health care provision are often fragmented and incoherent (WHO, 2012), and opportunities for technological advancement are appearing faster than doctors can keep up (Gawande, 2009).

HROs have made significant gains in safety outcomes over the last twenty years, further explaining the attraction for healthcare leaders. For example, the rate of accidents in aviation is at its lowest since 1973 with a trend towards fewer accidents overall (Evershed, 2015). Therefore, the HRO paradigm has dominated international approaches to policy and patient safety over the last fifteen years, and many HRO systems have been implemented wholesale into healthcare during this time. Systems include incident reporting, measurement and root cause analysis of adverse events, and an increasing reliance on protocols. Implementation of HRO systems has been accompanied by unprecedented international spending, increasing government regulation, and an awareness of systems improvement (Bagnara, Parlangeli, and Tartaglia, 2010).

Gawande (2009), a physician and patient safety scientist, suggests this phase of healthcare system and process change has associated patient safety with 'rules' and forms, observed as protocols, guidelines and checklists. Researchers have identified incident reporting has associated patient safety systems with a culture of blame and paperwork (Kingston, Evans, Smith and Berry, 2004; Leistikow et al., 2016; and Waring, 2005). HRO theory is broader than a focus on measuring incidents, stretching to social and organisational underpinnings of system safety and accident causation or prevention (Sutcliffe, 2011). Healthcare leaders have more recently 
embraced this, acknowledging patient safety is influenced by human factors, and problems with organisations and culture (e.g. Keogh, 2013).

Some authors suggest using HRT to analyse and explain adverse events in healthcare limits our understanding of what contributes to safe care. Hollnagel et al. (2015) suggest explanations of events where patients are harmed, cannot be limited to system performance, or explained by the linear models provided by either HRT or NAT. They suggest HRT has not developed in the same direction as health demand, complexity or strategy; stating safety thinking and safety practices have now reached an "impasse" (Hollnagel et al., 2015, p. 6) requiring a paradigm shift. Many authors agree change is required, with a focus on understanding sources of safety and resilience that already exist, an appreciation of the complexities unique to healthcare (Gawande, 2009; Hollnagel et al., 2015), and real time management of risk throughout the system (Vincent and Amalberti, 2016).

\subsection{Interest in the area of research}

I am a registered nurse, and have worked in acute care for sixteen years, in different clinical environments, in a number of roles and countries. A significant portion of my career has involved working with critically unwell patients, and leading clinical teams in this area. During this time, a number of changes to safety systems and quality improvement programmes have been introduced, such as early warning scores and critical care outreach teams in the UK. In my experience, the success of safety initiatives is dependent on organisational commitment, the buy-in of clinical staff, and a robust change process. My interest in patient safety as a research topic stems from an inability to understand the ongoing prevalence of preventable harm. Avoidable harm appears to be increasing in acute care environments, which does not make sense in the context of improved systems, processes, measurement and a political dialect that focusses on safety first. Furthermore, some of the most memorable experiences of my professional career relate to supporting patients and families who have experienced harm during an episode of care. Those that have most deeply affected me relate to supporting a significant number of healthcare staff harmed by their involvement in a patient safety incident, often feeling powerless to change the status quo. 
Quality is defined differently by healthcare professionals in NZ, and definitions are dependent on individual responsibilities and/or perspective (Seddon, 2006).

Professional experiences have led me to consider if the same is true of patient safety in acute care environments. My observation is that the majority of healthcare professionals embrace the concept of patient safety, but the phenomenon holds unique meaning to individuals and professional groups. I have been exposed to different cultures within acute care units, departments, organisations and professional groups and have often wondered if this contributes to safety culture. This study will consider if a deeper understanding of how safe patient care is interpreted in acute care environments, through a cultural lens, can provide new insights. The thesis considers if healthcare professionals have a similar understanding of the phenomenon of patient safety, using qualitative research methodology.

\subsection{Aims and objectives of the research}

This study will explore how patient safety is described from the perspective of clinical staff, and organisational management staff within acute care in a NZ context. The study objectives are to:

- Explore how intensive care and emergency care doctors and nurses, and senior organisational managers describe patient safety.

- Explore propositions identified from a literature review.

- Describe the factors perceived by intensive care and emergency care doctors and nurses, and senior organisational managers, as important in developing patient safety culture in an acute care hospital.

- Make recommendations to inform the development of patient safety culture for an acute care hospital setting in New Zealand. 


\subsection{Structure of the Thesis}

The thesis is presented over six chapters. Firstly, the literature review critically evaluates current definitions of patient safety. The review explores what is known about patient safety culture and its components, concluding with a purposeful research question which will add to the body of knowledge about the phenomenon. Chapter three describes case study design, the methodology and methods applied to understand the research question. The following chapter explores key findings, focussing analysis on the case and embedded case units. Chapter five is an in-depth exploration of themes generated by the research and contemporary literature, exploring the relevance to propositions generated in the literature review. The thesis concludes with central findings and recommendations to enhance our understanding of the phenomenon. 


\section{Literature Review}

\subsection{Introduction}

The literature review will critically evaluate current definitions of patient safety, and explore what is known about patient safety culture and its components. The chapter describes the contemporary understanding of patient safety in healthcare, and appraises the prevailing approaches. The chapter concludes by formulating a purposeful research question which will investigate identified gaps in understanding.

\subsection{Search Method}

A literature search was conducted in primary databases including the Cumulative Index of Nursing and Allied Health Literature, Medline, Pub Med and Blackwell Synergy. Secondary sources searched include the Cochrane database, TRIP and Google Scholar. The search extended to research and development publications from developed countries with similar healthcare systems to NZ, including the US, UK and Australia. Key search terms 'patient safety' and 'culture' or 'climate' were used, as well as 'safety culture or climate' 'organisational culture' or 'organisational climate' and 'evaluation'. Further search terms were used in conjunction with $\mathrm{MeSH}$ searching. As patient safety is a new science, the search was not limited to acute care settings at this stage.

It is widely recognised that the modern patient safety movement commenced with the publication of 'To Err is Human' in the US in 2000 (Leape, 2015). To account for this the literature review was limited to the date range 1999 to 2015. Boolean operators were used to increase sensitivity and specificity, particularly when searching Pub Med. Increased sensitivity generated too many opinion papers and review articles, so the limit 'research' was applied in Pub Med. Increased specificity generated the most current useful results. Following initial review and identification of emerging themes a secondary search was performed. This used the terms 'physician perceptions,' 'nurse perceptions' and 'managers' perceptions AND/OF 'patient safety.' This search yielded additional results, including qualitative papers. 
Grey literature sources were searched including patient safety-related government websites. The inclusion of grey literature is important as patient safety is an emerging area, and as such is developing its evidence base. A number of media articles and websites were identified through a local context search in Google NZ using the same terms. NZ government websites and online dictionaries were searched to provide definitions.

\subsection{Inclusion and exclusion criteria}

The primary aim of included evidence is to define, explain, understand or measure the phenomenon of patient safety culture. Sources include qualitative and quantitative empirical studies, books and grey literature. This thesis seeks to understand the significance of international bodies', governments' and consumers' interest in patient safety culture. The strength and prevalence of opinions may have some influence on culture, and therefore grey literature is relevant.

Empirical research from across the globe, including developing countries, has been included as socio-economic, population and cultural differences may influence patient safety in different countries. This allows the literature review to explore if differences in or between countries - with their own unique culture - might influence the phenomenon. Empirical research was excluded if it addressed risk management, quality improvement or safety, without any linkage to culture.

\subsection{Results}

Key search terms 'patient safety' AND 'culture' AND 'evaluation' yielded the most results. A total of 22 studies met the criteria; 19 quantitative and 3 qualitative papers. The primary focus of current research is the measurement of patient safety culture using survey methodology. This might be explained by the WHO stipulation that:

"The first step toward reducing patient harm is to understand its magnitude" (Mauro, 2016 p. 1133).

This statement ensures patient safety research focuses on the relationship between safety culture and patient safety outcomes (Kitch, 2008).

The majority of survey tools for measuring safety culture were developed in the US (e.g. Makary, Sexton et al., 2006; Sexton 2005). Surveys vary considerably in the 
dimensions measured, psychometrics, general characteristics and their use in research (Colla, et al., 2005). Seven components of patient safety culture are recognised in different survey tools. These are leadership, evidence base, teamwork, communication, learning, and a just and patient centred approach (Samner, Lykens, Singh, Mains and Lackan, 2010). Studies have compared surveys, and note variation in the validity of their testing. (Colla, Bracken, Kinney and Weeks, 2005; Makary, Mukherjee et al., 2007; Robb and Seddon, 2010). The key psychometric measure for most is Cronbach's alpha, and none of the surveys have been validated against in-depth interviews (Singla, Kitch, Weissman and Campbell, 2006). The most commonly used and widely validated survey tool is the Hospital Survey on Patient Safety Culture (HSOPSC). The HSOPSC used in the majority of papers in this review and has a dataset of over 600 US hospitals for comparison, which may explain the preference for this tool in the literature.

Papers measuring safety culture were purposefully chosen from a number of countries, as differences within countries provide a rich source of cultural data. Countries include Taiwan, Turkey, the Netherlands, Saudi Arabia, the US and NZ. Safety culture survey tools have been used to measure baseline safety culture, and raise the profile of safety culture (Robb and Seddon, 2010; Sexton, 2011). Research has attempted to understand if safety culture is associated with patient outcomes (Huang et al., 2010; Smitts et al., 2012), and test the usability of survey tools (Wallis and Dovey, 2011). Research has measured safety culture using a variety of tools in acute and primary care settings in hospitals (Pronovost et al., 2003; Robb and Seddon, 2010; Smits et al., 2012), cities (Alahmadi, 2010; Bodur and Filz, 2009; Wallis and Dovey, 2011) and more widely within countries (Chen and Hung-Hui, 2010; Hellings, Schrooten, Klazinga and Vleugels, 2007; Singer et al 2003). One study included examined differences in a number of components of safety culture across three countries (Wagner et al., 2013). Survey tools have more recently appeared in national quality improvement programmes where they have been used to evaluate the impact quality improvement interventions on safety culture $(A H R Q$, 2013; HQSC, 2015a; Sexton, 2011).

Two research papers use qualitative methods to explore cultural barriers to patient safety initiatives, and one utilised a survey tool to explore safety culture in focus groups. The number of non- empirical papers, books, national guidelines and 
professional sites relating to patient safety culture is vast. To manage this inclusion was limited to acute care environments at this stage of the research process.

\subsection{Defining harm}

In 2009, the WHO recognised the significance of harm caused to patients by investing in an international classification system for patient safety (Runciman et al., 2009). The system recognises morbidity or mortality caused by patient harm using the same approach applied to cardiac disease or cancer. Classification is used to define, harmonise and group concepts, so global system improvement can occur. The WHO (2009) international terms of classification defines the phenomenon of patient safety as:

"The reduction of risk of unnecessary harm associated with healthcare to an acceptable minimum." (p.22)

To 'do no harm' is a founding ethical principle of many healthcare professions (National Ethics Advisory Committee, 2013). This is evident in the WHO definition, where safe care is defined by the absence, or minimisation of its opposite - harm. In healthcare organisations, and the literature 'harm', 'adverse events' and 'injuries' are often used interchangeably (Parry, Cline and Goldman, 2012). Consideration will be given to the difference between these concepts to provide an understanding of how harm is interpreted, measured and managed in the healthcare setting.

'Harm' and 'injuries' are usually explained as an outcome that negatively affects a patient's health and/or quality of life, as seen in these definitions from the online medical dictionary (2009):

"Harm is anything that impairs or adversely affects the safety of patients in clinical care, drug therapy, research investigations, or public health. Harms include adverse drug reactions, side effects of treatments, and other undesirable consequences of health care products and services."

"An injury is harm or hurt; usually applied to damage inflicted on the body by an external force, called also trauma and wound"

'Adverse events' are usually occurrences of harm classified according to the severity of the outcome for the patient, with death as the most serious outcome. There is a 
mandatory requirement to report adverse events in many countries, but in NZ the system is voluntary. In NZ, adverse events are defined as:

"One which has led to significant additional treatment, is life threatening or has led to an unexpected death or major loss of function." (HQSC website, 2015d)

\subsection{The measurement of harm}

The measurement of harm is limited by many factors, including the lack of an existing taxonomy and framework and different definitions (Parry et al., 2012). Approaches generally focus on measuring either specific types of harm, or 'all cause' harm (Parry et al., 2012). Measurement of 'all cause' harm is less prevalent and is achieved through a retrospective analysis of patient's notes. A specific methodology, such as global trigger tool (GTT), is employed to achieve this. In the US, another approach is to look for trends in administrative data, analysing pre-determined hospital codes associated with episodes of harm.

Most developed countries measure specific harm using 'incident reporting' systems to record, investigate and audit adverse events (WHO 2009). Hollnagel (2014) calls this approach 'Safety l', where safety is measured as "inversely related to the number of adverse outcomes" (p.94). In Safety I harm is reported after it occurs, and pre-set patient safety indicators, usually agreed at a national level, provide a classification framework, such as falls, medication errors and wrong site surgery. National agencies analyse, interpret and report data in most developed countries (the US Institute of Healthcare Improvement IHI, the UK National Patient Safety Agency NPSA and the Australian Patient Safety Foundation, APSF). 'Safety l' assumes the system is safe if there are no adverse outcomes. Common sense suggests this is not a reasonable assumption; especially as national bodies maintain an emphasis on increased reporting as an indicator of good safety culture (WHO, 2016a; Agency for Healthcare Research and Quality (AHRQ), 2014; DOH, 2015; and HQSC, 2015c, HQSC 2015d).

The possibility that healthcare is unsafe despite increased reporting rates is demonstrated in NZ incident reporting data. Harm is formally measured by counting the number of adverse events voluntarily reported to the HQSC each year, and there is evidence that harm is significantly underreported. The occurrence of adverse 
events in NZ is approximately $12.9 \%$ of all hospital admissions, with $2 \%$ resulting in disability or death (Davis et al., 2002). In 2009/10, there were 1.1 million hospital discharges in $\mathrm{NZ}(\mathrm{MH}, 2012)$. This means that in the same timeframe approximately $22,000(2 \%)$ serious and sentinel advents should have been reported to the HQSC. The actual figure was 374 (HQSC 2010), 17\% of the expected amount. A number of reasons could explain differences in harm formally reported, and harm experienced in NZ, including the existence of multiple pathways to report harm (ACC, HDC and office of the coroner). Alternative explanations relate to occupational and organisational culture and their relationship with current systems, which will be explored further.

Under-reporting of adverse events to the HQSC may reflect organisational concerns about reputational harm. Mid Staffordshire hospital has become synonymous with healthcare scandal, and is used as a cautionary tale in publications from many countries including NZ. The hospital has in fact renamed itself to escape the organisational reputation caused by its past. In NZ, adverse events are recorded in DHB incident systems, and for the last ten years have been published annually on the HQSC website. This approach is congruent with a key principle of patient safety science, namely that sharing information both between organisations, and with the public, is of vital importance (Leape et al., 2002). The importance placed on this practice is also evident in WHO strategy. The WHO is currently testing a minimum dataset for incident reporting, intending to record and share information across the European Union (WHO, 2016b). It is therefore surprising NZ DHB managers suggest the current approach to share and 'learn' from harm, in a national arena, is of limited value.

Hardy's (2013) qualitative study of managers in 20 NZ DHBs found that although participants support incident reporting to help them understand local risks, they questioned the value of sharing information formally on a national platform. Participants stated they would prefer to share information informally; such as at national quality meetings, or between DHBs by email. This leads me to conclude that organisational managers in NZ have concerns about reputational harm, as informal methods are less transparent to the public. Findings also explain underreporting rates at the DHB level. Organisational managers in Hardy's (2013) study claimed they downgrade adverse events to near misses. One manager states this is because 
clinical staff are "not able to look at it as objectively", (Hardy 2013, p. 52). This suggests definitions of what is harmful to patients, and who is harmed may differ between organisational and clinical staff.

The prevalence of the current approach to measure and target interventions to reduce harm is surprising given the evidence that healthcare professionals, especially doctors, do not embrace it. Incident reporting has been instituted in healthcare systems in many countries for some time, but the literature tells us that clinical staff grossly under-report adverse events (Allan and Judith, 2005; Sari et al., 2007; HQSC, 2015d). A US study concluded patients report many serious and preventable events not documented in medical records (Weissmann et al., 2008). Similar conclusions were found in a retrospective record review in the Netherlands. This study identified only $3.6 \%$ of events in patient records could also be found in formal reporting systems (Christians-Dinglehoff et al., 2011).

In NZ reporting of harm has increased by $40 \%$ since 2006 . DHB media releases often attribute increases in reporting to 'culture change', as an assurance that targeted interventions are working and 'safety culture' is improving. In press statement accompanying the HQSCs annual adverse events report 2014-2015 the HQSC board chair stated:

"The rise in the number of events reported reflects the culture change taking place in health care, with greater emphasis on learning from systems failings." (Merry, cited HQSC, 2015d)

The suggestion that safety culture is linked to incident reporting rates is congruent with Westrum's (1993) proposition. Westrum theorises a positive safety culture requires a 'culture of conscious enquiry', where individuals and groups within an organisation observe, enquire and make conclusions about safety known. Merry's (2015) statement appears to be underpinned by Westrum's thinking, proposing safety culture can be measured empirically in NZ, and that incident reporting rates are a reliable method. This is surprising as little agreement exists over a precise definition of culture in organisations, how it should be observed or measured, or how different methodologies can be used to inform both routine operations and/or organisational change (Scott, Mannion, Davies, and Marshall, 2003). 
The HQSC (2015) statement suggests healthcare leaders believe healthcare professionals are increasingly supporting incident reporting systems as important for patient safety. This view is incongruent with international research findings that the level of harm in healthcare is considerably higher than reported (ChristiansDinglehoff et al., 2011;Weissmann et al., 2008; Sari, Sheldon, Cracknell and Turnbull, 2007), studies that have identified doctors do not support incident reporting systems (Evans et al., 2006; Hardy, 2013, Kingston et al., 2004) and papers suggesting the value and purpose of incident reporting systems are under increasing international debate (Leistikow, Mulder, Vesseur and Robben, 2016). Furthermore, Brennan et al. (1991) conclude the number of adverse events reported does not equate to poor quality care, or necessarily indicate good care.

Merry's (2015) statement proposing increasing reporting rates indicates an improvement in safety culture was released in December 2015 and is incongruent with findings from a HQSC commissioned research study published in October of the same year. The study aimed to assess the quality and safety cultures in NZ DHBs, and explore the extent to which health professionals were aware of the Commission's work, using survey methodology. The majority of respondents (55$59 \%$ ) stated the HQSC is neither 'effective nor not effective', $30 \%$ 'effective or very effective' and $13-14 \%$ responded the current approach is 'ineffective' (Martin et al., 2015).

The results combined with the low response rate (10\%), require cautious interpretation. However, the findings suggest the current approaches are not tackling unsafe care, as defined by front line healthcare professionals in NZ. This is particularly surprising as a large proportion of respondents were, or had been, actively engaged in DHB quality and safety programs. The study concludes current strategies to measure and manage harm have limited effectiveness and support. These findings further support that there is a limited evidence base to claim increased reporting reflects engagement with incident reporting and improved safety culture in NZ. Indeed, increases in reporting rates may reflect increasing frustration with unsafe care, system function, or increasing harm. An alternative explanation is the professional culture of nurses (55\% of respondents), is more supportive or aligned with current NZ approaches, than medicine (11\% respondents). 
Braithwaite, Westbrook, Travaglia and Hughes (2010) claim that a heightened awareness of the risks associated with procedures may explain why doctors are less likely to report an adverse event than nursing staff. The perceived inevitability of 'necessary harm', and culture of 'in house' review of incidents (Kingston et al., 2004) may explain low physician response rates in survey research into safety culture in a number of studies (Provonost et al., 2003; Robb and Seddon, 2010; Singer et al., 2003; and Wagner, Smits, Sorra and Huang, 2013; Smits et al., 2012). Patient safety incidents are often normalised within medical culture as an inevitable part of work (Gawande, 2009; Pronovost and Colantuoni, 2009).

Alternative explanations are that physicians are not engaged with the patient safety movement in its current form or that organisational strategies aimed at improving safety are not congruent with doctors perceived needs or professional culture (Braithwaite, Westbrook, Travaglia and Hughes, 2010) Indeed, some authors suggest the culture of the medical profession has a different interpretation of what the 'just culture' or 'learning culture' essential for patient safety looks like, compared with managers or nurses (Kingston et al., 2004; Waring, 2005; Bosk, 2005).

\subsection{The patient perspective}

Gawande (2014) proposes patients can best decide what is harmful, and this will be defined differently by individuals. He suggests if healthcare professionals fail to account for what is meaningful for an individual patient, an unintended yet harmful experience can ensue:

"whatever we can offer, our interventions, and the risks and sacrifices they entail, are justified only if they serve the larger aims of a person's life. When we forget that, the suffering we inflict can be barbaric." (Gawande, 2014 p.10)

Gawande (2014) identifies that patients may describe safe care differently to healthcare professionals, suggesting incident reporting is not a reliable method to measure harm as experienced by the patient. This is supported by a recent systematic review that concludes:

"Clinicians should resist side-lining patient experience as too subjective or moodoriented, divorced from the 'real' clinical work of measuring safety and effectiveness." (Doyle, Lennox and Bell, 2013 p. 1) 
In NZ, the HQSC has attempted to address this knowledge gap using survey methodology to measure 'patient experience' across four domains. Meaningful interpretation at a national level is not possible. The survey has a small sample (1627 patients invited by email) with a poor total response rate (highest Feb 2016 $29 \%)$. There is also difficulty in examining the response rate at a local level as there is considerable variability in the response rate across DHBs (HQSC, 2016a). The response rate suggests the current domains, measured over the last two years might not reflect what NZ patients define as good and bad care. This is concerning as the WHO definition does not specify who decides what harm is considered to be 'necessary'. Therefore, harm is internationally defined though a classification which may not reflect harm as experienced by the patient.

In NZ, the code of rights states that all patients have a legal right to be fully informed (HDC, 2016a). In addition, the practice of open disclosure when harm occurs is advocated by key NZ professional bodies (Medical Council NZ, 2010; Nursing Council NZ, 2012). However, there has been a small increase in the number of complaints patients reported to the HDC over the last ten years (18\%), implying healthcare professionals may not identify every episode of harm that patients experience. The number of complaints referred by the HDC to the director of proceedings, who considers prosecuting cases where the law has been broken, has increased significantly in the same time period (82\%). This suggests harm that meets the threshold for a breach of consumer rights is increasingly identified and reported by patients, rather than organisations or professional staff.

\subsection{The Emergence of Patient Safety Science}

Patient safety science has emerged in response to the prevalence of 'avoidable harm' on an international scale. Patient safety science focuses on risk reduction via system interventions, and is described by key authors as:

"A discipline in the health care sector that applies safety science methods toward the goal of achieving a trustworthy system of health care delivery. Patient safety is also an attribute of health care systems; it minimizes the incidence and impact of, and maximizes recovery from, adverse events." (Emmanuel et al., 2008) 
Science is traditionally associated with prestige, and stems from the success of generating knowledge of the empirical world through recognised methods (Edwards, 2001). Defining patient safety as a science implies a preference to seek interventions based upon 'what is known', rather than what is merely believed about the phenomenon. Thus, patient safety has become a distinct subject worthy of study, using methods that are familiar and credible, particularly to doctors. Key authors have cemented the prestige of patient safety as a science stating it has:

"The potential to revolutionize health care, perhaps as radically as molecular biology once dramatically increased the therapeutic power in medicine." (Emmanuel et al., 2008)

The definition of the phenomenon as a science explains the implementation of strategies from industries with foundations in applied science. HROs usually have roots in engineering, and patient safety scientists have been heavily influenced by risk reduction approaches in these industries. Patient safety scientists and quality improvement specialists have adopted a number of systems from HROs into healthcare over the past fifteen years, especially from aviation and car manufacturing. The predominant strategy adopted into healthcare is root cause analysis (RCA).

In HROs significant gains have been made by leading researchers from various disciplines collaborating, and working independently, to analyse and diagnose tragic or near tragic events. The determination of the underlying causes of adverse events generates knowledge that may be used to prevent a similar recurrence (Reason, 1990). This appeared in US healthcare in the late 1970s where it was used to review "mishaps" in anaesthesiology (Cooper, Newbower, Long and McPeek, 1978 p. 399). The formal implementation of RCA into healthcare occurred after the publication of 'To Err is Human' in the US, when RCA became an accreditation requirement of the US Joint Commission. NZ followed some years later and in 2012 a HQSC National Reportable Events Policy identified RCA as the preferred method for adverse event review.

Emmanuel et al. (2008) suggest leaders of HROs view the current level of adverse events in healthcare as so high, that many of them would consider the health industry as existing in a state of 'chaos'. The implementation of structures and 
systems from HROs, such as counting, analysing and learning from adverse events, are certainly not providing the same results in healthcare (Hollnagel et al., 2015).

This may be because a central message of HRO's is that the key to safety does not lie in systems and processes alone. The pivotal contributor to safety is a culture which detects and corrects threats to safety in real time (Sutcliffe, 2011). In HROs risk management is also characterised by attention to weak signals, the identification of a problem and a strong response.

The fact that HRT is more than measurement and retrospective analysis of harmful events appears to have been forgotten by healthcare leaders. This may be because this is counter-intuitive to how humans normally behave, as we are susceptible to expectation bias. Information is incorrectly processed so we can reduce uncertainty and maintain a sense of control (Weick and Sutcliffe, 2001). A true HRO approach is described by the original theorists as mindful (Weick and Sutcliffe, 2001). Mindfulness is underlined by five principles that enable HROs to achieve reliability and deal with the unexpected. Three deal with anticipation (preoccupation with failure, reluctance to simplify, and sensitivity to operations) and two with containment (commitment to resilience and deference to expertise).

\subsection{Defining safety culture}

Culture is a multi-layered concept, originally derived from the anthropological context. Culture is, as its theoretical roots suggest, more complex than systems that deal with risks and hazards. This is because culture is created and maintained by people (Hofstede, Hofstede and Minkov, 2010; Schein, 2004). There are three fundamental concepts traditionally applied to safety culture research: normative, anthropological and pragmatist (Edwards et al., 2013). The normative concept describes the normative differences or characteristics that are absent or present in an individual or group.

Anthropology focusses on shared perceptions and values, believing culture is and ought to be owned by everyone (Haukelid, 2008). In the pragmatist concept, social order is about tangible practices; in healthcare, this might be expressed though the routine handling of artefacts, and power relationships. 
These concepts can be easily understood in Schein's (2004) model of organisational culture, which has three layers:

- visible (artefacts)

- espoused beliefs and values (which may appear through surveys)

- unconscious, invisible beliefs and values (that are taken for granted).

Definitions of safety culture are derived primarily from HROs, who recognise the phenomena's importance and complexity. James Reason summarises this well stating:

"Few phrases occur more frequently in discussions about hazardous technologies than safety culture. Few things are so sought after, and yet so little understood" (Reason 1997, p. 191).

The most widely used definition of culture in the literature is derived from corporate culture in the 1980s. In this definition culture is:

"the way we do things round here" (Deal and Kennedy, 1982, p.4).

There is debate as to whether safety culture is a subset of organisational culture (Richter and Koch, 2004), or a phenomenon in its own right (Reason and Hobbs, 2003). There is general agreement that safety is 'everyone's responsibility', requiring every individual to commit to an organisation's safety culture. The importance of the contribution of the individual is recognised in the most well-known formal definition of safety culture. This definition originated from the field of engineering following the 1988 Chernobyl disaster:

"That assembly of characteristics and attitudes in organizations and individuals which establishes that, as an overriding priority, nuclear plant safety issues receive the attention warranted by their significance." (International Nuclear Safety Advisory Group (INSAG), 1991 p.14).

The literature is complicated by confusing and inconsistent definitions over the use of the terms 'safety climate' and 'safety culture' which are used interchangeably. The difference between these two concepts is defined by UK Health and Safety Executive (HSE) as: 
"The term safety culture can be used to refer to the behavioural aspects (i.e. 'what people do'), and the situational aspects of the company (i.e. 'what the organisation has'). The term safety climate should be used to refer to psychological characteristics of employees (i.e. 'how people feel'), corresponding to the values, attitudes, and perceptions of employees with regard to safety within an organisation." (HSE, 2005 p. iv)

The HSE definition does not appear to prioritise the anthropological view of culture. This holds that shared values and attitudes are part of culture. Geertz (1973), a renowned anthropologist, acknowledges cultural context is seldom static. Certainly, in the healthcare literature 'safety climate' appears to refer individual's values and beliefs about culture within the context of a specific point in time. On the other hand, safety culture more commonly refers more to an aspirational ideal that can be generated, measured and controlled. In the healthcare context safety culture is usually referred to as patient safety culture. In their concept analysis Feng, et al. (2008) state the most widely influential definition of the phenomenon is:

"The product of individual and group values, attitude, competencies and patterns of behaviour that determine the commitment to, and the style and proficiency of, an organisation's health and safety programmes." (HSE, 1993).

\subsection{Significance of patient safety culture in the healthcare setting}

Harm can be attributed to poor patient safety culture, rather than a focus entirely on systems and processes. This has led to growing recognition of the necessity to transform organisational culture in healthcare (IOM, 2000, 2001, 2004; Hemman, 2002; Sutcliffe, 2011). Poor safety culture in healthcare organisations is identified as a major cause of harm in the watershed reports referred to in this thesis. The NHS report 'A Promise to Learn a Commitment to Act: Improving the Safety of Patients in England' (2013) reported findings from the Mid Staffordshire Hospital enquiry. It states:

"In the end culture will trump rules, standards and control strategies every single time.... achieving a safer system will depend far more on major cultural change than a new regulatory regime" (National Advisory Group on the Safety of Patients in England, p.10). 
The finding is relevant in the NZ context. The Health and Disability Commissioner Anthony Hill suggests there are on-going cases of patient harm caused by failure to speak up, and poor organisational culture. He states this is:

"The enduring theme of every international inquiry for the last quarter century, including the Cartwright enquiry" (HDC, 2013, p. 3).

Awareness of the significant relationship between safety culture and patient harm is gaining momentum. Many authors suggest safety culture is something that should be 'developed' alongside traditional processes and reporting systems in healthcare organisations (Hardy, 2013; Leape et al., 2009; National Patient Safety Foundation, 2015). The phenomenon is primarily studied within the management paradigm, perhaps due to perceived importance of the relationship between safety culture and organisational culture. Current research focus is on measurement of culture and the success of safety management strategies, inferring safety culture is as easy to churn out as a protocol about a new system of medication administration (Edwards, Davey and Armstrong, 2013). There is a limited knowledge base concentrating on the anthropologist worldview which holds culture can hardly be managed (Geertz, 1973). Indeed, Schein (2004) a key author on organisational culture suggests culture is so difficult to alter, managers should consider other options first when attempting change.

\subsection{The multiple perspectives of patient safety culture}

Normative approaches focus on the 'development' or 'creation' of patient safety culture, maintaining culture is and ought to be owned by groups. The goal of normative culture is described by Arnold (1983) as to overcome barbarity, whilst actualising higher goods, such as intellectualisation and deference to authority (Johnson, 2013). Culture is therefore used as an alternative control instrument to other forms of control in organisations, like bureaucracy (Haukelid, 2008).

The preference for the normative concept in the management paradigm can be explained by Haukelid's proposal; safety culture is viewed as a scale of measurement to establish the effectiveness of organisational policies and procedures (Edwards et al., 2013). This is evident in the most prolific model of safety culture in the healthcare literature. Westrum and Adamski's (1999) theoretical model 
of generative culture has normative underpinnings. Westrum and Adamski (1999) propose safety culture evolves along a line, through three stages.

The linear model begins with a pathological culture (caring less about safety than about not being caught); through calculative (blindly following all the logically necessary steps), to generative (in which safe behaviour is fully integrated into everything the organisation does). Westrum and Adamski's model requires measurement, and suggests interventions will generate and control a cultural ideal. The model is limited by its linear approach, assuming every individual and/or group (subculture) within an organisation is on the same point, and is or can be directed and controlled by the organisation. This view is incongruent with key theoretical work on culture. The phenomenon is viewed as an entity that cannot be controlled (Geertz, 1973), and organisational culture is described as a complex sum of cultural layers or subcultures (Schein, 2004).

The recognition of the importance of power relationships and their influence on healthcare culture has led to patient safety scientists using system interventions as 'forcing functions' for cultural change. A forcing function is a term often used in engineering to describe a behaviour-shaping constraint. The increasing popularity of the use of forcing functions suggests safety culture is viewed as a tool or solution that can be 'applied' to an organisation, and that this is achieved by the creation and maintenance of improvement interventions. Rowley and Waring (2011) caution against this approach, as it assumes culture is an entity or variable which can be managed directed and controlled.

Haukelid (2008) concludes current approaches ignore the challenges presented by cultural layers created by values and beliefs, adding that managers should be "more modest in their efforts to manage cultures" (p. 413). However, some studies have demonstrated forcing functions, such as checklists, improve safety culture in theatres and intensive care, using survey research methods (Gawande, 2009; Pronovost, 2003; Sexton et al., 2011). These interventions have had an influence on culture by enabling staff to raise concerns, and flattening traditional hierarchies. Interestingly they have been developed and led by clinical staff including psychologists and doctors. An increasing use of forcing functions intended to alter culture may explain why increases in incident reporting have been attributed to culture change. They also 
explain the attraction of measuring safety culture to demonstrate the 'effect' of the intervention.

Anthropology views culture as the sum of shared perceptions and values, and has been compared in a society to memory for an individual, or "designs for living that have proven effective in the past" (Triandis, 1989, p. 511). In simple terms a number of factors ensure the same actions are repeated by the cultural group (Brinkman, 2007). Anthropological theoretical approaches to patient safety culture include those that seek to understand language, social structures, aesthetic patterns, history and location. In safety culture research the concept usually incorporates cross-cultural psychology, predicting behaviour and understanding differences in behaviours between individuals and groups (Edwards et al., 2013).

Rowley and Waring (2011) write from an anthropological perspective, suggesting patient safety culture is influenced by deeply-held, complex systems of shared and tacit meanings, acquired through years of socialisation. They propose a good safety culture requires shared attitudes, practices and beliefs about patient safety. This proposition is supported by Weick and Sutcliffe's (2001) descriptions of safety on aircraft carriers in the US Navy. Flight carriers have an exceptional safety record, despite complex and difficult conditions, and the crew work long hours over a period of several months. The resulting safety record could be explained by an enhanced shared understanding of safety culture, created by the physical location of the ship and the presence of an uninterrupted dominant culture (The US Navy).

The importance of shared attitudes and beliefs is further supported by Leape and Berwick (2005). They suggest changing even just a few policies and procedures requires all personnel to share one vision, and be personally responsible for safety. Findings from two qualitative studies suggest a shared vision for patient safety does not currently exist in the healthcare context. Both studies conclude clinical staff will work outside of organisational structures if they feel that they are not relevant to an individual patient, or their professional practice (Waring, 2005; Dixon-Woods et al., 2009). 
The proposal that safety culture relates to, and is positively or negatively influenced by complex systems of shared and tacit meanings, is important in the healthcare context. This is because the cultural context is distinctly different to those of traditional HROs such as flight carriers, or nuclear power.

"Healthcare systems are composed of numerous professional groups, departments, and specialties with intricate, nonlinear interactions between them; the complexity of such systems is often unparalleled as a result of constraints relating to different disease areas, multidirectional goals, and multidisciplinary staff. Within large organizations such as healthcare systems, the numerous groups with associated subcultures might support or be in conflict with each other." (AI-Sawai 2013, p. 285).

Keesing (1987) agrees culture is not a homogeneous whole. Indeed, in healthcare, conflicting subcultures with different history, language and behaviours may influence 'the product of individual and group values, attitude, competencies and patterns of behaviour' the HSE (1993) cite in their definition of safety culture. Furthermore, the 'product' (patient safety) is on a continuum and is dependent on the context. This infers patient safety culture can be poor or exceptional at any one point in time, and is dependent on the actors in play. Rowley and Waring (2011) appear to write from this perspective and suggest current systems, with their approach to a 'taxonomy of error' fail to acknowledge the importance of this variation.

A number of studies found safety culture components between units of different specialities within one hospital varied (Hartmann et al., 2008; Singer et al., 2009; Smits, Wagner, Spreeuwenberg, Van der Wal and Groenewegen, 2009; Zohar, Livne, Tenne-Gazit, Admi and Donchin, 2007). The variation could be explained by the fact that definitions of the components of safety culture arises from fields that do not always comfortably coexist. Fields include quality improvement, healthcare management, medicine, nursing, engineering and other industries (Feng, Bobay and Weiss, 2008).

In the anthropological worldview, it is appropriate to consider the influence of occupational culture on safety culture. In survey research, occupational culture might explain high scores for teamwork within units (Alahmadi, 2010; Bodur and Filzs, 2009; Chen and Hung-Hui, 2010; Robb and Seddon, 2010; Wagner 2013), and lower scores in teamwork across units, and in transitions or handoffs (Alahmadi, 2010; El 
Jardarli, 2010; Robb and Seddon, 2010; Wagner 2013). Every organisation has its own unique culture, defined as the set of deeply embedded, self-reinforcing behaviours, beliefs, and mindset that determine 'the way we do things around here' (Shein, 2004). Organisational culture has occupational subcultures, created within departments (such as an ED or ICU) and occupational groups (such as doctors and nurses). Occupational culture is:

"A distinctive pattern of thought and actions shared by members of the same profession and showed in their language, morals, outlooks, beliefs, and traditions." (Psychology Dictionary Online,2016).

Occupational culture is created from both an organisational frame of reference, and the meaning the work holds for an individual or group (Van Maan and Barley, 1982). For example, an ED can create a unique culture which is a sum of underlying beliefs, traditions, and values that go beyond what is written down in organisational values or job descriptions (Person, Spiva and Hart, 2012). Bourdieu (1998) suggests certain bodies of knowledge are reproduced through training and professional habitus, which in turn influence organisational culture. It is therefore surprising that occupational culture has been ignored in approaches to improve patient safety culture, though many authors agree this is a particular challenge in medicine (Bosk, 2005).

The fact that differences in occupational culture may influence safety culture is evident in the survey research. Response rates are high for nurses (mean 49.5. Median 50), and low for physicians despite targeted strategies (Mean 12.2, Median 8.3). This may indicate that (1) safety culture is more important to nurses; (2) safety culture is described in a way that resonates with nurses but not doctors in current surveys; or (3) current safety culture strategies better meet nurses perceived needs. This is supported by qualitative research, for example Waring (2005) found a divergent occupational responsibility in critical incident reporting, with doctors suggesting this belonged to nursing because:

"the culture of nursing is familiar with form filling... and more amenable to management control" (Waring, 2005 p. 1933). 
Doctors in this study suggested that reporting incidents made no difference to patient safety, viewing it as an attempt to colonise the professional culture of medicine through:

"extension of managerialism, and an erosion of professional status" (Waring, 2005, p. 1934).

Poor response rates may reflect the pattern of resistant behaviour evident in medical culture. Medical culture has largely resisted attempted incursions into what it views as its scope of practice, and has been rewarded by largely retaining its power base (Allsop, 2006; Bourgeault and Mulvale, 2006; Boyce, 2006). It may be that medical culture views 'patient safety' as synonymous with 'clinical risk', and is therefore owned by medicine. This may explain findings in a study where a checklist and bundle for central lines was introduced in ICUs by Sexton et al. (2011). Two dimensions: 'feedback and communication about error' $(p<0.01)$ and 'teamwork within unit' improved $(p<0.01)$. Interestingly, although communication about error increased, the non-punitive response to error decreased. This may be because feedback or challenges about doctors' practice by professions outside of medicine was negatively perceived, disrupting the usual 'way we do things round here'. Conversely, the increase in teamwork could be explained by healthcare professionals outside of medicine feeling empowered to speak up, and therefore completing the survey.

Findings from several studies using survey methodology suggest attitudes and beliefs about patient safety are not shared across management and clinical groups. A definite discrepancy in the attitudes and experiences of senior managers and those of non-managers was a finding in several studies using survey methodology (Alahmadi, 2010; Pronovost, 2003 and Singer et al., 2009; Wagner et al., 2013). A study in the US using the SAQ survey found staff perceived that supervisors had a greater commitment to patient safety than the senior leaders (Pronovost et al., 2003). Likewise, more than half of the participants in Alahmadi's (2010) study in Saudi Arabia stated managers overlook safety problems that reoccur, with $43 \%$ indicating patient safety issues existed within their units. 
An ethnographic study of acute wards in four hospitals in the UK sheds light onto the survey findings. Dixon-Woods et al. (2009) used interviews and observation to understand how clinical staff and organisational managers view risk. The researchers concluded the classification and response to risk is influenced by the role and professional accountabilities of different healthcare professionals. This evidence suggests efforts intended to improve safety are unlikely to have an impact if they fail to account for the impact of occupational culture.

\subsection{Conclusion}

Culture is often compared to an iceberg (Hall, 1976). When we initially look at an iceberg, we think that 'what we see is what we get'. The same is true with patient safety culture; we falsely assume we grasp a particular people group because of what we see, when in reality nine tenths lies 'below the water'. The literature tells us that culture is strongly linked to values, suggesting the use of the positivist paradigm alone limits the depth of our understanding. The paradigm focuses on establishing objective scientific facts, through scientific method (Broom and Willis, 2007), and views the truth as absolute, distinguishing facts from values (Dickson Swift, James and Liamputton, 2008).

The heavy use of a survey approach limits our understanding to espoused beliefs and values, reflecting only one layer of culture. The survey approach assumes we already have a good understanding of what lies beneath the surface, yet components have not been validated using qualitative methods in the healthcare setting. Guldenmund (2007) proposes questionnaires are not a reliable method for exposing the core of organisational safety culture, evaluating only safety management. This is an important view as survey components of safety culture have been developed in the corporate world, by studying what has worked in HROs. This narrows our understanding of the phenomenon in healthcare by applying untested assumptions about the underlying culture of health, viewing it as essentially the same.

Differences in safety culture found in survey research between countries suggest there may be components created by cultural context that are poorly understood. Safety culture is not as positive in countries using HSOPS compared against the US database including NZ survey (Robb and Seddon, 2010). The difference could be 
explained by the development of survey components in the US, which has a different culture to NZ. American culture emphasises self-reliance and individualism (Ludwick and Silva, 2000). In NZ individualism exists, but culture is also influenced by the Māori worldview, emphasising the wellbeing of the group, rather than the focus on the individual (Berghan 2007). In addition, the US healthcare system is predominantly private, like traditional HROs, whereas in NZ secondary healthcare is provided in a public system.

The literature tells us safety culture is vulnerable, and can be enhanced or derailed by individuals and groups (Alahmadi, 2010; Dixon-Woods et al., 2009; Pronovost et al., 2003; Waring, 2005). The influence of professional culture is poorly understood, but study findings point to its importance. Poor response rates, particularly from doctors, suggest surveys' construct validity may be limited, and basic assumptions about safety culture in different parts of an organisation may be wrong. This is particularly important for this thesis as some authors suggest that occupational groups in non-clinical areas perceive a more positive safety culture than workers in high-hazard units, such as the emergency department (ED), intensive care units (ICU) and operating room (OR) (Hartmann et al., 2008, Singer et al., 2009). In addition, a thematic analysis of adverse events relating to patient deterioration in NZ found communication failure as the leading cause of harm (HQSC, 2015). This supports the proposition that acutely unwell patients present unique challenges, and that their safety is influenced by the cultural context. My own experiences led me to believe that safety culture is especially vulnerable when teams continuously reform to meet individual patients' needs, such as in EDs, or within medical emergency teams (MET).

The prevailing approach has not produced similar safety improvements in the healthcare setting as in HROs. This suggests the time has come to rethink how safety in healthcare is understood and that the relevance of HRT may be limited. Current understanding is limited for two reasons. Firstly, the description of a phenomenon is incomplete without a qualitative understanding of the subjective meanings of the social actors and their actions (Glasser and Moreno, 1989). Secondly, qualitative research can uncover observations about phenomena that demand the creation of new ideas and categories that might not emerge using a positivist approach (Ezzy, 2002; Strauss and Corbin, 1998). 
A qualitative approach is valuable, as the cost of implementing systems from HROs wholesale may be that we do not see, and investigate, those aspects that do not fit our presuppositions about the phenomenon. A qualitative approach will enhance our understanding adding to the body of knowledge on patient safety. This is essential, as improving our understanding may contribute to safer patient care, and explain differences in attitudes to current systems. This thesis will therefore use qualitative methodology and methods to answer the research question 'How do healthcare professionals describe patient safety?' 


\section{Study Design}

\subsection{Introduction}

This chapter will describe and present rationale for use of case study methodology to achieve the research aims. As chapter two has concluded, current understanding of patient safety is informed by a predominantly positivist approach. To add to the body of knowledge in this area, the phenomenon of patient safety will be explored by use of qualitative research method. In using this research approach, the complex social world within which patient safety is experienced will be understood, through the subjective descriptions of multiple healthcare staff in specific organisational settings: providing a case study focus. This chapter will detail selection and definition of the case, the embedded case units and the study participants. Exploration of the data collection and data analysis strategy then follows. A description of how methodological rigour and research ethics were attended to concludes the chapter.

\subsection{Research Paradigm}

The research question assumes multiple participant descriptions of the phenomenon exist, and can be uncovered. The dominant philosophy of this research is therefore grounded in the interpretative constructivist paradigm. A paradigm is an overarching philosophical or ideological stance, a system of beliefs about the nature of the world (Rubin and Rubin, 2012). In the research setting, this is the ideological perspective from which knowledge is produced.

Research philosophy is underpinned by epistemological and ontological beliefs. Epistemological beliefs relate to what constitutes valid knowledge, and how we obtain it, and ontological beliefs relate to what constitutes reality and how we understand existence. Constructivist researchers believe knowledge is gained by capturing the relationship between the subjects of study and their reality (Cresswell, 2014). Their ontological view is that knowledge is socially constructed, rather than 'set in stone' (Rubin and Rubin, 2012). Constructionist philosophy is appropriate for this study as it supports the generation of multiple participant meanings assuming there is no 'objective' truth, and individuals have multiple realities, explanations and descriptions of phenomena. 
Constructionists use research methods that achieve 'thick description'. 'Thick description' is a detailed account of field experiences in which the researcher uncovers the patterns of cultural and social relationships, and puts them in context. Renowned anthropologist and ethnographic researcher Clifford Geertz (1973) proposes culture can only be explained through "thick description" (p.9), which he states is of vital methodological importance. Therefore, constructionist research methods are relevant for this study, as it seeks to understand patient safety culture. Geertz (1973) proposes 'thick description' goes beyond a factual account of culture, suggesting hermeneutic interpretation is required. Gadamer (1976) suggests hermeneutic interpretation requires a circular relationship, where the researcher attempts to understand human beings in a social context and the movement of understanding "is constantly from the whole to the part and back to the whole". (p.117).

Taylor first suggested safety science should use a hermeneutic lens in 1981. Modern hermeneutics includes the analysis of verbal and nonverbal forms of communication, language and symbols and takes into account prior actions and aspects that affect communication (Little, 2008). Hermeneutic interpretation makes meaning of that information, drawing inferences and judging the match between explicit information and some abstract or implicit pattern (Gadamer, 2004). To put it simply, 'thick description' is the researcher's construction of other people's constructions of what they and others are up to. For the purposes of this research culture it is defined as how participants share and create definitions of patient safety, how they develop shared meanings of the phenomenon within their unique context, and how participants associate meaning with events or actions that relate to patient safety.

\subsection{Case Study Methodology}

Key authors in the field of case study design are Stake (2005) and Yin (2009). They propose case study design is particularly relevant when the aim of research is to describe and contextualise a phenomenon within a specific contemporary or cultural context. In case study research, multiple sources of data are collected, converging in one result - increasing our understanding of a phenomenon. The use of case study methods will add to the body of knowledge generated from survey research, as case studies can be both superior and complementary to surveys (Yin, 2009). 
Furthermore, surveys cannot investigate contextual conditions as they limit the variables used to investigate it. The study design in this thesis is more closely aligned with Stake's (1995) constructionist philosophical assumptions, than Yin's (2009) post positivist philosophy. However, both approaches are important, and I will attempt to replicate the emphasis Yin (2009) places on cohesion and consistency of design.

A case study is defined as:

"A bounded system." (Stake, 1995 p. 2)

"An empirical inquiry that investigates a contemporary phenomenon in depth and within its real-life context, especially when the boundaries between phenomenon and context is not clearly evident." (Yin, 2009 p. 18).

Case study research is especially relevant for this thesis as it is philosophically attuned to the critical incident technique (CIT) used by HROs. Flanagan (1954) describes CIT as:

"A procedure for gathering certain important facts concerning behaviour in defined situations." (p. 355).

This study is an instrumental case study, as it seeks to provide insight into the phenomenon of study and make generalisations. The case will be examined, scrutinised and detailed, but the case itself is of secondary interest, playing a supportive role to facilitate understanding of the phenomenon (Stake, 1995).

The identification of culture as having visible and invisible facets lends itself to a case study design. Case study research requires the use of multiple sources of data to achieve 'thick description' of a phenomenon and provide a hermeneutic lens to understand culture. Data sources included in this study are interviews and focus groups from multiple participants and field notes maintain a holistic view of the phenomenon. The use of multiple sources of data will provide an understanding of the context in which the phenomenon is situated, and the complexity of the setting. Complexity is defined as the presence of unfamiliar, unplanned and/or unexpected sequences of events within the context, which like culture, can be either not visible or not immediately comprehensible (Perrow,1984). 


\subsection{Propositional Generalisation}

The goal of case studies is to expand and generate theory, not to enumerate frequencies as in positivist research (Lincoln and Guba, 1985; Yin, 2009; Stake, 1995). Case studies generalise to other situations (on the basis of analytic claims), whereas surveys and other quantitative methods tend to generalise to populations (on the basis of statistical claims). Yin (2009) proposes that single case studies can form the basis of significant explanations and generalisations about a phenomenon.

Lincoln and Guba (1985) agree, suggesting one can evaluate the extent to which conclusions are transferable to other times, settings, situations and people if a phenomenon is described in sufficient detail. Stake (1995) calls this 'propositional generalisation'. In this thesis, embedded case study design will increase the level of detailed inquiry by providing 'sub units' for analysis. The use of embedded case study design accounts for assertions that multiple cultures can exist in organisations, often with co-existing or conflicting subcultures (Deal and Kennedy, 2000; Schein, 2004; Wallis and Dovey, 2011; Wagner et al., 2013). The use of sub units is relevant as this study seeks to understand the perspective of sub specialities within medical, nursing and managerial professions.

The use of case study design allows unique descriptors to be generated from language unique to a professional group (Stake, 1995). In this study, descriptors may offer explanations of how healthcare professionals describe patient safety within a specific context. This makes them meaningful to outsiders sharing the same professional culture. Development of unique descriptors may therefore support language which is more acceptable to certain healthcare groups, and can be generalised to acute care settings.

Both Yin (2009) and Stake (1995) suggest case studies benefit from prior development of theoretical propositions to guide data collection and analysis. Propositions are important as they form the foundations of the conceptual framework generated from findings (Miles and Huberman, 1994; Stake, 1995). Baxter and Jack (2008) suggest using propositions allows a researcher to place limits on the scope of the study, and increase the feasibility of completing the project. Propositions can be generated from a variety of sources including the literature, professional experience, theories and empirical data (Baxter and Jack, 2008; Stake, 1995; Yin, 2009). 
Propositions in this study were generated from the literature review, and my own experiences and informed development of the interview guide (Table 1).

Table 1: Propositions used in this study

\begin{tabular}{|c|c|}
\hline Proposition & Evidence \\
\hline $\begin{array}{l}\text { 1. The majority of healthcare } \\
\text { professionals embrace the concept of patient } \\
\text { safety, but the phenomenon is defined } \\
\text { differently by services, professions and } \\
\text { individuals. Safety culture is dependent on } \\
\text { the actors in play, including the patient. } \\
\text { Therefore, safety culture is vulnerable and } \\
\text { can be enhanced or derailed by individuals } \\
\text { and groups. There is a negative stigma } \\
\text { around harm and this may contribute to a } \\
\text { culture of safety in a positive or negative way. }\end{array}$ & $\begin{array}{l}\text { Alahmadi (2010), Al-Sawai (2013), } \\
\text { (Bodur and Filzs (2009), Bosk (2005), } \\
\text { Chen and Li (2010), Coster (2013) } \\
\text { Dixon-Woods et al. (2009), El Jardarli } \\
\text { et al. (2010), El Jardarli et al. (2014), } \\
\text { Gawande (2009), Hartmann et al. } \\
\text { (2008), Pronovost et al. (2003), Robb } \\
\text { and Seddon (2010), Rowley and } \\
\text { Waring (2011), Singer et al. (2009), } \\
\text { Smits et al (2009), UKHSE (2005), } \\
\text { Vincent (2006), Wagner et al. (2013), } \\
\text { Waring (2005), Weick and Sutcliffe } \\
\text { (2001), Zohar et al. (2007), DOH } \\
\text { (2013), Decker (2013), Flannagan } \\
\text { (1954), Hollnagel et al. (2015). }\end{array}$ \\
\hline $\begin{array}{l}\text { 2. Patient safety in acute care has unique } \\
\text { challenges. In these environments HRO } \\
\text { approaches focussing on rules and systems } \\
\text { alone may not be the best solution to improve } \\
\text { safety culture. Therefore, the measurement } \\
\text { of adverse incidents may not be a reliable } \\
\text { indicator of safety culture. }\end{array}$ & $\begin{array}{l}\text { Amalberti, Auroy, Berwick and Barach } \\
\text { (2005), Flannagan (1954), Gawande } \\
\text { (2009), Hollnagel et al. (2015), IHI } \\
\text { (2015), Smits et al (2012), Smits et al } \\
\text { (2012). Cook and Rasmussen (2005) }\end{array}$ \\
\hline $\begin{array}{l}\text { 3. Zero harm is unobtainable in healthcare } \\
\text { environments. Therefore, harm may need to } \\
\text { be redefined. There is a point where harm } \\
\text { reaches an acceptable level, and acceptable } \\
\text { harm may be defined differently by the } \\
\text { healthcare professionals involved. }\end{array}$ & $\begin{array}{l}\text { Bagnara et al. (2010), Gawande } \\
\text { (2009), Hollnagel et al. (2015), } \\
\text { Pronovost and Colantuoni (2009), } \\
\text { Vincent and Amalberti (2016), WMA } \\
\text { (2015), WHO (2009). }\end{array}$ \\
\hline
\end{tabular}




\subsection{The Case}

Ritchie and Lewis (2003) see the defining feature of a case study as being the multiplicity of perspectives which are rooted in a specific context. The authors are asserting the contextual conditions in which a case is situated are highly pertinent, and contribute to 'thick description'. A common mistake novice researchers make is to try and answer a question that is too broad, or a topic with too many objectives (Baxter and Jack, 2008). Authors (Miles \& Huberman, 1994; Stake, 1995) suggest placing boundaries on a case can prevent this, determining what a case is and is not, a technique they call 'binding'. Definitions will contextualise the healthcare setting, case and sub units of analysis, so multiple perspectives are uncovered within a defined scope. Definitions are provided from the NZ MH. This provides consistency and context, whilst acknowledging these are definitions from the furthest point from the patient.

The site acting as the case study for this research is a Tertiary Hospital (TH) in the NZ public secondary healthcare system. A tertiary hospital is:

"A Hospital that sees large numbers of similar cases from a wide catchment area (and) can offer more specialised staff and equipment more efficiently, and of a higher quality than small hospitals can." (MH, 1998 p.10).

The TH serves the health needs of a major NZ city, providing tertiary-level healthcare to a population of approximately a million across a large geographical area. A range of speciality services are available including paediatrics, neurosurgery, trauma, cardiothoracic and vascular. Care was provided by a predominantly clinical workforce, similar in make up to other NZ hospitals (approximately $40 \%$ nursing and midwifery, $14 \%$ medicine and $16 \%$ allied health).

\subsubsection{Sub units of analysis}

Stake (1995) surmises 'propositional generalisation' in a case study can be enhanced by the researcher's interpretations. He suggests this is because interpretation is seen through a researcher's own experience, leading to 'naturalistic generalisations'. The use of areas that are congruent with my clinical background is considered to be especially relevant. Providing methodological rigour is attended to, 
the use of familiar subject matter in a complex clinical environment enhances my ability to interpret the data as a novice researcher.

Sub units identified for analysis are two distinct clinical areas providing care to acutely unwell, primarily adult patients (figure 1). The units were the intensive care unit (ICU) and emergency department (ED). Additional subunits of analysis are the different professional groups working in, or responsible for these areas represented in the participant sample (figure 2). These are doctors, nurses and organisational managers. The units of analysis were categorised using Reason's (1997) terminology. The 'sharp end' denotes clinicians interacting with patients. Managers responsible for designing and enforcing the patient safety agenda within the organisation (and the wider healthcare system when this applies) were categorised as the 'blunt end' of the organisation.

Figure 1: Embedded Cases - Units of Analysis

\section{Units of Analysis Within the Case}

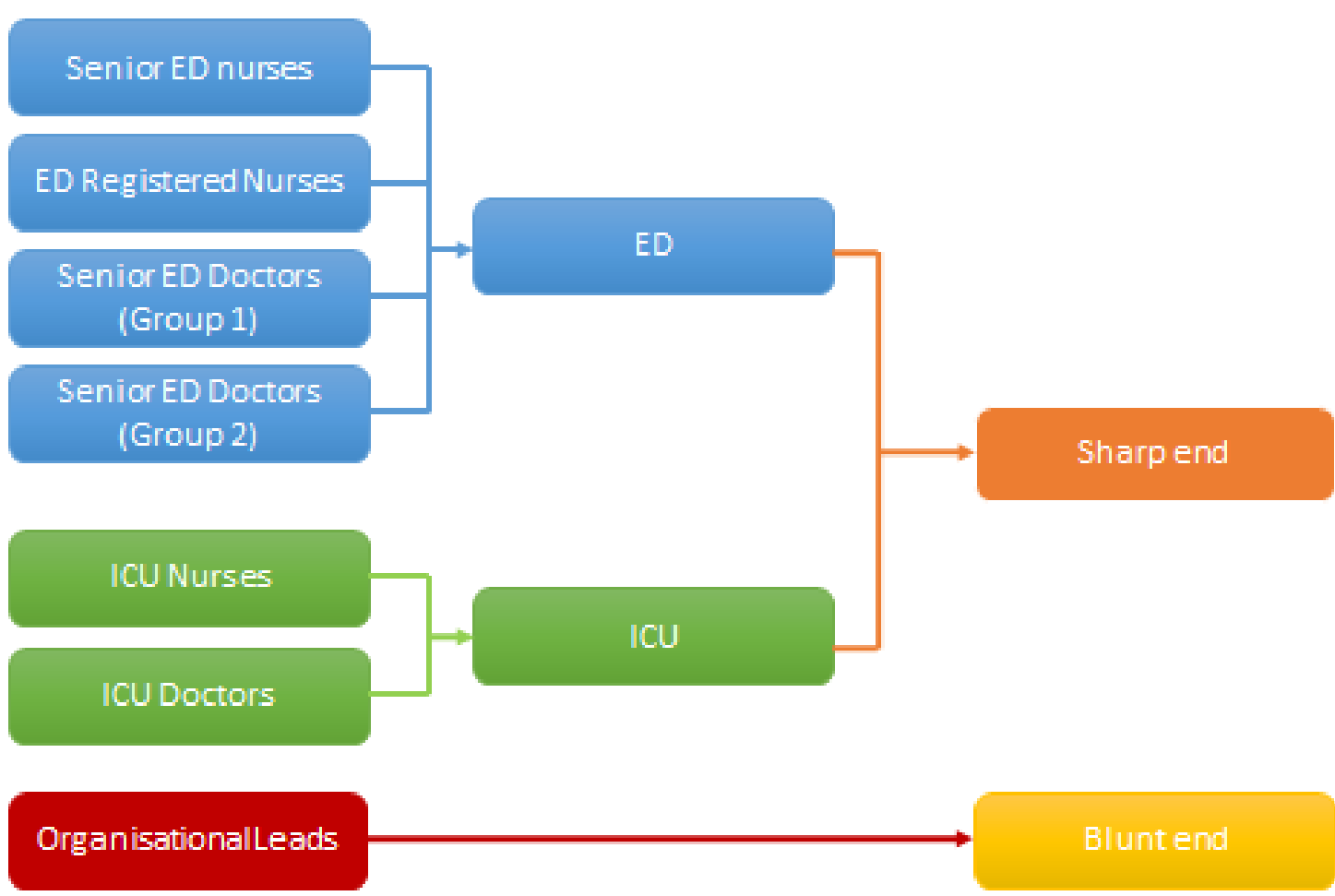


Figure 2: Embedded Cases - Sub Units of Analysis

\section{Sub Units of Analysis: Professional Group}
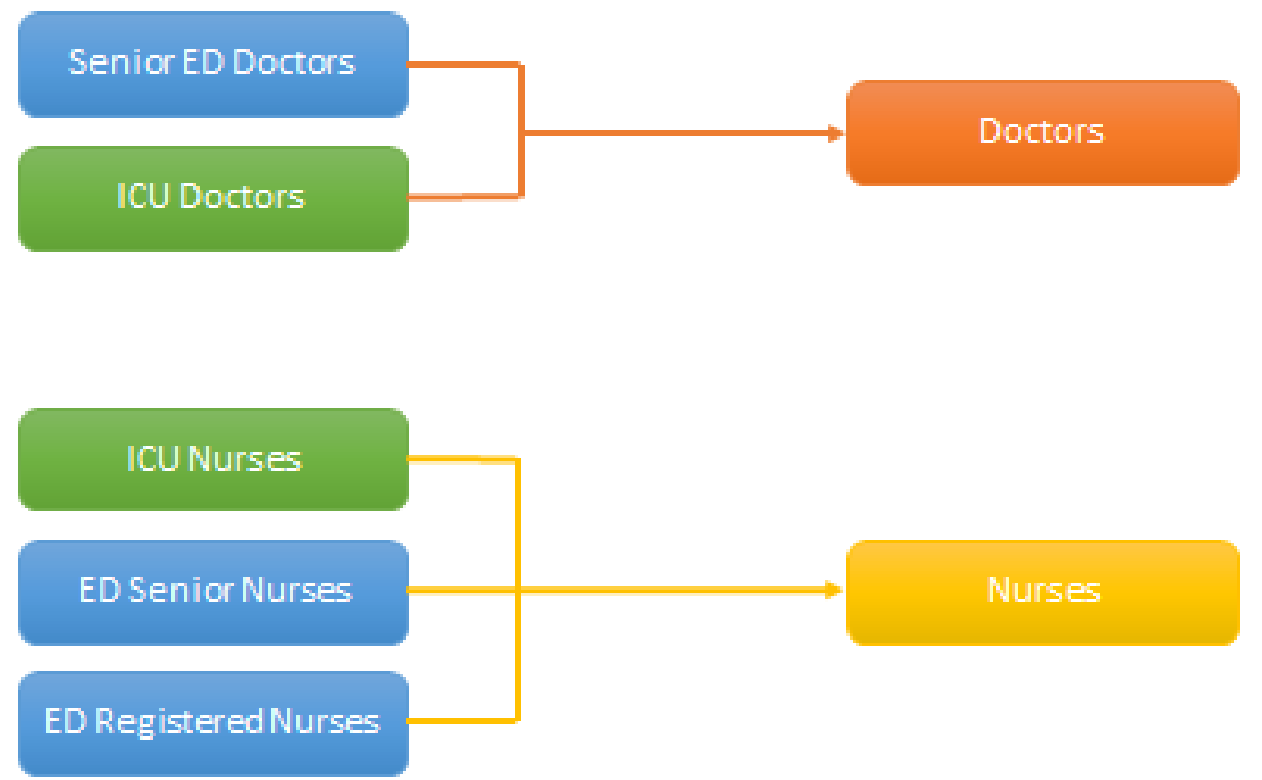

The units of analysis provide a rich source for detailed description. The ICU and ED both provide care for acutely unwell patients with varying severity of illness, and are required to continually reshape around patient needs. The opportunity to test the proposition that multiple subcultures and descriptions of the phenomenon exist is afforded by their similarities and differences.

An Intensive Care Unit (ICU) is defined as:

"An intensive care unit is a specially staffed and equipped, separate and selfcontained section of a hospital for the management of patients with lifethreatening or potentially life-threatening conditions. Such conditions should be compatible with recovery and have the potential for an acceptable future quality of life. An ICU provides special expertise and facilities for the support of vital functions, and utilises the skills of medical nursing and other staff experienced in the management of these problems" (JFICM, 1997; cited MH 2005 p. 7). 
An Emergency Department (ED) is defined as:

"An Emergency department is responsible for treating people who have a serious illness or injury that requires urgent attention" (MH NZ website 2016b).

\subsection{Gaining access to the field}

A DHB was approached and provided locality approval in a six-week timeframe. The process required signed authorisation to be submitted to the research department from clinical leads in ICU and ED, and an executive sponsor. Doody, Slevin and Taggert (2013) suggest a gatekeeper is identified by the researcher to act as a liaison between the participants and the researcher. Gatekeepers identified were supportive of the research aims, a strategy which intended to maximise participant numbers. Permission was sought from clinical leaders (doctors and nurses) of ED and ICU by phone or in person with follow up in writing. Clinical leaders were provided with participant information packs and consent forms. The documents were distributed beforehand by email, and clinical staff were asked to consider participating in the research during an existing meeting. Permission was sought from organisational managers in writing. This was followed up a phone call with the appropriate executive assistant, and the process was supported by an executive lead acting as a gatekeeper to the blunt end.

\subsubsection{Participants}

Case study research uses a purposeful sampling strategy, as participants are chosen based on their unique experiences, and what they can add to the phenomenon of study (Yin, 2009). The benefit of purposeful sampling is that:

"Any common patterns that emerge from great variation are of particular interest and value in capturing the core experience and central, shared dimensions of a setting or phenomenon" Patton (2015, module 33)

In this study participants were recruited from the sub units of 'professional groups'. This was limited to doctors, nurses and organisational managers permanently based in, or with management accountability for the ED and/or ICU. This strategy provides a deeper understanding of the 'subculture' of the specific groups. Recruitment of participants from organisational management maintains focus on the case in question, and provides context for analysis of the sub units of ED and ICU. 
Recruitment of doctors and nurses allows for data collection from the professional groups that make up the majority of the clinical workforce in these areas.

Organisational managers are defined as:

"One who handles, controls, or directs, especially a. One who directs a business or other enterprise b. One who controls resources and expenditures." (the freedictionary.com 2016)

Interviews are a commonly used research method in qualitative research and were used to gather data from participants. An interview is defined as:

"A conversation that has a structure and a purpose. It goes beyond the spontaneous exchange of views in everyday conversations, and becomes a careful questioning and listening approach with the purpose of obtaining thoroughly tested knowledge." (Kvale and Brinkman, 2009 p. 3).

Interviews are appropriate for this study as they produce knowledge through social action. Furthermore, the knowledge gained is harmonious with the philosophies of phenomenology and hermeneutics (Kvale and Brinkman, 2009; Rubin and Rubin 2012). Methods used in this thesis are focus groups and in-depth interviews.

\subsubsection{Data Collection Methods}

Multiple methods of data collection are typically used in case study design (Stake, 1995; Yin, 2009). This section will discuss and justify the sampling strategy and methods chosen for the study. Yin (2009) suggests the external validity of a case study report is increased when it is accompanied by a formal database. A case study database containing transcriptions of interviews, the case study guide and documents relating to data analysis will be retained for five years. Data security was attended to; for the duration of the research paper files were kept in a locked filing cabinet, and other data was held in a password-protected file.

\subsubsection{Focus Groups}

A focus group usually consists of a group of five to eight participants led by a researcher (Kruger and Casey, 2009). The aim of focus groups is to bring forth different perspectives of a phenomenon (Kvale and Brinkman, 2009; Rubin and Rubin, 2012). Focus groups increase sample diversity through lively interaction, with 
some authors stating this is what makes them superior to in-depth interviews (Rubin and Rubin, 2012; Then et al., 2014). Furthermore, thick description emerges from the rich data provided by group interaction within the structure of a focus group. The work of doctors and nurses is time pressured, predominantly occurring on the clinical 'shop floor', where the healthcare workforce is concentrated. Focus groups are recognised as practical and feasible to overcome this challenge and gain access to doctors and nurses (Plummer and D'Amato, 2008). Gatekeepers were identified and asked to provide opportunities during non-clinical time or unit meetings, to maximise participation. Gatekeepers are in positions of power over participants, therefore researchers must be cautious that participants could be coerced (McConnell and Henry et al., 2010). To attend to this the voluntary nature of participation was stated during marketing and at the beginning of every focus group. As authors suggest a conductive environment ensures focus group participants feel safe and in control (Doody, Slevin and Taggert., 2013), gatekeepers were asked to identify a room within the clinical area which would fulfil these criteria.

The strategy of homogenous segmentation separates participants into similar groups. Homogenous segmentation enables candid discussion, allows freedom of expression and limits peer pressure and the introduction of bias (McDermid, Peters, Jackson and Daly, 2014; Onwuegbuzie, Dickinson, Leech and Zoran 2009). Therefore, gatekeepers were asked to separate opportunities for data collection by professional group, initially identified as senior doctors, doctors, senior nurses and registered nurses. Group dynamics or pre-existing relationships can influence a participant's response; therefore, it is important to attempt to capture non-verbal data and ensure all participants have the opportunity to contribute (Rubin and Rubin, 2012). In focus group research, an observer is usually recruited to capture nonverbal data which will contribute to thick description and strengthen analysis, and a university supervisor was identified for this purpose. Observer information was captured within a predefined template. Using a university supervisor provided opportunities for formal debriefing, recognising the skill of interviewing is a "craft" that can be learnt through practice (Kvale and Brinkman, 2009, p. 17). Debriefing will increase rigour in this research as opportunities are provided to reflect on novice research skills and explore initial findings. 


\subsubsection{Qualitative interviews}

Qualitative interviews are preferred over focus groups when group interactions can be detrimental or highly sensitive (Dickson-Swift et al., 2008.) Disparity in social status, similar to those created in an organisational hierarchy, can prevent focus group members from talking freely to each other (Redmond and Curtis, 2009). Factors considered to be of potential importance to organisational managers include disparity in power relationships, and the influence of political positioning on data collection. In-depth interviews will prevent the introduction of these factors during the data collection process.

Sensitive research is described by Lee (1993 cited Dickson-Swift et al., 2008) as research that is perceived as threatening (where deviance is uncovered), intrusive, or political. The case is situated within a public healthcare system, led by the government, and as such is a political organisation. Organisational managers are closest to the political system, and deviance from standards uncovered in the research process could be viewed as threatening. Organisational ethnographers suggest this perceived threat can reduce participant candour (Ybema, Yanow, Wels and Kamsteeg, 2009). Interviews are a method used to lessen feelings of intrusion, whilst maximising opportunities for participants to disclose issues that they may ordinarily keep closed (Dickson- Swift et al., 2008). Interviews provide a safe environment for candid discussion with organisational managers, and minimise potential bias in data collection. On a practical level interviews provide the best opportunity to gain access to these participants as they can control the time and place.

\subsubsection{Interview Guide}

An interview guide (Appendix 1) was developed for both focus groups and interviews, containing general descriptive questions, and questions developed from propositions. Interviews can be structured, semi-structured or unstructured (Rubin and Rubin, 2012). A semi-structured approach was used, providing the flexibility to use a funnelling technique with participants and uncover 'thick description'.

Vaughn et al's. (1996) template supported the development of an interview guide. The guide was developed to help me as a novice, allowing questions on easier 
topics to be introduced first, followed by more sensitive topics. Morgan (1997) suggests this approach is essential in sensitive research as trust and rapport can develop. A pilot focus group $(n=4)$ of senior acute care nurses tested the appropriateness of questions, evaluated my technique, and supported development of the final template. A pilot provided an accepted approach to enhance rigour in focus group research, and provided the opportunity to reflect on my interview technique by listening to the audio recording (Rubin and Rubin 2012, Yin 2003).

Data from interviews was derived from individual participant responses to the following questions:

1. "I would like to ask you a really broad question; can you describe what patient safety looks like in your role/daily practice?"

2. "I would like to ask you a really broad question again. What organisational and clinical factors are required to develop a culture that supports patient safety in acute care?"

A probing technique was used to frame follow up questions, providing the opportunity to delve more deeply into participant experiences. Probing questions were generated from participant commentary and pre-set questions developed from the literature (Appendix 1). Responses were taped and transcribed for analysis. Many authors suggest extensive reliable data collection from interviews is dependent on the quality of the relationship between the interviewees and the researcher. Traits that develop rapport include warmth, enthusiasm, objectivity, having a nonthreatening nature and being caring (Krueger \& Casey, 2015; Rubin and Rubin, 2012). To establish initial credibility and trust I introduced myself focusing on my clinical background in focus groups, and my management and clinical background with managers. Throughout the interview process consideration was given to emulating the traits described by authors. Debriefing, supervision and listening to tapes of interviews provided opportunities to reflect on the quality of the relationship, helping me to learn and adjust my style throughout the data collection process. 


\subsection{Data Analysis}

A detailed description of the methods used for analysis enhances the rigour of a study, and allows for replication (Creswell, 2014; Stake, 1995; Yin, 2009). Therefore, the following section will describe the strategy used for data analysis. In research that uses case study design, data analysis uses theoretical propositions to guide analysis, following a general inductive approach.

A general inductive approach is:

'Preparing the data for analysis, conducting different analyses, moving deeper and deeper into understanding the data (some qualitative researchers like to think of this as peeling back the layers of an onion), representing the data, and making an interpretation of the larger meaning of the data.' (Cresswell, 2009, p. 183).

The data analysis process (figure 3) drew on several recognised qualitative analytic approaches widely quoted in the literature (Bryman, 2008; Miles and Huberman, 1994; and Pope, Ziebland and Mays, 2000). Data analysis is an iterative activity, and in Figure 3 the iterative process is described under the umbrella term 'horizontal pass' (Borkan, 1999). This describes movement back and forth across the data, asking questions from collection to analysis and back again. Strauss (1998) suggests researchers should ask the same specific set of questions, with a novice researcher believing both 'nothing' and 'everything'. In this thesis, propositions guided questions asked during analysis. 


\subsubsection{Data Analysis}

Figure 3: Data Analysis

\section{Data Analysis}
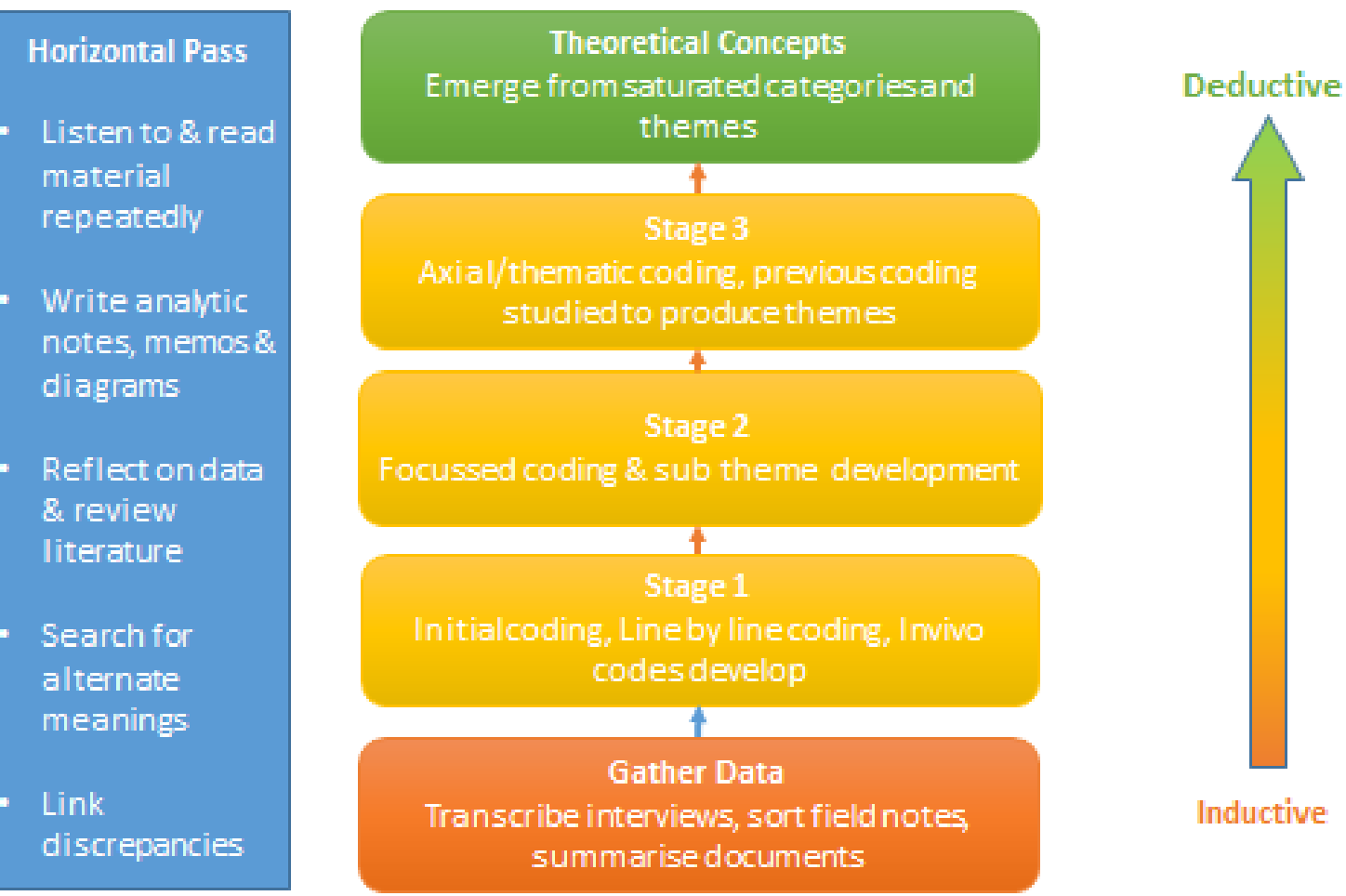

Inductive

\subsubsection{Coding}

Codes were developed in four stages, concluding in the generation of theoretical concepts once data saturation was met. Codes are:

"A word or short phase that symbolically assigns a summative, salient, essence capturing and/or evocative attribute for a portion of language based or visual data." (Saldana, 2009, p. 3).

The initial phase of coding looked for obvious codes, defined as basic units of text relating to the research question. Key words were highlighted, with notes made within each line of text. Holloway and Wheeler (2010) suggest this 'line by line' approach to coding minimises the risk of a novice researcher jumping to conclusions during the initial phase. The strategy aimed to develop 'in vivo codes' which are 
codes expressed in a participant's own language (Kruger and Casey, 2009). The inclusion of 'in vivo' codes is more likely to produce findings epistemologically in harmony with the language of health professionals, which is important in this thesis as language is an essential anthropological component of culture.

Many authors suggest the novice researcher should transcribe some if not all of the data collected to develop research skills, and add to the depth of analysis (Holloway and Wheeler, 2010; Kvale and Brinkman, 2009). As this was not possible and a professional transcriber was used due to time constraints, great care was taken to listen to recordings on multiple occasions. Recordings of interviews and focus groups were re-read whilst listening to transcripts over and over. Ayres et al (2003) advocate the use of 'over reading' alongside coding to uncover what is implicit in the text. Over reading ensures a rigorous approach to interpretation, helps make sense of comments and brings the data to life. To attend to this, observer notes and field notes were reviewed against text segments to evaluate potential hidden meanings and triangulate data. As codes were developed they were entered into an excel spreadsheet for interpretation, and a separate spreadsheet was developed for each unit of analysis.

As codes were identified, sub themes were identified from patterns in the data. Authors suggest this is when the research story emerges as codes begin to cluster together (Bryman, 2008; Salander 2009). Conceptual links were identified from words or sections of text that held the same implicit and explicit meaning, and sub themes that participants expressed as significant. Sub themes were entered onto the spreadsheet for each unit of analysis and were searched for contradictory points of view, and new insights. Interview text segments that conveyed the central essence of sub themes were linked where their meanings appeared to be similar.

Qualitative inquiry demands "meticulous attention to language and deep reflection on the emergent patterns and meanings of human experience" (Salander, 2009, p. 11). To uphold this principle, the coding cycle was repeated several times within each unit of analysis. Analysis of every unit was completed before interpretation was attempted. The final phase of coding is the development of theoretical concepts. These were developed as major themes were compared with each other. A literature search was conducted at this stage. This enhanced my understanding of the 
relationship between key themes from coding, general theory and empirical research aiding interpretation. A theoretical model was developed from analysis of the data in the context of the literature and the research setting.

Ayres et al (2003) suggest many qualitative researchers fail to go beyond theme identification, stating analysis is not complete until themes are reintegrated in manner which demonstrates how they work within the case. This step is required to provide a basis for idiographic generalisation. This means there is confidence in the findings about what is unique about the case, and allows the researcher make assumptions beyond it. Lincoln and Guba (1985) agree, suggesting one can evaluate the extent to which conclusions are transferable to other times, settings, situations, and people if a phenomenon is described in sufficient detail. To attend to this, themes and codes were analysed, contextualised and interpreted alongside a detailed description of the setting, the literature and the researcher's experience.

Throughout analysis I explored, defined and tested rival explanations both autonomously (listening to tapes, re reading text, exploring propositions) and through supervision (comparing analysis, exploring propositions and testing assumptions). Yin (2009) suggests confidence in the findings will be increased if propositions and rival propositions are addressed and accepted or rejected, as part of the iterative process. Propositions focused interpretation on the research question, and rival propositions generated from supervision, coding and the literature provided alternate explanations of the phenomenon. This was considered to be especially important where there were contrasting perspectives within and between the occupational groups.

Documentation at each stage of analysis ensured a rigorous process. Throughout the coding process, thoughts and ideas were documented on transcripts, or as comments within spreadsheets. These were new propositions to ask the data, follow up in the literature, or questions relating to my own professional experience. Documentation of the iterative process at each point in time was a purposeful strategy to reduce bias, and ensure ideas were not 'lost' in large volumes of data. A rigorous approach to coding is of vital importance, especially by a novice researcher. To attend to this requirement supervisors cross-checked initial and final coding against six transcripts from both interviews and focus groups across the sites. 
Coding and interpretation was discussed in supervision, including critical appraisal of findings. The movement of data from initial coding through to final themes is illustrated in the coding frame in Appendix 2. Furthermore, Appendix 3 and Appendix 4 provide examples of how the codes were developed.

\subsection{Ethical Considerations}

\section{Ethical research has a:}

"Responsibility to protect the privacy, safety, health, cultural sensitivities and welfare of human subjects." (Victoria University of Wellington, 2016.)

The majority of healthcare research uses the 1964 'Declaration of Helsinki' framework to achieve this (WMA, 2015). This framework is well suited to the positivist paradigm, but does not address the complexities presented by the constructionist paradigm. Multiple ethical issues can arise from using qualitative research methods that are more personal and have increased interaction and intrusion. Frameworks cannot fully account for the ethical impact of sensitive research, which may not be understood until research begins (Dickson Swift et al., 2008). Generally speaking, authors agree that qualitative research, and the intrusive nature of sensitive research, is lessened by broadly considering ethical principles (Dickson-Swift et al. 2008, Kvale and Brinkmann 2009). Ethical research is governed by five moral principles: respect for persons, honesty, benevolence, to do no harm, and justice. Several strategies uphold these principles in this thesis including informed consent, maintaining confidentiality, with special considerations for participant's wellbeing in sensitive research.

The Victoria University of Wellington Human Ethics Committee approved the research proposal (Appendix 5). Ethical approval required the development of participant information packs and consent forms (Appendix 6 and 7). These were emailed to participants by the relevant gatekeeper before interview, with extra copies available at every session. Participants were invited to discuss any concerns before written consent was obtained, and had the option to request feedback following completion of the thesis in the form of a summary document ${ }^{2}$.

\footnotetext{
${ }^{2}$ A copy of the thesis abstract was provided to the research participants either directly (blunt end) or via gatekeepers (sharp end)
} 
Stake (1995) suggests a researcher must leave an organisation without making anyone "less able to carry out their responsibilities" (p. 60). Participant vulnerability should be minimised in sensitive research, where researchers are bound by ethics of care, and the ramifications of participation must be considered (Dickson Swift et al., 2008). Patient safety has already been identified as a sensitive research topic, and has the potential to be threatening, or distressing to participants. Participants may have experienced harm as a patient, or moral distress as a second victim. Secondly, participants could reveal information when describing care that might be stigmatising or viewed as 'poor'; and finally, instances of patient harm reported during data collection have the potential to impact individual, organisational or political reputation. To attend to this a confidential employee assistance programme was offered to participants if they found any aspect of participation distressing. Participants were advised that gatekeepers were available for ongoing support or discussion. Supervision and observer debriefing were identified as appropriate forums to discuss any emerging concerns, so they could be respectfully managed.

All participants were asked to respect and maintain confidentiality of the data in focus groups and care has been taken to maintain the anonymity of the organisation in this thesis. Confidentiality was strictly adhered to, with agreements developed and signed by the observer and transcriber. In the results section, doctors and nurses are identified by their job title and a unique number e.g. EDRN1. There are a small number of organisational managers, with one person in each specific role. To maintain confidentiality, they are identified more generically, as $\mathrm{OM}$ and a number. A small number of participants in focus groups and interviews are known to me. When this has occurred, additional assurances were made including a commitment to accuracy, that findings would adequately represent experiences, and confidentiality would be maintained, as suggested by McDermid et al. (2014).

A follow up group will be offered to all participants to share findings on completion of the thesis. This will occur before the dissemination of research findings though publication and conference. This will provide an informal opportunity for member checking, enhancing rigour in the research, and maintain a respectful approach. 


\subsection{Ensuring methodological rigour}

Qualitative researchers traditionally usually use terms such as credibility, dependability and trustworthiness to describe a rigorous research process (Lincoln and Guba, 1985). This thesis will use the terms used by Yin (2009), and Stake (1995), which are reliability and validity. This maintains the focus on the authors who have guided the design of this research, whilst recognising these terms are more commonly associated with the positivist paradigm. Norris (1997) suggests that terms used in qualitative research are less important than "why we believe the things that we do, and how we justify the claims we make" (p.172).

Reliability relates to the ability of others to accurately replicate the research study in case study research, which Yin (2009) believes is of particular importance. In this study, this has been achieved through the development of key documents. This includes the case study protocol and interview guide. Yin (2009) suggests the case study researcher should maintain a formal database which other investigators can use to review evidence directly. For this thesis, a database was created and contains all data relating to this study including transcripts, excel sheets used for analysis and diagrammatic representations.

The triangulation of multiple sources of evidence ensures construct validity, and is viewed as a major strength of case study research. Triangulation is used to explore alternative explanations, and confirm validity of the process (Stake, 1995).

Triangulation is particularly relevant to the examination of organisational culture, as different sources of data can target different cultural layers (Yin, 2009). This study uses three types of triangulation to strengthen construct validity by corroborating facts or events highlighted in the study. Methodological triangulation was used in the data collection phase, where data was collected from focus groups and qualitative interviews. Theoretical triangulation is applied during analysis where data is interpreted using research as another unit of analysis. Lastly, supervision provided the opportunity for investigator triangulation, where both supervisors independently reviewed coding and transcripts for units of analysis and findings were discussed.

Positivist researchers criticise the external validity of case study research, stating findings cannot be generalised beyond the case in question. Yin (2009) refutes this, stating case studies are generalisable to theoretical propositions, not populations or 
universes. External validity in case study research is achieved firstly by identifying theoretical relationships (Cresswell, 2014; Stake, 1995; Yin, 2009). Diagrammatic illustrations will be used in this thesis to demonstrate how conceptual claims have evolved. The results chapter will describe how relationships have developed amongst codes, sub themes and theoretical concepts arising from the data and other research.

The discussion section will describe how findings apply to theoretical propositions implicating other situations, outside of this case study, where similar concepts, constructs, or sequences might be relevant. Stake (1995) proposes that findings become valid as naturalistic generalisations develop. He proposes they can resonate experientially with a broad cross-section of readers and this in itself facilitates greater understanding of a phenomenon. Generalisations from this research are likely to be limited to the bounds of the case, namely a tertiary hospital, acute care and/or NZ context. Confidence in findings may lead to new case studies and continue to produce findings related to the same theoretical propositions, which has the potential to further increase external validity of findings.

The constructivist paradigm positions the researcher as a participant in the study (Stake 1995). The researcher is strongly linked to the object of investigation, assuming who we are, and how we understand the world is central in our understanding of ourselves, others and the world (Rubin and Rubin, 2012). Participants' accounts are not so much uncovered, as created by the researcher (Silverman, 2001). This is because qualitative researchers bring their own conscious and unconscious questions, assumptions and beliefs to their work. This is especially important in sensitive research, which can provoke strong emotions and remind researchers of lived experiences (Dickson Swift et al., 2008).

Strategies that strengthen internal validity will address the positivist perspective which assumes truth is absolute and focusses on neutrality, objectivity and facts (Grbich, 2007). Internal validity is strengthened by the process of reflexivity; namely reflecting and critically examining my own thoughts throughout the research process (Holloway and Wheeler, 2010). My own lived experiences, perspectives and preexisting relationships could bias interpretation, especially at the point of analysis. Key authors suggest a research journal is a good strategy to overcome this and can 
be used both for critical reflection and to rigorously document the process (Stake, 1995; Yin 2009). Thoughts, questions and pre-existing relationships were documented throughout the research process in a journal and field notes, and as observations in excel spreadsheets during analysis. This has the advantage of providing an evidence base to respond to challenges of bias following publication. Investigator triangulation and supervision also supported this process.

\subsection{Te Tiriti o Waitangi (Treaty of Waitangi)}

The Treaty of Waitangi is New Zealand's founding document agreed by the indigenous Māori and early European settlers. The Victoria University of Wellington human ethics policy (2016) states researchers:

"Have a responsibility to ensure research conforms to the University's Treaty of Waitangi Statute. As researchers in Aotearoa (NZ), the Treaty principles of partnership, protection and participation should underpin our research relationships in NZ' (p. 5).

This requires consideration is given to the Treaty of Waitangi in healthcare research, which recognising the uniqueness of Māori. A DHB Director of Māori health was consulted at the pilot stage and asked if he felt the research had implications for Māori. We discussed that this thesis is not attempting to understand cultural safety, but what contributes to a culture of safe care. His philosophy is that safe care equates to the standard you would consider to be safe for your own family, in that "what is good for your people is good for my people". This thesis will focus on healthcare professionals' understanding of safe care in their practice and for their 'people', and may involve Māori participants. A patient or whānau member's understanding of patient safety would add further value, but is outside the scope of this research. As NZ provides the context for the case, some descriptions of 'safe care' may include a cultural lens that is unique to New Zealand participants.

Consideration was given to maintaining the principles of the Treaty of Waitangi throughout the research process. The framework provided by the principles of the Treaty of Waitangi was used to identify at the planning stage that additional support, 
input and involvement from the appropriate representative from Victoria University would be sought as required. ${ }^{3}$

\subsection{Conclusion}

Case study is used when the researcher cannot manipulate the behaviour of those involved in the study. Case study is a particularly relevant method for this research as it seeks to understand a broad, complex question within complicated circumstances. Using a case study approach will allow the phenomenon to be examined in detail within the context in which it occurs. The study is strengthened by the examination of patient safety within and across embedded cases to examine the similarities and differences.

A NZ tertiary hospital was selected to provide the setting for this study, and the ED and ICU contribute the embedded units. Doctors and nurses participated in focus groups and organisational managers participated in interviews. Data was analysed using a general inductive approach and propositions generated from the literature review guided analysis. Rigour and ethical considerations were attended to throughout the research process. Findings will be presented in chapter 4 .

\footnotetext{
${ }^{3}$ There were no issues were identified during the research process requiring support and input from a Maori representative at Victoria University. I recognise to fully embrace the principles of the treaty input from a Maori academic would strengthen the research findings in the NZ context.
} 


\section{Findings}

\subsection{Introduction}

This chapter will present the study findings. Stake (1995) suggests sufficient detail of the case is required to provide a "vicarious experience" for the reader (p. 63). Therefore, the context of how patient safety is organised within the case will be provided first. Characteristics of study participants are presented, cognisant of the need to maintain study site anonymity. The main body of the chapter defines and describes themes. Diagrammatic figures, tables and sections of text illustrate and support findings. Participant quotes support findings, and are presented according to the weight of the data. Throughout this chapter, the tertiary hospital acting as the case for this study will be referred to as 'the organisation'.

\subsection{Characteristics of the Case}

\subsubsection{The Organisation}

Field work was undertaken from October 2015 to April 2016, with most focus groups and interviews completed outside of the winter period traditionally associated with increased acute demand.

Quality and safety was the responsibility of an executive director. Furthermore, every directorate had a quality manager responsible for quality, patient safety and risk across a group of clinical areas. Each clinical area (ED/ICU) was allocated a member of staff from the directorate quality team who coordinated the reporting and analysis of adverse events though the organisational governance systems. Quality personnel were located in separate buildings, some distance from clinical areas. Consistent with other NZ DHBs, a number of written procedures detailed the organisational approach to patient safety. 
Safety procedures were designed in accordance with national (e.g. reportable events), organisational (e.g. medicines management) or local (e.g. sedation for a procedure in the ED) schema. The organisation had a detailed and specific reportable event policy. The policy required all staff employed by the organisation to report any instance of actual or preventable patient harm using an electronic database. The directorate operational and medical leader were accountable for the classification, investigation and management of adverse events. Adverse events were discussed at monthly directorate governance meetings which also considered complaints, HDC cases and the risk register. ED and ICU leadership teams composed of a charge nurse manager (CNM) and medical clinical leader (CL) who both reported to an operations manager. The ED and ICU leaders held regular meetings relating to patient safety in accordance with a local governance structure.

\subsubsection{The Emergency Department}

During the period field work was conducted, approximately 130 patients attended the ED daily, and approximately $30 \%$ were admitted to the hospital. The ED was not achieving the $\mathrm{MH}$ shorter stays in ED target, which required $95 \%$ of patients to be admitted, discharged, or transferred from an ED within six hours (MH 2015). The shorter stays in ED target "aims to improve quality and efficiency of care" (MH, 2015 website). Patients could self-present to the ED, or be referred by another service (e.g. ambulance, general practitioner). A registered nurse $(\mathrm{RN})$ with specific training allocated a triage score to patients on arrival. The triage score identified the severity of clinical presentation and the time in which the patient had to be assessed by a doctor or specialist nurse.

Following triage, patients were returned to the waiting room or moved to a secure area within the ED where the majority of clinical activity took place. The area was predominantly open and patient cubicles surround an administration area. The clinical area was split into zones which corresponded with the severity of each patient's illness. The most unwell patients were placed in an area where clinical staff could observe them directly. Correspondingly, the staff-patient ratio was greater in areas of higher acuity. The ED medical specialist in charge could admit patients into an ED assessment unit for observation until a 6-hour limit was reached, but did not require hospital admission under another medical specialist. 
The ED was budgeted to provide medical and nursing staff on each shift. ED specialists were available between the hours of 0800-2400 to supervise and provide direction to the medical team. After this period, a registrar in vocational training and two junior doctors provided medical input with the ED medical specialists 'on call'. RNs worked under the direction of an ACNM (associate charge nurse manager) and senior nurses. A number of senior nursing roles, including clinical nurse specialists and nurse educators, provided education and support to the ED team. The CNM and $\mathrm{CL}$ had recently submitted a business case to increase the number of RNs and security orderlies within the ED. The ED 24-hour roster is available in Appendix 8.

\subsubsection{The Intensive Care Unit}

In the period during which field work was conducted, admissions to the ICU accounted for approximately $20 \%$ of all NZ tertiary hospital activity (ANZICS ${ }^{4}, 2015$ ). Patients were admitted following referral for tertiary level care, complex elective surgery or from in-patient wards. Acutely unwell tertiary level patients were usually retrieved directly to ED or ICU by air. Therefore, geographical context, weather conditions and capability at the patient's point of origin presented unique patient safety challenges. Hospital in-patients were retrieved by a specialist nurse-led rapid response team. On arrival to the ICU a patient would be allocated to one of 18 bed spaces.

Each ICU bed space had a dedicated RN. There was a registrar available in the ICU 24/7, with a consultant on 20-minute call back between the hours of midnight and 0800. Specialist equipment, such as ventilators and vital signs monitors were available at each bed space. A technician provided oversight of additional equipment located in a designated area within the ICU, operated within a 'closed model'; that is, only the consultant on call had the admission rights to the unit. An ACNM and supervisory senior nurse split the geographical area of the ICU, and RNs at each bed space worked with their support. The ICU had senior nursing staff designated to education and quality roles including nurse educators. Furthermore, retrieval services within and external to the organisation were provide by ICU personnel. The 24-hour ICU roster is in Appendix 8.

\footnotetext{
${ }^{4}$ Australian and New Zealand Intensive Care Society
} 


\subsection{Participant characteristics}

Participants were recruited from a variety of roles and settings within the case in accordance with the study design. Six focus groups were conducted at the sharp end of the organisation, four in ED and two in ICU. The number of participants in the six focus groups varied between units (Table 2). Access to participants was unproblematic, and homogenous segmentation was supported by the ED gatekeepers. In the ICU, issues outside of my control prevented focus group homogeneity. Furthermore, access issues prevented a separate focus group for bedside ICU nurses.

Characteristics of the 39 focus group participants are outlined in Table 2. Most of the participants were experienced clinical staff, although three of the six ED nurses had been a registered nurse (RN) for less than two years. Nursing staff typically held posts within the organisation for the longest period. Senior doctors had more varied clinical experience primarily gained in organisations in NZ and the UK, with a minority having worked in the US. Senior doctor groups included both senior medical officers (consultants) and senior doctors in training (registrars). The majority of focus group participants $(n=56)$ were senior doctors $(n=31 / 55 \%)$, followed by senior nurses $(n=18 / 32 \%)$ and nurses ( $n=7 / 12.5 \%)$. Gatekeepers participated in medical and ED senior nursing focus groups. The ICU nursing gatekeeper did not attend the focus group, and a strict time limit was enforced on nurse participation. ED senior doctors comprised the largest group of participants. The gatekeeper requested an additional session, stating clinical staff needed more time to discuss a "very important topic" (EDSMO10). The second ED doctors' focus group also provided the opportunity to increase rigour in the data through member checking. 
Table 2: Characteristics of participants in focus groups

\begin{tabular}{|l|l|l|l|l|l|}
\hline $\begin{array}{l}\text { Focus } \\
\text { Group }\end{array}$ & $\begin{array}{l}\text { Professional } \\
\text { Group }\end{array}$ & $\begin{array}{l}\text { Number of } \\
\text { Participants }\end{array}$ & $\begin{array}{l}\text { Years of } \\
\text { experience } \\
\text { (mean, } \\
\text { median) }\end{array}$ & $\begin{array}{l}\text { Observer } \\
\text { present }\end{array}$ & $\begin{array}{l}\text { Time } \\
\text { (mins) }\end{array}$ \\
\hline $1 \mathrm{a}$ & ED senior doctors & 12 & $15.9,16.5$ & Yes & 29 \\
\hline $1 \mathrm{~b}$ & ED senior doctors & 12 & $15.9,16.5$ & No & 40 \\
\hline 2 & ED senior nurses & 7 & $13.7,14$ & Yes & 70 \\
\hline 3 & $\begin{array}{l}\text { ED registered } \\
\text { nurses }\end{array}$ & 6 & $2.8,175$ & No & 28 \\
\hline 4 & ICU senior doctors & 7 & 15,12 & No & 59 \\
\hline 5 & ICU senior nurses & 5 & $13.7,15$ & No & 26 \\
\cline { 2 - 6 } & ICU RN & 1 & 2 & No & 26 \\
\hline
\end{tabular}

Four members of the executive team were approached to participate in this study. Two agreed to be interviewed and two declined. Of those that declined, field notes state one was unable to participate due to "personal circumstances". The other advised that patient safety was "not part of their role". In addition, a manager with responsibility for one of the clinical areas agreed to participate at a gatekeeper's request. The three managers who participated in this study had previously held clinical healthcare roles, and interviews lasted thirty to forty minutes $(n=3)$.

Where the term participants is used in this thesis it refers to occupational groups working in roles and settings across the case. Where findings relate to both doctors and nurses, they are attributed to clinicians. A unique identifier, denoting the occupational group of a participant and a number (Table 3 ) accompanies quotations. To maintain confidentiality, an appropriate generic term is used where participants or field notes refer to identifiable roles, staff or patients. Participants who took part in interviews and focus groups are listed below in Table 3. 
Table 3: Participants unique identifier

\begin{tabular}{|l|l|}
\hline Staff group & Unique identifier (followed by participant number) \\
\hline ED Senior Doctor & EDSDR \\
\hline ED Senior Nurses & EDSRN \\
\hline ED Registered Nurses & EDRN \\
\hline ICU Senior Doctor & ICUSDR \\
\hline ICU Senior Nurse & ICUSRN \\
\hline ICURN & ICURN \\
\hline Organisational Managers & OM \\
\hline Researcher & JW \\
\hline
\end{tabular}

\subsection{Defining Harm}

Participants described patient safety as the reduction of clinical risk that could lead to harm. Furthermore, participants proposed patient harm was inevitable within the organisation and inaction in the face of safety problems was common. Participants suggested harm would continue to occur within the prevailing culture, with harm described as either acceptable or unacceptable. Participants described acceptable harm as the harm occurring in spite of all necessary actions being taken by all members of the organisation, with the patient's informed consent - for example, a drug reaction to life saving treatment(s). Participants described unacceptable harm as:

- harm attributed to healthcare process (iatrogenic harm)

- harm attributed to deviant acts

- harm attributed to latent conditions within the case in question and/or wider healthcare system. 
The following quotes were typical across all participant groups and illustrate the belief that harm is inevitable:

\begin{tabular}{|l|l|}
\hline Managers & $\begin{array}{l}\text { "We do a whole lot of things to patients, and therefore the potential } \\
\text { to do harm is high, even if it's unintended" (OM2) } \\
\text { "Through their journey, we take every effort to ensure that [patients] } \\
\text { don't come to harm, and that can be anything from medical } \\
\text { misadventure, to we didn't get it quite right" (OM3) }\end{array}$ \\
\hline Doctors & $\begin{array}{l}\text { "[Patient safety] is on a continuum. There are risks both from [the } \\
\text { patient's] presentation and what we need to do to help them, our } \\
\text { role with our safety hat on is to reduce the risks on both sides of the } \\
\text { equation as much as possible." (ICUSDR1) } \\
\text { "All we can do is reduce it to the lowest risk anyway. You can never } \\
\text { make things 100\% safe" (EDSDR2) }\end{array}$ \\
\hline Senior Nurses & $\begin{array}{l}\text { "Risk and safety are sort of the same" (EDSRN3) } \\
\text { "The truth is it isn't safe most of the time" (EDSRN5) }\end{array}$ \\
\hline
\end{tabular}

\subsection{Safety Capability}

Participants indicated patient safety was influenced by safety capability. Safety capability was the theoretical concept developed from the analytical process, and is defined as the power or ability to provide safe patient care. Participants indicated that two themes contributed to safety capability within the organisation: anticipation and vigilance; and a resilient culture. Participants had developed strategies to anticipate and remain vigilant to risk, identified in the sub themes of surveillance, being proactive and timeliness. Furthermore, participants identified a resilient culture, underpinned by the sub themes of building blocks, collaboration and trust, and balance, was required to mitigate the risk of harm (Figure 4). The full coding frame is available in Appendix 2. 
Figure 4: Theoretical model developed from the coding frame

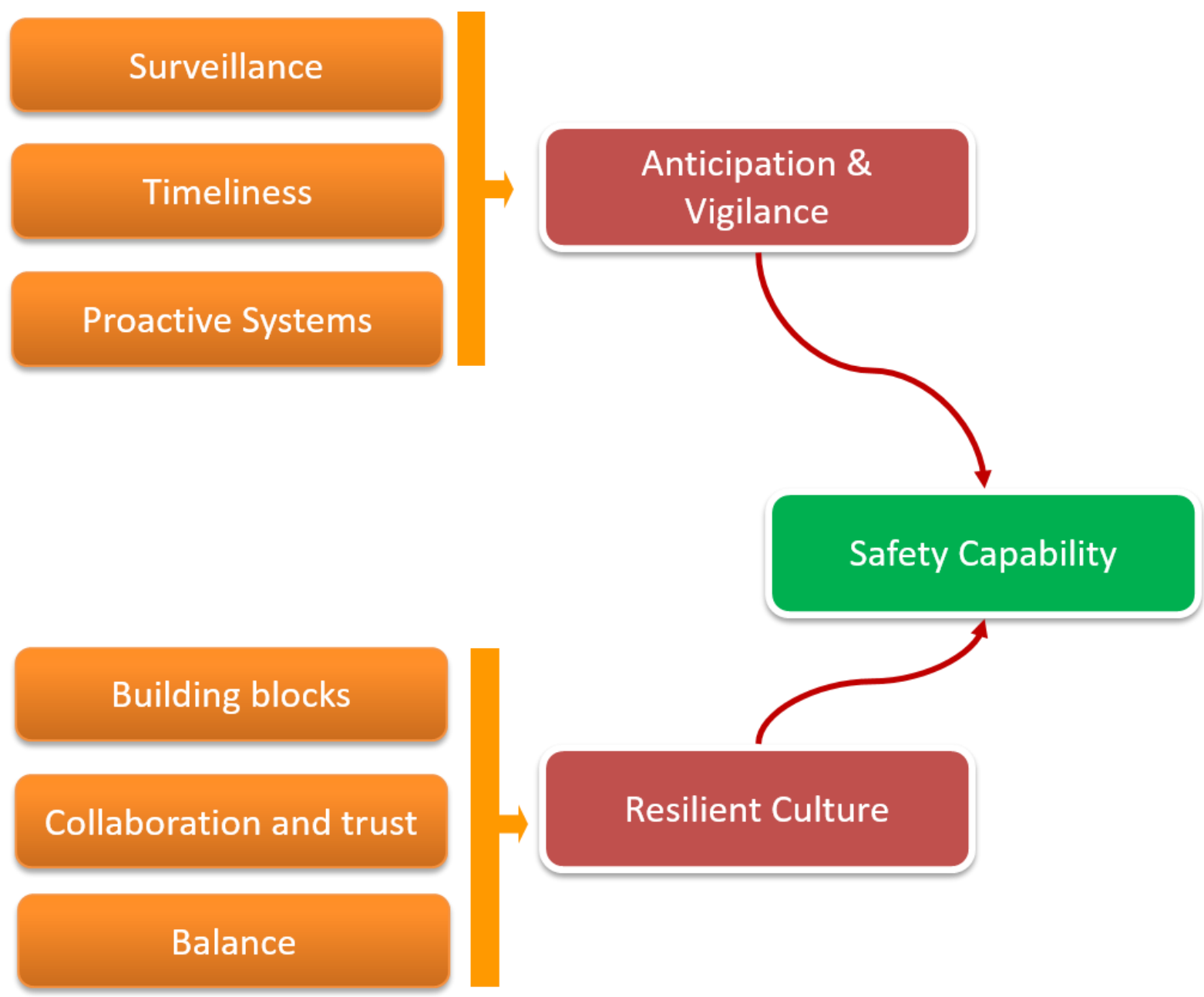




\subsection{Anticipation and Vigilance}

Anticipation and vigilance were interdependent constructs within the organisation. A number of systems and processes had been developed to anticipate and remain vigilant to events that could lead to patient harm. Systems anticipated, recognised and responded to risk, and some were more successful than others. Systems included information gathering, organisational control structures and written procedures. Three sub themes contributed to the theme of anticipation and vigilance: surveillance, timeliness, and a proactive approach.

\subsubsection{Surveillance}

\section{"We can anticipate there are going to be problems." (OM3)}

Participants identified specific safety tools that identified and graded risk within the organisation. Surveillance tools were either of a proactive nature to assess and respond to risk, or reactive in reviewing retrospective clinical data and making suggestions to prevent reoccurrence. Managers placed high value on surveillance tools they used for "risk mitigation" (OM2), and tools that monitored organisational capacity or resources in real time were particularly valuable to them (e.g. bed occupancy, performance against the shorter stays target). Trend Care $\AA$, a tool that predicted required nursing hours in each clinical area every shift, was cited frequently. Managers indicated the redeployment of nurses was a common strategy to optimise patient safety conditions within the organisation and senior nurses and managers used TrendCare ${ }^{\circledR}$ to inform redeployment decisions. Participants spoke about a number of meetings across the organisation where staff from roles and settings gathered to share surveillance information. Daily bed meetings were highly valued by managers and senior nurses alike for this purpose. Both groups suggested bed meetings contributed to risk mitigation across the organisation:

"We have our daily bed meetings sometimes twice a day (...) where we can anticipate there are going to be problems." OM3

Participants identified several meetings where the key function was analysis of retrospective data deemed relevant for safe care. Membership was dependent on occupational group (e.g. doctor's mortality and morbidity meetings), or job 
description (e.g. directorate governance meetings) and the forum chair could also invite members to participate. Meetings were informal or had formal terms of reference. In general, clinicians valued forums where extreme events deemed to have potential for "learning" (ICUSDR2) were established and discussed. Clinicians indicated they valued learning from effective action and behaviour in extreme situations, as well as adverse events, such as through weekly ED simulation training. Clinicians indicated they believed forums led by management directives were often driven by a desire to improve efficiency rather than safety. For example, ED clinicians suggested a CEO forum for patient safety, similar to an efficiency savings forum they had been invited to, would be beneficial. Clinical staff expressed they believed 'the organisation' would not be willing to engage in a debate about patient safety, largely because a lack of funding existed within the healthcare system:

"That's not through our bosses trying, I think it's coming from, but I think it's broader than the DHB I think they just don't have it in their budget." (EDRN4)

Clinicians suggested that managers were often unable to action what was required due to limited budgets. Commentary from one manager supported their view:

"There are minimum standards, and there are gold plate standards, and we can't afford gold plate." (OM2)

Managers suggested the reportable event system was the most important surveillance tool for patient safety, described by one manager as "the bottom line" (OM3). Managers also valued surveillance data regarding the "top 10 clinical indicators" (OM1), such as HQSC prescribed quality safety markers (falls, medication errors), and mortality data. Descriptions of the "patient safety system" (OM2) included a framework that evaluates "potential risks, inherent risks.... and risks that were underestimated" (OM2). Furthermore, surveillance tools were described as belonging to a "clinical governance framework" (OM2 and OM1) in which patient safety was situated and managed.

ED clinicians also discussed a governance framework; the national ED framework of quality measures (MH, 2014), describing this as an important surveillance tool. However, clinicians indicated organisational conditions, such as sub optimal IT and reporting systems and a lack of human resources for audit, prevented the framework from being successfully implemented. The dominant view across participant groups 
was that surveillance tools are not easy to use. Clinicians indicated this had led to disengagement with some safety systems, as can be seen in this comment:

'We've got (..) 139 God damn things [to measure] going up every single day." (EDSDR5)

Managers viewed advocacy as an important facet of their role, suggesting surveillance data allowed them to "represent" (OM1) patients on safety issues, especially when additional funding was required to resolve or mitigate risks. One manager stated that the organisation's risk register was an important surveillance tool for this purpose:

"If you've still got a level of risk that's not tolerable (....) we do have a responsibility to raise that up to the board (....) you can't continue to operate a system that's under pressure and is potentially creating patient safety issues." (OM2)

All of the managers in this study placed importance on near miss reports, and this comment was typical:

"We report that we support and reinforce a low threshold for reporting and that we are as interested in the near misses, (....) as much as we are in those serious events where it's really obvious that there's been an adverse outcome." (OM3)

Conversely, clinical staff believed important data about risk was often ignored, suggesting "they don't even look at them" (SEDRN2). They indicated management action occurred in response to rare events, rather than frequent individual ones. This was particularly evident in ED focus groups:

“That psych watch we've missed, something happens then it's an adverse event, but actually it was a [near miss] for about the last 20." (SEDRN3)

"We all know the concerning issues are those [near misses] that keep happening.... every single day.... It's those things that just keep accumulating, and aren't getting addressed." (EDSRN1)

Clinicians suggested resources considered essential for safe care were "repeatedly requested" (EDSRN1). Furthermore, they proposed an adverse event, HDC case or 
negative press story was required to generate management action. This comment from an ICU consultant summarised the dominant clinician perspective:

"It's easier to get resource to fix a rare problem that has caused a single bad event than introduce a concept of improvement of care that makes sense to everyone and has potential for great health gains." (ICUSDR2)

Although rare organisational incidents were addressed, clinicians indicated that the potential for the adverse event to reoccur remained.

“We don't actually learn from our mistakes, and that really worries me because we make the same mistakes all the time (....) We've been in the news; we're creating some of those HDC cases ourselves unfortunately. Even if you look at it now (......) three years later hand on the heart I can't say this is not going to happen again." (EDSDR10)

Clinicians identified two surveillance systems they considered to be proactive: triage and the early warning system (EWS). Clinicians indicated both systems identify patients at risk of deterioration. Measurement of vital signs, described as "monitoring" by many clinicians, was strongly linked to patient safety in both of the acute care environments. However, clinicians placed equal importance on skilled intervention, when vital signs were outside acceptable limits

Participants provided many examples where EWS had improved safety capability within the case in question including: for example, reduction of adverse events on the general wards following ED discharge, and timely mobilisation of critical care expertise to the patient's bedside. Senior clinicians suggested the EWS had provided a common language for patient acuity, positively contributing to safety culture within the organisation. The following comment from a senior ICU nurse, was typical:

"We're so much more acutely aware of the deteriorating patient with our [EWS] system and patient safety out on the wards... Either we need to bring patients here, or we need to find strategies to help them out on the wards, it [patient safety] actually goes beyond these walls." (ICUSRN1)

ED clinicians identified triage as their primary surveillance tool. Clinicians in this study used the strategy of "re-triage" (EDSRN4) to assess and stratify risk at times of 
operational flux. Participants indicated re-triage reduced the risk of harm from crowding, when they were unable to meet original triage times. The importance of retriage for patient safety was evident in this comment from a senior ED nurse:

"A patient is always at risk of something happening. For example, someone has an ectopic pregnancy and it was missed...that patient was at risk a. because they were in the corridor, and b. because they probably weren't seen within 30 minutes when they were triaged. Risks always add up and the end." (EDSRN5)

\subsubsection{Timeliness}

\section{"There's a difference between efficient and effective care to speed. Speedy care is not what we are about." (SEDRN1)}

Participants described timeliness as timely recognition and intervention, and having time to provide care within the right timeframe (e.g. triage, sepsis protocols, FastTrack pathways). Participants suggested harm occurred when they failed to put "patients on the right journey" (OM3) in a timely manner and clinicians described rushed care as unsafe. Rushed care, described as speedy, was characterised by a lack of time for collaboration and surveillance, the inability to apply minimum standards, and being forced to work around organisational rules and procedures. Clinicians indicated that 'speedy care' occurred daily within the organisation, and descriptions typically included feeling overwhelmed:

"Safety is being able to provide care where you're not too stretched, and trying to be in too many different places at once, and feeling over worked or over pressured." (EDRN4)

"[Safe care is] to provide timely and efficient care, not to the best of our ability but to the best for the patient, because our ability may be hampered sometimes by system and processes around us." (EDSDR11)

Several examples of unsafe care relating to timeliness were provided by clinicians. They frequently mentioned delays in elective cancer treatment and cardiothoracic surgery, delays in aeromedical retrieval for time sensitive intervention (e.g. head injuries) and delays in MET call activation as antecedents to harm. Barriers to timeliness included poor access to information (e.g. diagnostics) and an inability to 
access decision makers. ED participants and two managers indicated they valued the $\mathrm{MH}$ shorter stays target. ED clinicians supported the target to act as a forcing function for "timely assessment and intervention" (EDSDR8) by speciality services. ED senior nursing staff suggested the target made their service visible, acting as an indicator for problems within the TH or primary care that required investigation or investment. Conversely, ED clinicians proposed the ED target could also reduce safety capability, indicating harm is more likely when speedy care increased to achieve the target. Examples included working around organisational processes, and discharging patients from ICU at night to accommodate a patient.

Doctors suggested they "prioritise" which interventions and improvements they had the "time to buy in to" and described feeling overwhelmed (ICUSDR1).

Organisational safety interventions were not adopted if they were viewed as cumbersome and/or impacted on timeliness. Safety improvements were considered to be successful by clinicians if the change and implementation processes were perceived to be efficient and effective. Clinicians suggested current approaches to quality improvement meant "having 20 things on the go, none of which actually get any progress, or any buy in or only sort of half done in a half arsed way" (ICUSDR1). They suggested this approach had unintended consequences for patient safety, as in this comment:

"Changes made to a system in terms of safety often lead to a drop in safety outcomes for a period of up to 2 years because of the change in practice introducing or slowing the system down, introducing new error, people learning that new error, modulate." (ICUSDR2)

Change fatigue was attributed to constant organisational restructure, a lack of resources, evolving models of care, and/or multiple technological systems that did not interact. The perception that the organisation was prioritising short term fixes to save money over changes to address patient safety concerns over the long term was evident in all focus groups.

Clinicians discussed that care provided in a rushed manner significantly reduced the time they had to communicate with patients and their family/whānau. Clinicians indicated communication keeps patients safe from a "psychological point of view" (ICURN1). Clinicians suggested this time is crucial in acute care environments so 
that one can understand the tolerable risk of individuals (ICUSDR2). This time was highly valued in ICU where patients required significant intervention and had a high mortality risk. Clinicians proposed safe care is underpinned by informed consent, where a patient decides which risks are tolerable for them as individuals. The importance of time for communication with patients and families was summarised by ICUSDR2:

"[talking to patients and families] takes into account ...the difference between the general and the particular, the personal approach to risk, the personal approach to value of an outcome or a particular intervention, and you can do that by understanding the person."

\subsubsection{Being Proactive}

\section{"It's not about winging it" (ICUSRN2)}

Participants suggested the proactive management of clinical risk was essential for safe care. Managers suggested written procedures that direct care (e.g. protocols, procedures) are the tools that achieve this within the organisation. Clinicians appreciated procedures designed for timely intervention (e.g. FastTrack pathways for myocardial infarction or sepsis), but indicated they do not exclusively rely on written procedures:

"There's protocols and fast tracks and things like that, it's great you just go to a piece of paper and it tells you what to do and there's always someone around that you can ask, what should I do?" (EDRN1)

ED Doctors proposed "risk stratification" (EDSDR5) is an essential process of skilled assessment and clinical judgement that occurs before the right clinical pathway can be activated. Furthermore, new information, such as diagnostics and/or changes in clinical condition alter which written procedures are required at any point in time. Clinicians stated the number of simultaneous interventions in acute care environments require adherence to multiple policies at any given point in time. Furthermore, they proposed these conditions can add to, rather than minimise the potential for human error. Clinicians indicated working outside of "what is predefined" (EDSDR10) was common. Whilst acknowledging working outside 
predefined rules can contribute to patient harm, clinicians suggested harm also occurs from blindly following protocol-based care. A proactive approach was considered to be particularly important when patients have an atypical presentation, as there is an increased risk of "misdiagnosis" (EDSDR5):

"The inherent risk with pathways and guidelines is that the first step is recognition. If you don't recognise something you're never going to start the pathway. Now does that mean it's unsafe if you don't recognise it, well that's open to debate because very few things present like we've been taught they present in a text book." (EDSDR10)

Notably, managers had a different view, and considered breaking written procedures a deviant act:

"You can have the frameworks there but if people aren't working to that and making it part of what they do every day then it's a waste of time." (OM2)

Clinicians in this study suggested organisational conditions are increasingly factored into risk stratification. Clinicians described a complex process where risks posed by an individual patient's pathophysiology were considered within the organisational context at a given point in time. Participants across groups described a continuous state of "flux" (EDSRN5) was a dominant feature of the organisation. Participants adapted by "fighting fires" (ICUSDR5), described as a skill gained from exposure and involvement in extreme situations.

Senior nurses and managers viewed fire fighting as a badge of honour, emphasising harm is prevented by proactively working around, and within suboptimal conditions. Fire fighting skills were seen to reduce clinical risk in the context of a "background of chaos" (EDSRN2), by "around situations" (ICUSRN5), with one participant concluding "the truth is; it isn't safe most of the time" (EDSRN7). Fire fighting patched risks in daily routines, so participants could keep working. Fire fighting was also valued by senior ICU nurses, and ICU doctors with MET team or ED experience, who suggested "contingency mode" (SRNICU2) existed in a number of clinical areas within the organisation. The following comment was typical:

"It's not about winging it, it's about relying on your intuition and a bit of gut feeling about how far we can stretch, and what wouldn't be ideal if you were planning 
forward, but at that point in time is possibly your only solution. You can still make it safe by putting other things in place." (ICUSRN2)

\subsection{Resilient Culture}

The second theme developed from the data was resilient culture. Resilience is the organisation's ability to maintain and/or regain a dynamically stable state. In order to achieve this, participants relied on human process and relationships, especially in the presence of continuous demands. Three subthemes contributed to resilient culture: building blocks, collaboration and trust, and balance. The following section presents findings relating to these subthemes in detail.

\subsubsection{Building blocks}

\section{"Patients want to have confidence that you have staff, and they're well trained and equipped to do the job." (OM2)}

Participants suggested three building blocks were essential for a resilient culture: the right staff with the right skills; the right leaders; and the right environment. The following comment was typical:

"Having the building blocks in place is really important no matter what culture you've got." (ICUSMO1)

\subsubsection{The right staff with the right skills}

The need to match human resources against demand was described as a particular challenge within the organisation, especially at times of flux. Clinicians suggested a number of factors contributed to inadequate staffing levels within the organisation including increasing patient complexity, staff burnout, competing health targets and inadequate budgets. They further suggested core resources had not increased alongside acute demand. Common examples of patient safety events relating to inadequate resources were cancelled operations and poor skilled staff ratios associated with adverse events. Clinicians proposed patient safety was compromised by a lack of resource "buffer" (ICUSDR2) in the organisation. They suggested clinical staff were required to move around the organisation at times of flux, with little opportunity for recovery. The following comments were typical: 
"I think we do have a culture of safety. We organise within the department... and we try to practise in a safe way... it's just sometimes it's money, isn't it? We need more staff." (EDSRN5)

“Everyone's right at capacity so just one or two sicknesses or one or two problems and the [elective] cases start falling off. They're all different parts of the chain but because everything is working at 95\% you've no buffer for problems, and because the team's inter-related, one problem affects the whole system." (ICUSDR1)

The impact of the staffing perceived to be inadequate was described as multifaceted, as in this comment:

"If you are running the shift 2 staff short, the impact is going to be anything from the ability of [the junior doctor] to manage an increased case load to, the time [nurses] are able to give to the patient in terms of general care, frequency of vital signs, frequency of documentation and then obviously, the speed at which particular treatment can actually be delivered." (EDSRN3)

There was a perception amongst clinicians that managers did not understand the resources required to keep patients safe. For example, ICU doctors suggested a common misconception was that ICU had a "luxurious situation, mostly because we have got, to everyone else's standards, ridiculous resource of nurses" (ICUSDR4), stating they worked hard to "protect" (ICUSRN1) nurse patient ratios. Clinicians suggested staff numbers were reviewed without an appreciation for the importance of skill mix, proposing this was as important as the number of staff. Clinicians in ED and ICU stressed the skill mix of the team was the most important consideration in acute care areas. Managers also appeared to value a team approach and measured skill mix of clinical staff using formal methods including "credentialing 5 " doctors and "PDRP leve/6" (OM1) of nurses. As one manager suggested:

\footnotetext{
${ }^{5}$ Credentialing is "a process used in NZ to assign specific clinical responsibilities (scope of practice) to health professionals on the basis of their training, qualifications, experience and current practice, within an organisational context. This context includes the facilities and support services available and the service the organisation is funded to provide. Credentialing is part of a wider organisational quality and risk management system designed primarily to protect the patient." (MH $2001 \mathrm{p} \mathrm{2)}$

${ }^{6}$ PDRP is the professional development and recognition programme for nursing staff in New Zealand.
} 
"[patient safety requires] adequate numbers of nursing staff, do we have the right skill mix, do we have also the doctors that are on the shift, who's managing the shift, the ACNM (associate charge nurse), (the person with responsibility for patient flow), which SMO is in charge, which registrar, which house officers are working this shift?" (OM3)

Clinicians suggested adequate skill mix prevents the team from making errors, by alerting individuals to actual or potential risk. During periods of high demand, or diluted skill mix, this was viewed as of particular importance. Clinicians suggested in an unfamiliar situation juniors may be unaware of the degree of risk. Clinicians suggested patient safety culture required the team to identify, discuss and manage risk, proposing the team is accountable for decisions that result in sub-optimal outcomes.

Participants indicated the right skill mix for patient safety requires continuous education and training. Although common service specific courses were suggested, clinical staff emphasised the importance of non-technical skills training. Training with an emphasis on teamwork, notably simulation and crew resource management (CRM), was highly valued. Participants emphasised the importance of specialist, skilled support to facilitate this process, provided by "go to people" (EDRN4). The role of 'go to' people was supportive; they generated learning, provided specialist clinical oversight and were skilled firefighters. Managers termed the process "peer supervision" (OM3) and stated effective teamwork is monitored within the organisation's governance framework.

\subsubsection{The right leadership}

The second building block is concerned with the right leadership. ED clinicians proposed "anyone can be a leader" (EDSRN1). As one senior nurse stated:

"What you do is you instil the leadership in all levels of the system ...so it's not one person leading everything, you've got leadership all the way through every level... so that's a safety mechanism all the way down." (EDSRN5)

A requirement to integrate patient safety into "everything we do" (EDSRN3) was suggested by participants across roles and settings within the organisation. Participants characterised safety leadership as visible, trustworthy and responsive. 
Leaders were described as good communicators who made staff feel valued, supported, empowered and who cared for and understood conditions at the coal face'7. This perspective was particularly evident in a discussion by junior nurses about the organisation's leaders:

"In the middle of winter, I would love to just put one of them on a bed in the corridor for 8 hours (...) I think a lot of them don't quite understand how hard it is, and if they just sat there and actually watched they might change things a bit." (EDRN4)

Participants suggested a key facet of leadership for patient safety was to ensure staff safety, in particular by preventing emotional "burnout" (OM1). High value was placed on leaders who provided psychological and professional support to prevent "people going home exhausted" (ICUSRN1), as it was "getting harder to look after people's mental health" (ICUSRN5). Clinical staff in both clinical areas suggested burnout was an increasing concern. Managers said they would respond to requests for help and indicated they were unaware that burnout was a significant issue:

"(The organisation) has to have an environment where staff can put up their hands and say enough." (OM2)

The role of organisational managers was perceived by clinicians to be supportive, rather than to control or provide direct oversight. Clinicians valued managers that trusted the team, led by the CL and CNM of each clinical area to deliver safe care. This was particularly evident in a discussion about the role of the ED ACNM:

"It's a wee bit like an air traffic controller, there is massive risk for an air traffic controller, there is planes everywhere with hundreds of people on and that person kind of pulls it all together, but in that they're trusting that each of those pilots know what they are doing." (EDSRN2)

Discussions regarding leadership were extended by clinicians to the wider healthcare system and external agencies. Clinicians suggested the national reportable event policy had a negative impact on safety culture, which was particularly evident in this comment:

\footnotetext{
${ }^{7}$ The 'coal face' is a colloquialism that means doing the work involved in a job, in real working conditions, rather than planning or talking about it. (Cambridge online dictionary 2016)
} 
“The safety culture generates so many reportable events you've got people running around in circles trying to classify them, justify them, do analysis of them to find the one individual who the finger can be pointed at to make them stop doing it again. No one's being proactive, everyone's being reactive." (ICUSDR3)

In order to develop strategies and policies that prevent harm, clinicians recommended that the context of individual organisations, and the wider healthcare system, must be accounted for. The HQSC annual adverse events report was seen as reactive, negative and unique to healthcare by clinical staff, with one RN suggesting:

"they certainly don't put a list of what (the current prime minister has) done wrong during the year" (RN3).

Clinicians acknowledged patients had the right to information, but suggested having the death of a loved one debated in a newspaper caused further harm to families/whānau. They suggested a focus on negative events eroded trust between clinicians and patients, and indicated information in the public arena, such as newspaper stories and HDC decisions, did not account for contextual conditions. Public discussion which focussed on how doctors and nurses had contributed to patient harm was perceived to be personally and professionally distressing. This was particularly evident when clinicians discussed HDC breaches and the newspaper articles that followed:

"When that happens again next time it's the clinicians that are under fire. Whether it's the triage nurse that works substandard environment, or the junior doctor because he's not supervised by SMO who's busy with 20 other sick patients." (EDSMO5)

"They never say, they saw this many people, they diagnosed this many (...) they got this many people to ICU and this many people survived to discharge. They never publish the good stories." (EDRN4)

Ownership of patient safety within the organisation was unclear. Patient safety was acknowledged as everyone's business, and most clinicians indicated ownership of risk was an essential component of their role: 
"I will take on my patient's risk as my own risk...everything I do, and change, and make decisions about is a risk for my patient and for me and for my team." (ICUSDR5)

ED clinicians proposed risk stratification was a key facet of everyday work. However, clinicians more broadly suggested they did not have the time or skills required to resolve patient safety concerns:

"We come up with a problem and then we're meant to solve that problem as well which is actually outside our field. I should be able to just practise safely knowing that things are safe.... We trained to be doctors." (EDSDR5)

The organisation's figure head was identified by clinicians as the person who should lead and be accountable for patient safety. They repeatedly made statements regarding a "lack of ownership" and "lip service" (EDSMO10) regarding patient safety. Patient safety was described as belonging to a number of non-specific groups including "everyone", "quality", "service managers", and "clinical leads". Managers indicated other occupational groups did not always take ownership for patient safety and proposed they were "engaging the workforce" (OM3) into a "culture of improvement" (OM1). ED doctors suggested a "patient safety officer" (EDSDR6) should be employed by each department to resolve systems issues, using a "proper process" (EDSDR2) akin to the traditional scientific method. The following comments were typical:

"It's everyone's role but it needs to be led and communicated that it's everyone's role and clearly steered and I don't see that steering." (ICUSDR1)

"In communication patients need to be put first not targets, not negative outcomes, not bad publicity, the patient needs to be seen through all of that. And unless you do that, you are going to have some difficulty with culture." (EDSDR10)

Poor leadership was consistently related to "bad behaviour" (OM3), characterised by a lack of collaborative, collegial or respectful characteristics or prioritising money or reputation over patient safety. Good leadership was described as synonymous with emotional intelligence, collaboration and motivational skills. This comment was typical: 
"People get promoted based on their technical skill as opposed to their leadership ability, the emotional quotient isn't there so they get things to happen, but the relationships that endear people to want to do their best doesn't exist." (OM3)

Poor behaviour was described as a particular challenge in medicine, and participants related it to "historical practice" (OM3), and subspecialist culture. ICU doctors explored the notion that medical training encouraged a culture of independence, rather than collaboration. They suggested the experimental learning culture of medicine compromised patient safety. The impact of historical practice and bad behaviour was particularly evident in this comment:

"As a junior, there are departments in the hospital where you would avoid talking to your consultant for fear of bothering them or getting reprimanded that you're kind of wasting their time.... I've seen simple procedures and things get done by people who had never seen or done it before and they looked it up and they YouTubed 8 it.... because they felt incapable of talking to someone more senior." (ICUSDR2)

\subsubsection{The right environment}

The final building block was the "right environment" (OM2, EDSDR5). Managers used this term to describe a positive organisational culture. However, clinicians used the term to describe the physical environment. These comments were typical:

"Patient safety is about the environment. Making sure the equipment is there for the skills and knowledge that are in the heads and hands of the clinicians." (EDSRN3)

Clinicians' descriptions of a safe physical environment included basic working infrastructure, including toilets, taps, waiting room chairs, and basic equipment such as oxygen saturation probes. Participants suggested other areas within the organisation also had an unsafe environment, as demonstrated in this comment:

"In the (ward) isolation rooms where they've got sick neutropenic patients, they haven't even got a blood pressure machine in each room.... Now this is just ridiculous." (OM1)

\footnotetext{
${ }^{8}$ YouTube is a video sharing service that allows users to watch videos posted by other users and upload videos of their own
} 
ED participants placed high value on having enough space to allocate patients to an area that matched the patients' clinical needs. Senior nurses identified a key factor in patient placement decisions is severity of illness, stating patient movement had become a "large part of our role" (ICUSRN1). They proposed moving patients around their department and the wider organisation redistributed risk. Senior nurses indicated patients were at risk outside dedicated treatment areas:

"Patients are unsafe in the corridor, they can be bumped into by things, they can get lost, they're environmentally in danger." (EDSRN5)

Clinicians identified they used a number of strategies to control the environment. For example, CRM principles were used in resuscitation areas to mitigate the risk of error. Clinicians indicated altering the physical environment was an important strategy at MET calls where specialist equipment and "go to people" were mobilised to deteriorating ward patients. The ED's physical environment was characterised by a lack of space and nursing patients in corridors. Conversely, ICU participants were positive about their physical environment. The difference the physical environment made to patient safety was particularly evident in a conversation with a doctor who had recently rotated from ED to ICU:

"It feels very different, when you remove a patient from emergency or from the ward. I feel much safer here in this (ICU) environment (....) I feel it's safer for my patient and for me." (ICUSDR5)

Clinical staff suggested physical, psychological and behavioural attributes of patients and families/whānau altered the physical environment. They proposed the presence of one person increased the risk of harm when it affected another. Clinicians indicated patient safety was frequently compromised by violent, aggressive or intoxicated ED patients. This was particularly evident in a discussion about late shifts:

"If you have an intoxicated aggressive person in the department that might consume several staff members time...you want your time to be spent with the elderly patient with the fractured hip who's got pain" (EDRN2) 


\subsubsection{Collaboration and trust}

\section{“We can't just work in isolation” (ICUSRN1)}

Collaboration and trust were described as highly regarded interdependent constructs. Collaboration was viewed as an antecedent to teamwork and a value underpinned by trust:

"I think [collaboration] enhances patient safety. It's a team game medicine. Time and again intensive care specialists see the (bad) results of silo mentality on patients". (ICUSDR3)

Descriptions of teamwork were functional, where the multidisciplinary team met and individuals performed the role specified in their job description. Collaboration was essential for good teamwork to occur, and included responsiveness, respectful communication and every contribution was valued. Participants in roles and settings across the organisation discussed collaboration with patients. As previously stated, clinicians viewed patient collaboration as an important strategy to gain informed consent ( $p$ 85). Managers associated collaboration with consumer confidence (OM2) in the safety of the healthcare system. Managers used terms traditionally associated with the quality improvement movement to describe patient collaboration such as "co-design" (OM1) and "patient experience" (OM3).

Collaboration was described positively within speciality teams (ED/ICU). Collaboration with other speciality groups was usually described negatively. For example, ICU doctors attributed culture lacking collaboration to the "single organ doctor" (ICUSDR2, ICUSDR3). Participants indicated poor collaboration was common, especially when there were competing beliefs. One manager summarised the views of the majority of ED participants:

"What's happening in ED is not strictly an ED goal or problem; it's the whole of hospital problem. Trying to get the medical specialities to understand that it's not an ED problem, but it's their problem, that's a whole culture change." (OM3)

Descriptions of poor collaboration clinicians provided were characterised by "doctor's arrogance and bad behaviour" (EDSDR2). For example, the decision to decline ED 
referrals was frequently discussed and described as "very personality driven." (EDSDR9). Ultimately clinicians suggested poor collaboration was:

"A cultural problem that we (the organisation) have." (EDSDR6)

Managers, doctors and nurses suggested if multidisciplinary groups gathered "around the table" (ICUSDR2) they could plan for safer care. Participants indicated safe care planning required an exchange of divergent perspectives:

"The Health Minister alludes (to the fact) that clinicians are the ones that are going to make or drive change." (OM1)

"We need managers to tell doctors when to stop spending money. If doctors aren't going to be responsible financially...health care spending could be infinite" (ICUSDR3)

Collaboration between managers and clinicians was described as poor. Clinicians acknowledged "tension exists" (ICUSMO2) between the two groups. Senior clinical staff described "a constant battle" (EDSRN1), where patient safety concerns had to be "justified" (EDSRN1). A lack of trust was particularly evident in conversations about TrendCare $\AA$. TrendCare $\AA$ required bedside nurses on the wards to predict nursing requirements for every patient each shift. A senior nurse suggested TrendCare $\AA^{\circledR}$ data showed that nursing resources varied across the organisation:

"[The tool] is showing some valid and reliable data. Some wards are over staffed and other wards are grossly under staffed. The organisation [has a] responsibility around human resource, to actually acknowledge that and act on whatever it finds.... otherwise it's pointless." (EDSRN1)

In a later interview a manager indicated they did not trust the data collection process:

"Once we have confidence in the accuracy of the data that we are getting we will feel much more confident when we then we need to pull the staff in. [lt's about] making sure we're doing the rating correctly and that the data is accurate to then be able to use it in a way that then doesn't compromise staff or patient care." (OM2) 
In further comments the manager suggested variability in nurse staffing levels was due to poor management of nursing rosters:

"We have a planned roster to meet what you would anticipate, which you can flex up and down around. But if you don't then actually roster to the plan, or give too many people leave or you've got a whole lot of people off on sick leave, it doesn't mean that the plans wrong, it's how we execute the plan". (OM2)

Informal communication was described as essential for collaboration. Participants across occupational groups used informal communication to prevent harm in day-today operations, especially clinicians. Informal communication included conversations about clinical and individual risks that continuously occurred and were not formally captured in writing. The information gained from informal communication was used to evaluate and alter risk mitigation strategies. Examples included phone conversations, huddles, daily bed meetings and handovers. Informal communication converged from multiple sources and was highly valued at times where clinical staff had to "work around" (EDSRN5) normal processes. Participants used informal communication to manage problems that were not accounted for in written procedures. Clinicians suggested everyone had to trust that the information provided was accurate, and used it to inform decisions about risk:

"Trust to me is a very important bit of good team work, there has to be very clear communication around that as well, so if we go outside what is pre-defined that we communicate that." (EDSDR1)

"If we know (a patient is) not going to be safe wherever they're heading, because of resourcing or staffing or location or visibility or whatever the reason might be, then that has to factor into all the decisions we're making .... We can't just work in isolation." (ICUSRN1) 


\subsubsection{Balance}

"If the ducks don't line up, then something will fall out of the sky." (EDSRN4)

Participants indicated that patient safety existed on a continuum. Care provision was influenced by the ability to balance organisational resources against demand at any given time. Participants proposed that responding to immediate risks in one part of the organisation created unintended consequences elsewhere. The following comments were typical:

"There's so many layers in a big hospital system that it doesn't take much for the system to fall down because of one chink in the armour." (ICUSDR1)

"if one part isn't working the risk all goes to one area." (EDSRN5)

Participants were of the opinion that patient safety required a change in healthcare system planning, funding and strategy. Clinicians proposed long-term planning was essential with acute demand forecast to grow exponentially over the next decade. Participants across roles and settings suggested annual DHB funding models limited strategic planning and were suboptimal for safe care provision:

"We all know that dollars and resources are constrained and the demand is growing and much faster than the funding that we get, so it's how we do the best with what we have." OM2

Clinicians proposed healthcare system imbalance would increase the prevalence of harm without significant action. Managers suggested the "dollars (would) look after themselves" (OM1), if patient safety was optimised, but were resigned to the "stage of development we're at" (OM2). Some clinicians suggested the $\mathrm{MH}$ would not allow imbalance to continue if they were aware of the conditions patients and healthcare professionals experienced. Other participants suggested the $\mathrm{MH}$ was aware of the unsafe conditions created by system imbalance, but strategies at the time were ineffective and ignored concerns.

Participants indicated $\mathrm{MH}$ healthcare targets created imbalance and did not guarantee patients would be safe: 
"if we meet targets will patients be safe? If we meet the targets, we'll get new targets. It's a moveable thing and always will be. You know, hundred years ago we were wanting clean water..." (EDSRN5)

The balance of acute demand (ED shorter stays target) against "the business" (ICUSMO7, EDSRN1) of elective healthcare targets (elective surgery, faster cancer services) was frequently discussed. Clinicians indicated patient harm was likely when a "tipping point" (ICU SDR1) occurs somewhere in the system. Clinicians suggested organisational resources were frequently redistributed to meet a failing target, and this strategy created risk elsewhere.

"Sometimes it's a question of what's, I don't like the word target, but what Ministry directive are we going to, as an organisation, what trumps what?" (SEDRN1)

Managers suggested imbalance within the healthcare system influenced balance within the organisation. Participants suggested the risk of patient harm increased during periods of "flux" (EDSRN5) in operational activity. The disharmony created by flux led to organisational crowding and increased the risk of harm. Two antecedents contributed to flux; flow and surge. "Flow" (OM2, EDSRN 3, ICUSRN6) described the organisation's ability to move patients through the healthcare system. "Surge" (OM3, EDSRN1) depicted a large increase in demand at a given point in time. A rise in demand was attributed to increased acuity within the patient population or the number of patients attending ED - as seen in this comment describing the impact of surge:

"I find as one thing goes wrong, the next thing goes...one mechanism gets taken out, we're down a staff member for triage or there's 3 resus patients, so the ratio goes out there. We can handle 1, probably handle 2 but as the 3rd of the 4th come along that's when the risk increases." (EDSRN2)

Participants across roles and settings indicated systemic overcrowding increasingly influenced their ability to prevent harm. Organisational overcrowding was described as unsafe and was a regular feature in interviews and focus groups. ED patients in corridors, and poor ICU capacity were frequently discussed. ICU clinicians stated they often agreed to unplanned discharges, especially out of hours, or cancelled 
elective surgery to accommodate a 'sicker' patient. The impact of organisational overcrowding was multifaceted, and this comment was typical:

"The workload, the pressure, the distractions, the competing priorities, the demands... do this, do that, go here, notify that person, remember to do this, that can all (I call it a systemic problem,) contribute to getting it wrong." (OM3)

Imbalance was also created by conditions within the organisation's sphere of control (staff levels, elective surgery) and those outside of control (timing of MET calls, weather conditions). Clinicians had developed complex strategies to maintain patient safety at times of imbalance. For example, the decision to admit or discharge an individual patient to ICU required balancing multiple risks. Risks were considered regarding an individual patient's condition, the conditions of patients within the ICU and wider organisation, the consequences of delayed elective surgery and if patients with time-sensitive conditions could wait for retrieval from a rural hospital. Clinicians suggested imbalance increased the probability that decisions that were not ideal for one or multiple patients.

\subsection{Conclusion}

Findings suggest healthcare professionals understand and experience patient safety differently. The shared understanding of specific occupational groups influenced how healthcare professionals defined, approached and managed patient safety within the organisation. Therefore, how the phenomenon was framed, monitored, prioritised and communicated was different, and was influenced by occupational culture. The most divergent perspectives were between clinicians and managers. This is an important finding, as current definitions of safety culture require a shared understanding and commitment to patient safety systems. Therefore, the proposition that occupational cultures understand, experience and manage patient safety differently requires further discussion.

Healthcare professionals described harm as an inevitable part of the healthcare journey for some patients and agreed that preventable harm is unacceptable. Organisational safety capability determined the risk of harm, with clinicians stating managing clinical risk was part of everyday work. Clinicians described dynamic challenging conditions, caused by demand for acute care in which a number of 
predictable and unanticipated phenomena contributed to risk. In these conditions clinicians perceived current safety strategies and systems to be sub-optimal. The findings also indicate patient safety varies at any given point in time, and is to some degree dependent on luck in complex conditions. Given the centrality of these findings to the research question, and the importance and relevance of this to the propositions of this study, the next chapter will explore this in more depth. 


\section{Chapter 5: Discussion}

\subsection{Introduction}

This chapter will present and discuss findings using a structure that is guided by the propositions of the study. Table 4 aligns the relevant propositions alongside the key findings, presented according to the weight and saturation of the data. Study findings support the study propositions stated in Chapter 3 , and in doing so, add further evidence to current understanding of the phenomenon. In this chapter, contemporary literature is used triangulate and assist in the interpretation of the study findings. HDC decisions ${ }^{9}$ and national and organisational policies will be used to discuss the findings in the New Zealand healthcare context. Coroner and ACC cases were not mentioned by study participants, and as such have not been included. The resulting discussion describes findings in sufficient detail, maximising opportunities for propositional generalisation.

The most contrasting descriptions of safety culture were between (1) clinicians and managers, and (2) healthcare professionals working within the organisation, and their perceptions of the healthcare system. These differences will be described using Reason's (1997) terminology. The phrase 'sharp end' will be used to describe clinicians interacting with patients. Managers responsible for designing and enforcing the patient safety agenda within the organisation, and the wider healthcare system when this applies, are described as the 'blunt end'. Chapter 6 follows, concluding the research by examining the strengths and limitations of the study, and providing recommendations for patient safety within NZ acute care settings.

\footnotetext{
${ }^{9} \mathrm{HDC}$ cases will be referenced by website case number: http://www.hdc.org.nz/decisions--case-notes
} 
Table 4: Propositions related to study findings

\begin{tabular}{|c|c|}
\hline Proposition & Findings \\
\hline $\begin{array}{l}\text { 1. The majority of healthcare } \\
\text { professionals embrace the concept of } \\
\text { patient safety, with managers and } \\
\text { clinicians using different definitions } \\
\text { of patient safety. } \\
\text { Safety culture is dependent on the } \\
\text { actors in play, including the patient. } \\
\text { Therefore, safety culture is vulnerable, } \\
\text { and can be enhanced or derailed by } \\
\text { individuals and groups. There is a } \\
\text { negative stigma around harm and this } \\
\text { may contribute to a culture of safety in a } \\
\text { positive or negative way. }\end{array}$ & $\begin{array}{l}\text { Doctors, nurses and managers understand, } \\
\text { experience and manage patient safety } \\
\text { differently. These differences have created } \\
\text { conflict and some conditions that prevent } \\
\text { organisational resilience and recovery. } \\
\text { Healthcare professionals working at the } \\
\text { sharp end view current approaches as } \\
\text { reactive and too focused on the individual. } \\
\text { They believe this approach ignores factors } \\
\text { within the wider healthcare system and } \\
\text { have developed systems to account for } \\
\text { these factors. }\end{array}$ \\
\hline $\begin{array}{l}\text { 2. Patient safety in acute care has } \\
\text { unique challenges. } \\
\text { In these environments HRO approaches } \\
\text { focusing on rules and systems alone may } \\
\text { not be the best solution to improve safety } \\
\text { culture. Therefore, the measurement of } \\
\text { adverse incidents may not be a reliable } \\
\text { indicator of good safety culture. }\end{array}$ & $\begin{array}{l}\text { Safety capability influences patient safety. } \\
\text { Therefore, patient safety can alter at any } \\
\text { point in time. Patient safety requires a } \\
\text { vigilant and anticipatory approach to risk } \\
\text { and healthcare professionals have adopted } \\
\text { strategies to achieve this. Therefore, it is } \\
\text { unlikely that the adverse event reporting is } \\
\text { a reliable measurement of safety culture. }\end{array}$ \\
\hline $\begin{array}{l}\text { 3. Zero harm is unobtainable in } \\
\text { healthcare environments. } \\
\text { Therefore, harm may be better defined } \\
\text { as to 'do no iatrogenic harm'. There is a } \\
\text { point where harm reaches an acceptable } \\
\text { level, and acceptable harm may be } \\
\text { defined differently by the healthcare } \\
\text { professionals involved. }\end{array}$ & $\begin{array}{l}\text { Healthcare professionals describe patient } \\
\text { safety as reducing the risk of harm. Harm is } \\
\text { perceived to be an inevitable consequence } \\
\text { of healthcare for some patients. The risk of } \\
\text { exposure to harm is based on a complex } \\
\text { risk assessment process containing } \\
\text { objective and subjective data. How } \\
\text { healthcare professionals view risks to safety } \\
\text { is dependent on subjective judgments. }\end{array}$ \\
\hline
\end{tabular}




\subsection{Proposition 1: Healthcare professionals embrace the concept of patient safety. Occupational groups describe the phenomenon differently.}

\subsubsection{Keeping patients safe is a core value}

Members of all occupational groups held the view that patient safety was central to every decision. Prioritising patient safety in "everything we do" (EDSRN3) was discussed in relation to resource allocation, decisions about the clinical environment and complex treatment decisions. The value of patient safety was evident in organisational artefacts (e.g. EWS and clinical protocols), informal communication exemplars (e.g. bed meetings), and comments made in the interviews and focus groups (p. 71,74). The importance attributed to patient safety was illustrated in the following definition, provided by a senior clinician:

"(Patient safety is) about having the same message, the same understanding, the same brand, the same focus on what's important, and so it's trying to focus on what drives culture and leadership." (ICUSMO2)

This doctor is articulating the core message of the original definition of safety culture, that safety has to be the overwhelming shared priority of an organisation (e.g. INSAG ${ }^{10}$, 1991; Richter and Koch, 2004; Reason and Hobbs, 2003; Weick and Sutcliffe, 2001). Although the importance of patient safety was shared by doctors, nurses and managers, each occupational group had a unique approach to identifying and responding to risk. Managers used surveillance systems based on metrics, attended meetings, and relied on receiving reports from the clinicians. On the other hand, clinicians described how they used vigilance to anticipate and respond to clinical risk. This apparent contradiction was particularly evident in how the utilisation of patient safety strategies was described by participants from the blunt and sharp ends of the healthcare system.

The integration perspective (Martin, 2001) assumes organisations have a homogenous culture, dominated by easily identifiable management values. The integration perspective holds, like normative approaches, that culture is a variable

${ }^{10}$ International Nuclear Safety Advisory Group 
that can be measured, maintained and controlled. Others suggest culture is an entity that cannot be controlled (Geertz, 1973), and describe organisational culture as a complex sum of subcultures (Schein, 2004). Authors have identified that different perceptions of patient safety resulting from occupational culture do not reduce the incidence of harm (DOH, 2013; Waring, 2005). Findings from this study are consistent with this literature.

\subsubsection{Reactive versus proactive action}

Front line clinicians, including doctors, described the organisational incident reporting system as reactive (p. 67). This finding is unsurprising given the overwhelming body of literature stating doctors are not engaged with incident reporting systems (e.g. Pronovost et al., 2003; Robb and Seddon, 2010; Singer et al., 2003; Wagner, Smits, Sorra and Huang, 2013; Smits et al., 2012; Gawande, 2009; Waring, 2005). Conversely, managers were highly supportive of the incident reporting system stating it was "the bottom line" (OM3) for safety culture. This is an anticipated finding considering the unilateral adoption of this approach on an international scale $(\mathrm{WHO}$, 2009).

Managers valued safety systems that record events and respond using analysis to categorise and eliminate a root cause. This approach is consistent with NZ national policy (e.g. HQSC, 2015) and safety theory (e.g. Perrow,1984). Hollnagel et al. (2015) term this approach 'Safety l' where safety is defined as a "state where as few things as possible go wrong" (p.3). The sharp end valued proactive (p.67) systems characterised by vigilance and anticipation as beneficial for patient safety (e.g. EWS, CRM, re-triage). These tools are consistent with HRT (e.g. Sutcliffe 2011), and 'Safety II' (Hollnagel et al., 2015), as they attempt to control rather than eliminate hazards in complex conditions.

Managers described the incident reporting system as the primary surveillance tool for patient safety. In contrast, clinicians stated this system was useful to gain resources after rare events (p.67). Clinicians indicated the incident reporting system would not mitigate risk, stating "I can't say this (harm) is not going to happen again" (EDSDR10). 
The assertion that reporting a preventable adverse event does not avoid the identical event reoccurring is supported in HDC cases (e.g. 11HDCC01434,13HDC01676 ${ }^{11}$ ). Clinicians viewed the incident reporting system as bureaucratic (p.76). The validity and purpose of incident reporting systems as a strategy for improving patient safety is questioned (Banja, 2011; Leistikow, Mulder, Vesseur and Robben, 2016).

Furthermore, some theorists suggest a top-down rational approach of imposing rules limits freedom of choice, stifles innovation and feeds a culture of risk aversion and bureaucracy (Hale et al., 2013; Traynor, 2012). Theorists have suggested safety culture at the sharp end is not assured by "administration and ordering of the upstream system" (Dekker, 2015 p.vi), and generate safety bureaucracy described as:

“Undertaking an organizationally coordinated activity that, while legitimate with respect to organizational or societal goals (harm reduction, accident prevention), sustains demand for itself and creates more work to be met with additional bureaucratic means" (Dekker, 2014 p. 349)

Some scientists propose measurement is to be expected in a 'risk society' (e.g. Beck, 1992; Haukelid, 2008). Traynor (2012) suggests measurement in healthcare settings is a form of management surveillance to exercise power and control over healthcare professionals. Although there is no evidence of this in the study data, clinicians suggested incident reporting systems "find the one individual who the finger can be pointed at to make them stop doing it again" (ICUSDR3). This assertion is supported in the HQSC (2015) adverse event report, which notes DHB reviews focus on individuals, and fail to make suggestions about wider system issues. This point has also been raised by safety theorists (e.g. Dekker 2013, Vincent and Amalberti, 2016).

Clinicians indicated incident reporting systems create conflict between sharp and blunt ends of the healthcare system, and used language such as "tension" (ICUSDR2) and "battle" (EDSRN1), when describing interaction with managers. The conflict described suggests incident systems do not resonate with the intrinsic

\footnotetext{
${ }^{11} \mathrm{HDC}$ cases are referenced by website case number: http://www.hdc.org.nz/decisions--case-notes
} 
motivators of clinicians. This finding is important as theorists suggest intrinsic motivators are important if the intent is to control or shape culture, as

"the need for a sense of competence and personal determination, is derived from individuals' motivation to be the originators of their own behaviours rather than pawns to external forces." (O’Reilly and Chapman 1996 p.162)

The risk of harm occurring at the sharp end was reduced by vigilance and the application of protocols. Vigilant systems were described as triage and Early Warning Scoring (EWS) systems. International research findings, HDC case decisions and NZ policy suggest surveillance of a patient's clinical condition is warranted (NCEPOD, 2012; 13HDC00482, 05HDC11908). The HQSC (2016b) has recently launched a national deteriorating patient program to standardise an EWS across NZ, cementing the importance of vital sign surveillance in hospitals.

Protocols clinicians perceived to be valuable included fast track pathways for $\mathrm{PCI}^{12}$, which (Andersen and Terkelsen, 2006) estimate saves an extra 26 lives per 1,000 treated, when compared with existing treatment, and the ED shorter stays target. Managers indicated breaking protocols was unsafe (p.71). However, clinicians indicated breaking the rules was a daily occurrence, and was required to protect patients in unsafe conditions (p.70,71). Findings from other qualitative research studies identify clinical staff will work outside of organisational protocols that are not perceived to be relevant to an individual patient, or are inconsistent with their professional practice (Dixon-Woods et al., 2009; Drach-Zahavy and Somech, 2011; McDonald et al 2005; Waring, 2005). Management theorists agree, suggesting:

"Rigid bureaucratic rules can "help a company avoid fire fighting altogether, but at the price of almost no problems getting solved" (Bohn, 2000).

${ }^{12} \mathrm{PCI}$ Percutaneous Coronary Intervention 


\subsection{Proposition 2: Patient safety in acute care has unique challenges}

Patient safety issues in acute care are clearly complex, and situations may become unsafe at any time. Most NZ hospital events are acute admissions (51.6\%, Jiang and Pacheco, 2014), and increases in acute demand have been cited as a major challenge for both NZ EDs (Freeman and Parke, 2010), and ICUs (Corke, De Leeuw, Kai Lo and George, 2009). In these circumstances patient safety requires a vigilant and anticipatory approach to risk and healthcare professionals have adopted strategies to achieve this.

\subsubsection{Patient safety issues in acute care are complex}

Patient safety was not perceived to be a simple concept where care was either safe or unsafe, but instead patient safety was often described as occurring across different safety levels, where the risk of harm was dependent on the situation at any given time. Similarly, Vincent and Amalberti (2016) state "safety is a moving target" (p. 5), suggesting five levels of care exist in healthcare organisations, ranging from optimal benefit to an increased risk of harm. Findings from this study indicate different levels of care may also exist within one organisation at any given time.

Participants across the organisation recognised that safe delivery of acute care occurs within an interdependent and complex system. Correspondingly, 'interactive complexity' and 'coupling' are two key concepts described within normal accident theory, which is seminal patient safety work (i.e. NAT, Perrow, 1984). Interactive complexity describes the number and degree of system interrelationships; and 'tight coupling', is the degree to which initial failures can link together and have destructive impact on other parts of the system. Healthcare professionals in this study described safety capability as the interaction of a number of systems, actions and beliefs; this is congruent with high reliability theory (HRT). Organisations employing HRT believe safety is achieved by creating a workable survival that allows them to meet their goals, whilst acknowledging hazards remain (Reason, 2000; Weick and Sutcliffe, 2001). 
Healthcare professionals described conditions within the wider healthcare system that created imbalance and increased the risk of harm. Risks created by the blunt end of a system are termed latent conditions, whereas active failures are human errors and violations that produce immediate negative results (Reason, 1990). A significant task in healthcare professionals' day-to-day work was remaining vigilant to, and anticipating risks from both latent conditions and active failures. Clinicians described latent conditions culminated in a "tipping point" (ICUSDR1) where patient safety was compromised by competing elective and acute demand within a crowded system. ED crowding and ICU capacity problems caused by flow or surge created a 'perfect storm'13 where the risk of harm was certain within a "systemic problem (which) contributes to getting it wrong" (OM3). Systemic overcrowding and inadequate resources were identified as antecedents to rushed or untimely care by clinicians in ED (p.84) and ICU (p.85), who suggested similar conditions existed elsewhere within the organisation, especially when there were competing targets $(p$. 87). Negative descriptions of patient safety were particularly evident at times of systemic overcrowding, when "fire fighting" (ICUSDR5) behaviour increased across the organisation (p.70,73).

Ardagh and Drew (2015) estimate that crowding contributes to up to 300 deaths in NZ EDs annually. Crowding is a known antecedent to negative clinical outcomes in ED environments, including higher complication rates and mortality (Bergs et al., 2016). Harm from overcrowded emergency departments is well documented in international literature (e.g. Handel et al., 2010; Kallberg et al. 2015), and HDC decisions (07HDC17769, 07HDC10767, 08HDC00248, 07HDC14539). Hoot and Aronskys' (2008) systematic review of the causes, affects and solutions to ED crowding indicated patient harm occurs where there are latent conditions similar to those identified in this study (e.g. 68,83,84). The authors found a lack of resources (hospital beds, staff) as a major theme of the review.

Harm from crowding is not limited to ED environments, and EDs are often referred to as a "barometer" of healthcare system functionality (Coleman, 2014; O'Malley, Gerland, Pham and Berenson, 2005). A recent study by economists found a

\footnotetext{
${ }^{13} \mathrm{~A}$ perfect storm is a colloquialism denoting an especially bad situation caused by a combination of unfavourable circumstances.
} 
significantly elongated hospital stay in both elective and acute patient populations in NZ hospitals experiencing excess demand and concluded this may reflect quality of care issues (Jiang and Pacheco, 2014). Furthermore, Gabler et al. (2013) found patients admitted on days with ICU capacity strain experienced higher in-hospital mortality. These points are important as they indicate findings can be generalised beyond the case in question, particularly to organisations underperforming on the shorter stays healthcare target.

\subsubsection{Vigilance and anticipatory approaches to risk}

In order to manage such unpredictability, key vigilant and anticipatory approaches were used to recognise and respond to risk. These were evident at the sharp end (EWS, re-triage, informal communication) and the blunt end of the organisation (bed meetings, bed occupancy, TrendCare $\AA$ ). Clinicians used EWS and re-triage to remain vigilant to the unpredictable nature of patients, anticipating the human body can fail in acute illness. Managers used tools to remain vigilant to crowding and resource pressures. HROs also operate tools preoccupied with system failure, which focus on detecting and correcting threats to safety in real time:

"Organisational members try to anticipate and identify the events and occurrences that must not happen, identify all possible casual precursor events or conditions that may lead to them, and create a set of procedures for avoiding them." Sutcliffe (2011 p.136).

The finding that healthcare professionals have developed tools underpinned by the principles of high reliability theory supports a key assertion of Safety II theorists. Patient safety can be improved by understanding what healthcare professionals do well to reduce risk on the clinical shop floor (Hollnagel et al., 2015). Moreover, the presence of anticipatory and vigilant systems may be why there are so few adverse events given the inherent unreliability of the healthcare system. Systems like EWS and re-triage will support safer care delivery given Vincent's (2006) assertion that individual patients are unpredictable, and create risks impossible to mitigate using rule based approaches, such as protocols. Furthermore, safety culture requires creating workable patient safety solutions that will allow an organisation to achieve their goals, whilst acknowledging hazards remain (Weick and Sutcliffe, 2001; 
Hollnagel et a. 2015). All of these principles are evident in the anticipatory tools and systems healthcare professionals applied within the organisation.

There were different tools used to monitor and remain vigilant to patient safety at an organisational level, and a risk register was relied upon to monitor risk as "if you've still got a level of risk that's not tolerable, we have a responsibility to raise this to the board." (OM2). Senior managers oversaw a complex process before a hazard could be authorised to be added to the risk register. In NZ, clinical, financial and organisational risks are recorded in a model which emphasises considering risk in terms of the effect of uncertainty on objectives (Standards New Zealand, 2009). DHB managers are required to acknowledge patient safety risks within a prescribed matrix, alongside financial and reputation risks, including healthcare targets. Therefore, the tolerance of an identified risk, and the resulting risk assessment cannot be based on patient safety alone. HROs take a similar approach to risk management using risk registers to remain vigilant to, and anticipate the unexpected (Weick and Sutcliffe, 2001).

Risk models in HROs recognise they operate in uncertainty. Therefore, HROs are preoccupied with risks that occur at the sharp end of organisations (Reason, 2000; Sutcliffe, 2011). Conversely, the organisational approach to risk assessment was influenced by the fiscal climate (p.67,69), hospital board tolerance to financial risk, and political/reputational considerations within the public healthcare system (p.76). Decisions of the likelihood of risk causing harm were made in meetings which did not include expertise from the sharp end of acute care. This is an important point as authors have found the occupational groups classify risk differently (Dixon-Woods et al., 2009; Waring, 2005), and risks are downgraded by NZ managers (Hardy, 2013). Safety scientists suggest that deference to expertise is an important facet of HRO safety culture (e.g. Sutcliffe 2011). Decisions about safety are delegated to the 'expert' with the skills and knowledge to inform decision making regarding a risk, rather than being defined by traditional hierarchical structures. The requirement to consider patient safety risks alongside other organisational risks may explain the inconsistency between managers' comments that patient safety was the overriding priority of the organisation, and perceptions at the sharp end that the managers' approach to safety was tantamount to "lip service" (EDSMO10). 
Clinicians viewed harm resulting from reported hazards as unacceptable if they had been reported as near misses (p.66), or resource requests had been declined. suggesting "it's easier to get resources to fix a rare problem, that has caused a single bad event than introduce a concept of improvement" (ICUSMO2). Near misses are common in healthcare environments, occurring up to 300 times more frequently than adverse events (Wolf and Hughes, 2008). A focus on near misses, or small repetitive events, is congruent with the 'mindful' approach in HROs where learning from previous, or potential failures as a proactive prevention strategy (Reason, 2000; Weick and Sutcliffe, 2007). Indeed, there is increasing recognition that harm from cumulative minor failures is greater than harm from significant adverse events in healthcare environments (Barach and Small 2000; Hollnagel et al., 2015; Vincent and Amalberti, 2016). Authors suggest learning from the root cause analysis of adverse events is outdated in increasingly complicated and intractable socio-technical systems (Hollnagel, 2015), and others, including Reason himself, suggest Reason's (1997) famous 'Swiss cheese model' is outdated (Reason, Hollnagel \& Paries, 2006).

Managers indicated they were interested in near miss reporting whereas clinicians reported a patient safety culture tolerant of those risk and hazards with unclear ownership (p.77) lacking feedback (p.66). Reason (1998) suggests distance from the sharp end makes it "easy not to be afraid" (p. 296) of hazards that endanger operations. The consequence of failure to remove hazards was the development of fire fighting behaviours which will be explored further. This is important as fire fighting can have unintended consequences for patient safety (Perry, Wears and Fairbanks, 2012).

\subsubsection{Fire fighting: a strategy to manage risk}

Clinicians at the sharp end suggested traditional strategies, such as protocols, cannot be relied upon exclusively to provide safe care in acute healthcare environments. One doctor stated:

"I hate the comparison between airlines and emergency medicine because a pilot would never climb on a plane that is not good to fly, yet all our planes are crook." (EDSDR5). 
Over a decade ago Berwick (2002) observed that healthcare environments differ from traditional industries, and more recently Hollnagel et al. (2015) proposed implementation of strategies from traditional industries into healthcare without this consideration is regrettable. Authors agree that patients bring an unpredictability to acute healthcare environments that is impossible to control using traditional safety systems alone (Vincent, 2006; Gawande, 2009; Perry, Wears and Fairbanks, 2012). At times of system imbalance clinicians suggested fire fighting reduced clinical risk and respond to anticipated and unanticipated events within the "background of chaos" (EDSRN2) by "strategizing around situations" (ICUSRN5). Clinicians described providing responsive and safe patient care where there are unanticipated delays, or they were unable to provide timely or efficient care by "fighting fires" (ICUSDR5).

Fire fighting skills were highly valued improvisation skills that clinicians indicated were gained from experience and exposure to risky situations, and had become part of normal everyday work. Managers also described fire fighting behaviours using tools to predict and control limited resources (e.g. TrendCare $\AA$, bed occupancy) around the organisation. High reliability theorists suggest "ambivalence builds resilience" in organisations (Weick and Sutcliffe, 2001, p. 167), and suggest these types of behaviours are required to mitigate immediate risk at the sharp end of operations (Hollnagel et al., 2015; Dixon Woods, 2009; Reason, 2000), and build organisational resilience (Weick and Sutcliffe, 2001).

Bohn (2000) proposes organisational fire fighting has a collection of behaviours, many of which are evident in the study findings including; a lack of time to solve problems, incomplete solutions, reoccurring and cascading problems, urgency superseding importance, problems become crisis and decreased performance. Authors caution against overdoing lean ideals that remove the capacity for resilience and flexibility (Weick and Sutcliffe, 2001). Similarly, clinicians in this study described essential building blocks are required to create a "buffer" (ICUDR2) for safe care delivery. Although fire fighting was described as a positive and desirable characteristic by study participants, fire fighting can have unintended consequences for patient safety when it becomes a facet of organisational culture (e.g. DOH, 2013). Authors identify a constant interplay between quality and efficiency exists in acute care environments (Perry, Wears and Fairbanks, 2012; Nugus and Braithwaite, 
2010), and imbalance between these characteristics leads to "care rationing" (Nugus and Braithwaite, 2010, p. 512).

Fire fighting requires appropriate resources are available, and is vulnerable. Clinicians indicated disturbances in appropriate resources increases the risk of errors and harm. Fire fighting skills were held in high regard (p.71,74) and were acquired in a similar manner to technical clinical skills, through clinical exposure to risk, repetition and work arounds. Fire fighting, as a proactive strategy, was viewed as a skill held by leaders within the organisation (p.74), particularly during crowding. Safety theorists suggest when the aspiration is to deliver patient care that is "good enough" (p. 21), patients will be exposed to variation, poor standards and hazards (Vincent and Amalberti, 2016).

Safety theorists call fire fighting behaviour 'patching', a term originating from engineering (Perry, Wears and Fairbanks, 2012). Patching can encourage "muddling through" behaviour with unintended consequences for patient safety, by encouraging unwanted variation (Perry, Wears and Fairbanks, 2012, p. 716). Unwarranted variation in healthcare is any variation with a harmful consequence (Appleby and Raleigh 2011). In this study patching behaviour was reported to impact on patient safety. This was evident in ED, where patients were re-triaged and treated in corridors, a recognised antecedent to harm monitored by the ED shorter stay target in NZ (Ardagh, 2009; Ardagh and Drew, 2015; Bergs et al. 2016; Handel et al., 2010).

Clinicians described factoring organisational context into their decision making when there were several patients competing for ICU beds (p.84), suggesting this affected patient safety, especially when required to discharge patients at night, or to meet acute demand and elective surgery targets (p.85). Studies of large databases identified discharging patients from ICU at night significantly increases in-hospital mortality (Laupland et al., 2011; Osborne, 2007), and could be explained by decreased night-time resources (Osbourne, 2007). Similarly, organisational rosters in this study also demonstrated night-time and weekend resources are reduced, especially for senior staff (Appendix 8). 


\subsubsection{Developing organisational resilience}

Findings from this study indicate building blocks, collaboration and trust, and balance contribute to resilience within healthcare organisations. Organisational theorists always approach resilience as a "positive and desirable" characteristic (Mamouni, Liminos, Mazzarol, Ghadouani and Schizzi, 2014, p.106). In this study participant's descriptions of resilience were congruent with Hollnagel's definition:

"The essence of resilience is the intrinsic ability of a system to adjust its functioning prior to, during, or following changes and disturbances, so that it can sustain required operations under both expected and unexpected conditions." (Hollnagel, Pariès, Woods and Wreathall, 2010).

EWS and triage systems contributed to a resilient culture by evoking a strong, prescribed response and mobilising clinicians with the expertise to manage a situation. Safety leadership at the sharp end was provided by "go to people" (EDRN4), who were trusted to provide direction and support, especially when standard rules were not applicable. Two recent systematic reviews support the importance of "go to people" for patient safety, finding a positive correlation between patient outcomes and supervision of junior medical and nursing staff (Van der Leeuw, Lombarts, Arah, Heineman, 2012; Stalpers, de Brouwer, Kaljouw and Schuurmans, 2015).

Theorists also recommend reconfiguring leadership to conditions (Weick and Sutcliffe, 2001). In routine mode, HROs are controlled in the conventional hierarchical manner, but in high tempo situations control shifts to the experts on the spot (Reason, 2000). This leadership structure is highly regarded and is known as 'deference to expertise' (Wieck and Sutcliffe, 2001; Reason, 2000). A correlation between flat hierarchy and patient safety is a finding in contemporary safety research (Armellino, Quinn Griffin, Fitzpatrick, 2010; Gawande, 2009; Reader, Flin and Cuthbertson, 2007; Sexton et al., 2011), and literature that promotes the empowerment of nursing staff (Shine, 2013; Keough, 2013).

In contrast, participants indicated the structure of their work environment reduced safety capability, and provided examples where they were unable to provide safe care (p.68,69,84). A lack of empowerment to create safety changes was most 
frequently described by clinicians at the sharp end (p.67,69) and often resulted in conflict with managers (p.79). Kanter (1993) proposes the structure of the work environment correlates to employee's attitudes and behaviours, and safety culture has long been defined as an "assembly of characteristics and attitudes in organizations and individuals which establishes that, as an overriding priority, safety issues receive the attention warranted by their significance." (INSAG, 1991 p.14). These findings suggest patient safety requires structures that enable decisions based on skill level and expertise, rather than organisational position. This will require healthcare organisations to give the sharp end the authority to make decisions about risk and the resources to implement them. This is likely to make significant gains in patient safety culture.

Clinicians indicated collaboration is required to keep patients safe. Descriptions of collaboration were underpinned by informal power structures that came from building relationships and alliances with peers and colleagues and contributed to good teamwork $(p .80,81)$. A recent systematic review concludes that collaboration it is of the utmost importance to improve communication with patients and between professionals in order to maintain and enhance safety (Severinsson, Haruna, Rönnerhag, Berggren, 2015). Healthcare professionals stressed the importance of informal communication in day-to-day work, suggesting it plays a vital role in collaboration and provides the basis of risk assessments to prevent adverse events within complex conditions. Reason (2000) identifies a collective preoccupation with the possibility of failure as most important distinguishing feature of high reliability organisations. HROs recognise that:

"human variability in the shape of compensations and adaptations to changing events represents one of the system's most important safeguards" (Reason, $2000 \mathrm{p}$. 769)

The capability to manage risk through informal communications, is a characteristic of HROs (Sutcliffe, 2011). A common language to communicate risk was provided by the safety tools and systems healthcare professionals described (e.g. EWS, retriage, TrendCare $\AA^{\circledR}$. Clinicians also indicated risk was managed through constant informal conversations such as meetings, huddles or telephone calls. Informal communication cannot be measured, manipulated or controlled and adds weight to 
the proposal that healthcare safety culture cannot be measured solely using adverse incident reporting. Retrospective analysis of adverse events will not account for this element of a resilient culture.

HROs apply the principle of mindful organisation, which requires respectful interaction to be the norm, so employees are aware of how their work fits with others and within organisational goals (Sutcliffe, 2011). Furthermore, HROs stress safety culture requires an appreciation of the importance of interfaces, interconnections, and interdependence between and among system elements (Grabowski and Roberts, 1997). Westrum's (1993) theory of generative culture also requires all workers in an organisation make concerns about safety known. This literature explains why participants described poor behaviour (p.80) as negatively impacting on patient safety. Considering the importance of collaboration, a deeper understanding of why refusal of ED referrals was described as a cultural problem (p.81). This finding suggests forcing functions, such as the ED shorter stays health target, will not prove to be successful, unless barriers to collaboration are addressed.

\subsection{Proposition 3: Zero harm is unobtainable in healthcare environments}

Healthcare professionals described reducing the risk or consequence of harm as the inevitable outcome of patient safety. Risk is defined as the probability that an adverse event will occur within a specific time period, or as a result of a specific situation (NPSA, 2008 p. 4). From this, a hazard is defined as anything that has the potential to cause harm, a risk assessment is required for each identified hazard based on objective and subjective data. Within organisations, decisions are made regarding the significance of the risk, whether appropriate controls or contingencies are in place.

\subsubsection{Patient safety as reducing the risk of harm}

Healthcare professional's descriptions of patient safety support the current WHO (2009) definition. Findings from this study support the view that practicing clinicians, are willing to discuss reducing the risk of preventable harm (Pronovost and Colantuoni, 2009; Gawande, 2009; Bagnara et al., 2010). This position is congruent with international healthcare practice that seeks to eliminate risk as far as is 
reasonable and practicable (NPSA, 2008), and normal accident theory (Perrow, 1984). Hollnagel et al. (2015) suggest defining safety by the success of harm prevention (Safety I), is the inevitable consequence of measuring patient safety by measuring patient harm. Findings indicate that strategies intending to eliminate harm using Safety I strategies alone will not be successful.

Participants described harm that was caused by processes of care intended to diagnose or treat patients, and conditions within the healthcare system; this will be referred to as iatrogenic harm. Study participants identified a number of hazards contribute to iatrogenic harm and these are similarly discussed in the literature. The most prolific hazards identified by both groups include increasing co-morbidity in patient populations, increasing number and complexity of treatment options and subspecialisation (Gawande, 2009, 2014; Berwick, 2016; Carayon, 2012; Vincent and Amalberti, 2016).

Healthcare organisations differ from traditional HROs such as the aviation industry, where safety improvements over the past fifteen years have been attributed to increased engine performance and reliability and automatic warning mechanisms (Oster, Strong and Zorn, 2013). Therefore, the comparison of safety records of aviation and healthcare organisations will continue to differ. Unlike the aviation industry where challenges remain static, the complexity of conditions and demand in the healthcare environment ensures the risk reduction is more challenging.

\subsubsection{Harm as an inevitable consequence}

Healthcare professionals understood and identified iatrogenic harm as an inevitable consequence of intervention for some patients. Clinicians identified several hazards to patient safety that had been reported but not addressed, and identified the potential for harm remained (p. 67). A contradictory relationship between patient safety, and financial and reputational responsibility, was described by multiple participants. Clinicians perceived the organisational focus was on fiscal responsibility, which had a detrimental effect on patient safety. This perspective was discussed to a lesser extent by managers, though field notes indicate they held similar views. One manager stated "There are minimum standards, and there are gold plate standards, and we can't afford gold plate." (OM2). Hardy's (2013) study 
concluded that managers' ability to proactively plan safe care is limited by annual financial constraints in NZ DHB funding. Contrastingly, the IHI (2016) suggest senior leaders must demonstrate a commitment to safety and provide the resources to achieve results if they want a culture of safety. HROs have also struggled to prioritise safety, and Ed Schein suggests poor safety culture in nuclear power can be directly attributed to leaders focusing on finance over safety:

"Senior executives care more about finances than safety, middle managers care more about productivity because that is what senior managers reward them for, and supervisors suppress employee complaints and efforts to identify safety problems because it takes too much time to look into things and to convince their bosses about critical maintenance issues that may be surfacing." (Schein cited Collins, 2010, p. 10)

Findings from this data indicate the NZ government does not grasp the investment required for patient safety on the front line. Alternatively, policy makers are distant from the sharp end and this may influence their risk perception or understanding of conditions at the front line (Reason, 1997; EDRN4, p.75). Field notes indicate managers perceived speaking publicly about the relationship between safety and finance would not be acceptable, indicating policy makers may not be aware of the stories Berwick (2002) ${ }^{14}$ suggests are necessary to gain knowledge about safety. Overreliance on measurement and targets as indicators of positive safety culture, may also contribute to this picture. Keene et al (2016) suggest the NZ government can and should spend more on health. New Zealand has a modest level of health spending overall, with government health spending decreasing as a proportion of GDP (Keene et al., 2016). Conversely, many western countries have the opposite trend, where healthcare is typically the largest single component of GDP (The European Centre for International Political Economy, 2011).

\subsubsection{Risk assessment processes to mitigate iatrogenic harm}

The risk of harm was perceived as tolerable if an action intended to reduce the morbidity and/or mortality risk for a patient suffering or dying, as long as harm was minimised with appropriate informed consent and quality control measures. This

\footnotetext{
${ }^{14}$ Page ix of this thesis, lessons from a novice
} 
philosophy is common in healthcare and is generally accepted as congruent with the principle of 'primum non nocere' (first do no harm). The World Medical Association (WMA, 2015) suggests to 'do no harm' requires a balance between the underlying principles of non-maleficence and beneficence in clinical practice. In simple terms this implies it is acceptable to inflict the least harm possible to reach a beneficial outcome for a patient. For example, advances in surgery have been made possible by the experimental culture of medicine (Gawande, 2009).

Front line clinicians indicated that exposure to harm changes risk perception. The perception that harm is inevitable was less prevalent in junior (RNs, Registrars) than senior clinicians (consultants, senior nurses, managers). In the ICU doctors focus group, consideration was given as to whether junior staff were idealistic about patient safety. A heightened awareness of the risks associated with medical procedures is gained through medical training and experience (Braithwaite, Westbrook, Travaglia and Hughes, 2010). Clinicians in this study described using "risk stratification" (EDSDR5) to deliver safe patient care in every day practice.

Risk stratification applied the benefit-risk ratio, a complex process that considers the seriousness of a problem, the efficacy and safety of treatment proposed, and the efficacy and safety of other options available (Aronson and Ferner, 2005). The benefit-risk ratio is an important concept in clinical decision-making, determining a course of action by considering positive potential outcomes for patients, compared to the risk of harm. Aronson and Ferner (2005) suggest benefits and risks are noncomparable, stating "benefit is an actual outcome, while a risk is a chance of an outcome" (p. 867). Therefore, beneficial outcomes, just like adverse events can only be determined in retrospect. In emergency situations, such as MET calls and in ED resuscitation, clinicians often had minimal information, and were required to adapt and respond. Clinical staff placed value on learning from extreme events with positive and negative outcomes, and skills gained through exposure to risk. This approach is supported by a number of authors (Carayon, 2012; Flannagan, 1954; Hollnagel et al., 2015).

Clinicians suggested patients must be empowered and informed partners in the risk assessment process, describing the patients "personal approach to risk" (ICUDR2). This view, that patient safety is associated with patient participation, is congruent with 
theoretical work (e.g. Gawande, 2014) and research indicates active patient participation reduces medical errors (Awe and Lin, 2003; Blendon, DesRoches and Cohen, 1999; Davis, Jacklin, Sevdalis and Vincent 2007). Furthermore, the importance of co-designing care is also stressed by safety scientists (e.g. Gawande, 2014) and the quality improvement movement (e.g. HQSC, 2016c). Clinicians described harm that resulted from treatment that was not of value to individual patients. Literature suggests treatment that has a "lack of benefit" is unnecessary, and can be harmful (Aronson, 2009, p. 601).

Harm from over treatment is documented in many healthcare settings (Cohen, 1999; Mitka, 2008; Diamandis, 2015; Biller-Andorno and Juni, 2014; Gawande, 2014). Moreover, the literature suggests clinicians' concern about the personal approach to risk is warranted, for example one third of NZ palliative care patients will visit the ED at some stage of their illness (Lawrenson et al., 2012). Similarly, although most NZ hospitals require goals of treatment to be documented within 24 hours of an acute admission, approximately one third of hospital MET calls conclude in a treatment limitation plan (HQSC, 2016b). This may explain the value ICU clinicians attributed to the MET team, as early decision making intervenes to prevent harm from 'over intervention' often whilst the patient is still able to talk. The importance of the personal approach to risk is felt to be of such importance the NZ ED chair of the Australasian College of Emergency Physicians recently requested non-completion of advanced care plans is classed as an adverse event (Bonning and Lawler cited HQSC, 2016). Therefore, study findings suggest the current focus on understanding a patient's wishes and preferences is of vital importance (e.g. MH, 2011; HQSC, 2016b). However, findings also suggest our understanding of patients' risk perception is currently limited to what clinicians and policy makers believe is important, and this concept may require further investigation.

Clinicians and managers viewed staff burnout as a preventable harmful outcome of working in acute care environments. Healthcare professionals in this study described prevention of "burnout" (OM1) as an important facet of safety leadership in a resilient culture. A different tolerance to this hazard was evident within the organisation; clinical staff described burnout as a characteristic of their current environment, where there was no opportunity for recovery (p. 75). Managers indicated burnout point had not been reached (p. 75). This is an important finding as safety culture is affected in 
environments where staff do not feel cared for (IOM, 2015). Several authors have identified moral distress attributed to patient harm by nursing staff in acute care including situations where patients are not expected to benefit from aggressive care (Elpern, Covert and Kleinpell, 2005; Zuzelo, 2007), unnecessary tests and treatments (Zuzelo, 2007) and where harm is attributed to unsafe staffing levels (Aitken et al., 2014; Francis, 2013; Lankshear et al. 2005; Rafferty et al 2007; Royal College of Nurses (RCN), 2008). Moral distress is also evident in the report into Mid Staffordshire Hospital, where many healthcare professionals expressed profound regret for normalising poor patient care $(\mathrm{DH}, 2013)$. This finding suggests preventable harm, patient safety and staff welfare are interrelated.

\subsection{Conclusion}

This chapter has brought together findings from this case study and, when synthesised with the literature, brings a more detailed understanding of patient safety in acute care hospitals. By drawing attention to the similarities and differences across clinical areas, and occupational groups, a greater appreciation of how clinicians and managers work with, and strategize to address the patient safety agenda has been realised. The contribution of this study to practice, knowledge, and policy is now further explored in Chapter 6. 


\section{Chapter 6: Conclusion}

\subsection{Introduction}

This thesis presents important knowledge about how patient safety is understood and experienced by healthcare professionals using qualitative methodology and methods. Exploration of healthcare professionals' subjective experiences of patient safety has identified contrasting perceptions and understandings, especially between those working at the blunt and sharp ends of the healthcare system. This chapter will systematically explore the implications, strengths and limitations of this study, so that new knowledge can be developed further. The chapter concludes with recommendations for future research.

\subsection{Implications of the study}

A significant body of research has explored health professionals' understanding of patient safety. Predominantly using surveys has provided inconsistent results. Poor overall survey response rates, particularly from doctors, has led researchers and regulators to question if healthcare professionals are engaged in the patient safety movement.

Findings from this case study are inconsistent with this proposition, revealing patient safety is an acknowledged and valued aspect of health professional responsibilities, but is perceived and described differently by individual professional groups. The study identifies healthcare professionals working at the sharp and blunt end of healthcare hold patient safety as a core value at the heart of their work, although patient safety was understood or perceived from different perspectives. The research findings suggest patient safety is compromised as these differences conflict. The study has implications for the concept of patient safety and the capability of delivering safe patient care. The following section will examine these implications in detail. 


\subsection{Anticipation and Vigilance}

Acute hospitals are unlikely to make significant gains in patient safety solely through reliance on protocols or procedures. Healthcare professionals recognise that proactive systems, underpinned by anticipation and vigilance are required, and contribute to safe care. The value of these systems is currently underestimated by managers and NZ healthcare policy. Furthermore, a paradigm shift from a reactive system that values recognising and reporting harm (Safety I) to a proactive system that also values safe outcomes and culture (Safety II) is required. Study findings indicate this approach is possible and likely to be embraced by clinicians, who continue to be disengaged with what are perceived as reactive and punitive reporting methods. Clinicians indicated they are overwhelmed with the current pace of change and competing priorities. The level of change required to develop a successful patient safety culture and engage clinicians suggests that a timely approach that prioritises safety over efficiency is required.

\subsection{Resilient Culture}

Organisations require a buffer to effectively recover, reflect and respond to safety incidents and concerns. Healthcare professionals identified that a resilient culture provides a dynamic yet stable state during periods of imbalance or unanticipated conditions when organisations are at their most vulnerable. In this study, resilient culture was underpinned by key resource building blocks, collaboration and trust and balance, all of which require investment in resources and human relationships.

Healthcare professionals indicated they had limited control over safe care delivery, especially at the sharp end of the organisation. The study demonstrates healthcare organisations will need to consider flexible power structures that defer to expertise at the sharp end. Managers who demonstrate trust in clinicians to deliver safe patient care are likely to create opportunities for collaboration. This study demonstrates that if policy makers and managers require everyone to own safety, they must be prepared to distribute leadership and devolve responsibility. 


\subsection{Strengths and Limitations of the research}

A strength of this thesis was the choice of embedded case study. Use of multiple sources of data (interviews, focus groups) from multiple cases allowed rich detail to explore a multifaceted research problem. Furthermore, case study design enabled a detailed exploration of the context in which patent safety occurs, including the triangulation with research literature and health policy. This enabled exploration at both the sharp and blunt ends of healthcare thereby strengthening methodological rigour and offering the opportunity to make analytical generalisations beyond the case in question.

A second strength of this study is that field work was undertaken within the clinical environment by a researcher cognisant of health care and its challenges, whilst being reflexive to the challenges of insider research. In building a rapport with participants and spending time in the field, as recommended by proponents of case study research, healthcare staff were able to describe their daily practice in an open way. Most gatekeepers were proactive and supportive of the research and ED medical staff requested an additional focus group session to describe and discuss their understanding and concerns. The engagement of doctors in this study, a further study strength, contrasts with the response rates from this occupational group to quantitative survey research.

The small number of organisational managers in the sample has allowed the views of the clinicians to predominate the data. Therefore, an imbalance of participants at the sharp and blunt ends of healthcare limits interpretation of managers' perceptions of patient safety. This was recognised as a limitation and attempts were made to generalise findings from the blunt end of the wider healthcare system using policy and the literature. To attend to the critique that findings from this study are only relevant to healthcare professionals within the case in question, replication of this study would be advantageous. 


\subsection{Recommendations for further research}

Patient safety is a multifaceted phenomenon influenced by complex factors. Several questions and issues have evolved that require further exploration.

1. Measurement of patient safety culture may not be possible using quantitative methodology. As demonstrated in this study, researchers may be better able to understand safety culture using qualitative methodology and methods. This may be undertaken through use of case study or ethnographic exploration.

2. Measurement of adverse incidents is not a good indicator of safety culture. However, retrospective or prospective review of these databases could provide data used to develop anticipatory and vigilant systems for safe care delivery.

3. Findings in this study are limited to acute care environments. However, the divergence of opinion between those at the sharp and blunt ends of healthcare requires further exploration in other settings. The study would benefit from replication in other non-specialised areas, for example general medicine, which have different workflow to identify common and divergent factors that contribute to patient safety culture.

4. Although the relationship between patient safety and ED crowding is widely discussed in the literature, there is a limited understanding of the impact of system crowding on patient safety. Research that identifies healthcare professionals' experience and management strategies of health system crowding may identify new insights.

5. The current study found that patient safety is dependent on collaboration between occupational groups, yet many relationships are characterised by conflict. Furthermore, forcing functions attempting to improve collaboration do not appear to have made significant gains in safety capability. Research that considers the barriers and enablers for collaboration may provide new insights. 


\subsection{Conclusion}

The aim of this thesis was to describe how healthcare professionals in a NZ tertiary hospital described patient safety. The embedded case study demonstrated patient safety is not uniformly understood nor managed across professional groups within the intricate environment of an acute care hospital. Given that patient safety is perceived to exist across different levels of safety, healthcare professionals accept that some harm is inevitable in the healthcare setting. Doctors, nurses and managers understand and manage patient safety differently, and this affects how patient safety is addressed, through reactive incident reporting systems to proactive systems of vigilance.

To keep patients safe and avoid harm, more proactive patient safety systems are needed to manage patient safety in hospitals. This will require a paradigm shift away from current reactive to proactive safety systems to bring flexibility and stability to the complex acute care environments. 


\section{References}

Agency for Healthcare Research and Quality. (2013). Eliminating CLABSI, A National Patient Safety Imperative: Final Report Companion Guide. Retrieved from http://www.ahrq.gov/professionals/quality-patient-safety/cusp/clabsi-finalcompanion/clabsicomp5a.html

Agency for Healthcare Research and Quality. (2015). Human Factors Engineering. Retrieved from https://psnet.ahrq.gov/primers/primer/20/human-factors-engineering Agency for Healthcare Research and Quality. (2014). Voluntary Patient Safety Event Reporting. Retrieved from https://psnet.ahrq.gov/primers/primer/13/voluntary-patientsafety-event-reporting-incident-reporting

Alahmadi, H. (2010). Assessment of patient safety culture in Saudi Arabian hospitals. Quality Safety Health Care, 19, 1-5 doi:10.1136/qshc.2009.033258

Allan, D., Judith, S. (2005). Review of the Australian incident monitoring system. Australia and New Zealand Journal of Surgery, 75, 8, 657-71.

Andersen, H., Terkelsen, C. (2006). Fast-track primary percutaneous coronary intervention in patients with ST-Elevation myocardial infarction. Interventional Cardiology Review, 11 (2) Retrieved from https://www.radcliffecardiology.com/articles/fast-track-primary-percutaneouscoronary-intervention-patients-st-elevation-myocardial

Appleby, J. Raleigh, V (2011) Variations in healthcare - the good, the bad and the inexplicable. Retrieved from http://www.kingsfund.org.uk/publications/helathcare_varaition.html.

Al-Sawa, A. (2013). Leadership of Healthcare Professionals: Where Do We Stand? Oman Medical Journal, 28, 4, 285-287.

Allsop, J. (2006). Medical dominance in a changing world: the UK case. Health Sociology Review, 15, 5, 444-457. 
Amalberti, R., Auroy, Y., Berwick, D., Barach, P. (2005). Five System Barriers to Achieving Ultra Safe Healthcare. Annuals of Internal Medicine, 142, 756-764.

Ardagh, M. (2009). What do we do about increasing demand for emergency department care? The New Zealand Medical Journal, 122, 1302, 9-10.

Ardagh, M., Drew, L. (2015). What have five years of the shorter stays in ED health target done to us? The New Zealand Medical Journal, 218, 1421.

Armellino D., Quinn., Griffin, M., Fitzpatrick, J. (2010). Structural empowerment and patient safety culture among registered nurses working in adult critical care units. Journal of Nursing Management, 18, 7, 796-803.

Aronson, J., Ferner, R. (2005). Clarification of Terminology in Drug Safety. Drug Safety, 28, 10, 851-870

Aronson, J. (2009). Medication errors: Definitions and classifications. British Journal of Clinical Pharmacology, 67, 6, 599-604.

Ashcroft, D., Morecroft, C., Parker, D., Noyce, P. (2005). Safety Culture assessment in community pharmacy: development, face validity, and feasibility of the Manchester Patient Safety Assessment Framework. Quality Safety Healthcare 14, 417-42.

Atkin, L. (2014). Nurse Cuts Linked to Death rates. Nursing Times Online. Retrieved from https://www.nursingtimes.net/clinical-archive/patient-safety/nurse-cuts-linked-todeath-rates-says-major-study/5068387.article

Australian and New Zealand Intensive Care Society. (2015). Annual report. ANZICS. Carlton, Australia.

Awe, C., Lin, S. (2003). A patient empowerment model to prevent medication errors. Journal of Medical Systems 27, 6, 503-17.

Ayres, L., Kavanaugh, K., Knafl, K. (2003). Within-case and across-case approaches to qualitative data analysis. Qualitative Health Research, 13, 6, 871-883.

Bagnara, S., Parlangeli, O., Tartaglia, R. (2010). Are hospitals becoming high reliability organisations? Applied Ergonomics, 41, 713-718. 
Baker, G. (2012). The challenges of making healthcare safer: Leadership and system transformation. Healthcare Quarterly, 15, 8-11.

Banja, J. (2011). The normalization of deviance in healthcare delivery. Retrieved from http://www.ncbi.nlm.nih.gov/pmc/articles/PMC2821100/

Barach. P., Small, S. (2000). Reporting and preventing medical mishaps: lessons from non-medical near miss reporting systems. British Medical Journal 320, 7237, 759-63.

Baxter, P., Jack, S. (2008). Qualitative Case Study Methodology: Study Design and Implementation for Novice Researchers. The Qualitative Report, 13, 4, 544-559.

Beck, U. (1992). Risk society towards a new modernity. Newbury Park, US: Sage Publications

Berghan, G. (2007). What does a collective identity mean from a Maori point of view? How would the collective identity fit with a Maori worldview and the realities of Maori health promotion? Retrieved from http://www.hauora.co.nz/resources/Maoripointofview.pdf

Bergs, J., Vandijck, D., Hoogmartens, O., Heerinck, P., Van Sassenbroeck, D., Depaire, B., Marneffe, W., Verelst, S. (2016). Emergency department crowding: Time to shift the paradigm from predicting and controlling to analysing and managing. International Emergency Nursing, 24, 74-77.

Berwick, D. (2016). Era 3 for medicine and health care. Journal of the American Medical Association, 315, 12 retrieved from http://www.ihi.org/resources/Pages/Publications/Era-Three-for-Medicine-HealthCare.aspx

Berwick, D. (2002). Patient safety: Lessons from a novice. Advances in Neonatal Care, 2, 3, 121-2. 
Bion, J., Richardson, A., Hibbert P., Beer, J., Abrusci, T., McCutcheon, M., Cassidy, J., Eddleston, J., Gunning, K., Bellingan, G., Patten, M., Harrison, D., and the Matching Michigan writing committee. (2012). Matching Michigan': a 2-year stepped interventional programme to minimise central venous catheter-blood stream infections in intensive care units in England. British Medical Journal of Quality and Safety, 22, 2, 110-23.

Blendon, R., DesRoches, C., Brodie, M., Benson, J., Rosen, A., Schneider, E., Altman, D., Zapert, K., Herrmann, M., Steffenson, A. (2002). Views of Practicing Physicians and the Public on Medical Errors. New England Journal of Medicine, 347 24, 1933-1940.

Bodur, S., Filiz, E. (2009). A survey on patient safety culture in primary healthcare services in Turkey. International journal for Quality in Health Care, 21, 5 348-355.

Bohn, R. (2000). Stop Fighting fires. Harvard business review online. Retrieved from https://hbr.org/2000/07/stop-fighting-fires

Bonning, J., Lawler, A. (2016). Formal response to the HQSC paper: The deteriorating patient, current practice and emerging themes discussion paper in HQSC: The deteriorating adult patient. Current practice and emerging themes. HQSC, Wellington. NZ

Borkan, J. (1999). "Immersion/Crystallization." In Crabtree B, Miller. W. (Eds.) Doing Qualitative Research (2nd Edition). Thousand Oaks, CA: Sage Publications. 179194.

Bosk, C. (2005). Continuity and change in the study of medical error, the culture of safety on the shop floor. Retrieved from https://www.sss.ias.edu/files/papers/paper20.pdf

Bourgeault, L., Mulvale, G. (2006). Collaborative health care teams in Canada and the USA: confronting the structural embeddedness of medical dominance. Health Sociology Review, 15, 5, 481-495. 
Bourdieu, P. (1998). Practical reason. Stanford, US. Stanford University Press.

Boyce, R. (2006). Emerging from the shadow of medicine: allied health as a 'profession community' subculture. Health Sociology Review, 15, 5, 520-534.

Braithwaite, J., Westbrook, M., Travaglia, J., Huges, C. (2010). Cultural and associated enablers of, and barriers to, adverse incident reporting. Quality and Safety in Health Care,19,3, 229-223.

Brennan, T. Leape, L., Laird, N., Herbert, L., Localio, A., Lawthers, A., Newhouse, J., Weiler, P., Hiatt, H. (1991). Incidence of adverse events and negligence in hospitalised patients. New England Journal of Medicine, 324, 370-376.

Brinkman, A. (2007). Overcoming nursing short-sightedness. Kai Tiaki: Nursing New Zealand, 13, 7, 25

Broom, A., Willis, E. (2007). Competing paradigms and health research. In Saks M., Allsop, J., Researching health. Qualitative, quantitative and mixed methods. 16-31. London, UK: Sage Publications.

Brown, P., MacArthur, C., Newby, L., Lay-yee, R., Davis, P., Briant, R. (2002). Cost of medical injury in New Zealand: a retrospective cohort study. Journal of Health Services Research and Policy 7, 12 29-34

Bryman, A., (2008). Social research methods $3^{\text {rd }}$ Edition.UK: Oxford University Press.

Carayon, P. (2012). Human factors and ergonomics in healthcare and patient safety. Florida, US: CRC Press.

Chang, A., Schyve, P., Croteau, R., O'Leary, D., Loeb, J. (2005). The JCAHO patient safety event taxonomy: a standardized terminology and classification schema for near misses and adverse events. International Journal for Quality in Health Care 17, 2, 95-105.

Chen, H., Hung- Hui, L. (2010). Measuring Patient Safety Culture in Taiwan using the hospitals survey on patient safety culture (HSOPSC) BioMed Central Health Services Research 10, 152. doi: 10.1186/1472-6963-10-152 
Christiaans-Dingelhoff, I., Smitts, M., Zwaan, L., Lubberding, S., Van der Wal, G., Wagner, C. (2011). To what extent are adverse events found in patient records reported by patients and healthcare professionals via complaints, claims and incident reports. BioMed Central Health Services Research. doi:10.1186/1472-6963-11-49

Coal face. (2016) In Cambridge online dictionary. Retrieved from http://dictionary.cambridge.org/dictionary/english/at-the-coalface

Cohen, M. (1999). Medication errors. Washington, US. American Pharmaceutical Association.

Coleman, J. (2014). Speech to NZ Emergency Departments Conference. Retrieved from https://www.beehive.govt.nz/speech/speech-nz-emergency-departmentsconference-taupo

Colla, J., Bracken, A., Kinney, L., Weeks, W. (2005). Measuring Safety Climate: a review of surveys. Quality Safety Health Care, 14, 364-366.

Collins, D. (2010). Safety culture policy statement. Retrieved from http://pbadupws.nrc.gov/docs/ML1006/ML100621266.pdf

Cook, R., Rasmussen, J. (2005). 'Going solid': A model of system dynamics and consequences for patient safety. Quality Safety Healthcare, 14, 130-134.

Cooper, J., Newbower R., Long C., McPeek, B. (1978). Preventable anaesthesia mishaps: a study of human factors. Anaesthesiology, 49, 6, 399-406

Corke, C., De Leeuw, E., Kai Lo, S., George, C. (2009). Predicting future intensive care demand in Australia. Critical care and resuscitation: Journal of the Australasian Academy of Critical Care Medicine, 11, 4, 257-60.

Coster, G. (2013). The role of leadership in enhancing quality. Wellington, New Zealand: Ministry of Health.

Council of the European Union. (2009). Council recommendations on patient safety and the prevention of hospital acquired infections. Retrieved from http://ec.e uropa.eu/health/patient safety/docs/council_2009_en.pdf 
Cresswell, J. (2014). Research design: Qualitative, quantitative and mixed method approaches. Forth Edition. Thousand Oaks, California, United States: SAGE publications.

Cresswell, J. (2009). Research design: Qualitative, quantitative and mixed method approaches. Third Edition. Thousand Oaks, California, United States: SAGE publications.

Davis, P., Lay-Yee, R., Briant, R., (2002). Adverse events in New Zealand public hospitals: Occurrence and impact New Zealand Medical Journal, 1167, 271.

Davis, R., Jacklin R., Sevdalis, N., Vincent, C. (2007). Patient involvement in patient safety: what factors influence patient participation and engagement? Health Expect. 10, 3, 259-67.

Deal, T., Kennedy, A. (1982). Corporate cultures: The rites and rituals of corporate life. Reading, US: Addison-Wesley Publishing.

Dekker, S. (2015). Safety differently: Human factors for a new era 2Ed. Florida, US. CRC Press.

Dekker, S. (2013). Second Victim: Error, guilt, trauma, and resilience. Florida, US. CRC Press

Dekker, S. (2014). The bureaucratization of safety. Safety Science, 70, 348-57.

Department of Health. Berwick, D. chair. (2013). A promise to learn, a commitment to act: Improving the safety of patients in England. National Patient Advisory Group. London, UK: Crown Copyright.

Department of Health. Francis, R. chair. (2013). Report of the Mid Staffordshire NHS Foundation Trust Public Enquiry. London, UK: Crown Copyright.

Department of Health. Kennedy, I. chair. (2001). The report of the public inquiry into children's heart surgery at the Bristol Royal Infirmary 1984-1995: learning from Bristol. London, UK: Crown copyright. 
Department of Health. (2007). Learning from tragedy, keeping patients safe: Overview of the Government's action programme in response to the recommendations of the Shipman Inquiry. London, UK: Crown copyright.

DesRoches, C., Rao, S., Fromson, J., Bimbaum, R., Lezzoni, L., Vogeli, C., Campbell, E. (2010). Physicians' perceptions, preparedness for reporting, and experiences related to impaired and incompetent Colleagues. Journal of the American Medical Association, 304,187-93.

Diamandis, E. (2015). The hundred-person wellness project and Google's baseline study: medical revolution or unnecessary and potentially harmful over-testing? BMC Medicine 13:5 doi: 10.1186/s12916-014-0239-6

Dickson-Swift, V James E., Liamputton. P., (2008). Undertaking sensitive research in the health and social sciences: Managing boundaries, emotions and risks. Cambridge University Press New York.

Dixon-Woods, M., Myles, L., Tarrant, C., Bion, J. (2013). Explaining Matching Michigan: an ethnographic study of a patient safety program. Implementation Science, 8, 70, 1-13.

Dixon-Woods, M., Suokas, A., Tarrent, C. (2009). An ethnographic study of classifying and accounting for risk at the sharp end of medical wards. Social Science and Medicine, 69, 362-369.

Doody, O., Slevin, E., Taggert, L. (2013). Preparing for and conducting focus groups in nursing research: part 2 British Journal of Nursing, 22, 3

Doyle, C., Lennox L., Bell, D. (2013). A systematic review of evidence on the links between patient experience and clinical safety and effectiveness. British Medical Journal Open Access. doi:10.1136/bmjopen-2012-001570

Drach-Zahavy, A., Somech, A. (2011). in Rowley E and Waring J Eds. A sociocultural perspective on patient safety. Farnham. UK. Ashgate publishing.

Edwards, J., Davey, J., Armstrong, K. (2013). Returning to the roots of culture: a review and re-conceptualisation of safety culture. Safety Science, 55, 70-80. 
El-Jardali, F., Jaafar, M., Dimassi, H., Jamal, D., Hamdan, R. (2010). The current state of patient safety culture in Lebanese hospitals: a study at baseline. International Journal for Quality, 22, 5, 386-95.

El-Jardali, F., Sheikh, F., Garcia, N., Jamal, D., Abdo, A. (2014). Patient safety culture in a large teaching hospital in Riyadh: Baseline assessment, comparative analysis and opportunities for improvement. BMC Health Services Research, 10, 152. Retrieved from http://www.biomedcentral.com/content/pdf/1472-6963-14122.pdf

Elpern, E., Covert, B., Kleinpell, R. (2005). Moral distress of staff nurses in a medical intensive care unit. American Journal of Critical Care, 14, 523-530.

Else, A. (2010). Getting it Right: The Cartwright Report Twenty Years On. Retrieved from http://www.wsanz.org.nz/journal/docs/WSJNZ242Else2-7.pdf

Emanuel, L., Berwick, D., Conway, J. Combes, J., Hatlie, M., Leape, L., Reason, J., Schyve, P., Vincent, C., Walton, M. (2008). What Exactly Is Patient Safety? In Henrikson, K. Battles, JB., Keyes MA (Eds.) Advances in patient safety: New directions and alternative approaches (Vol 1: Assessment) Retrieved from https://www.ncbi.nlm.nih.gov/books/NBK43629/

Evans, S., Berry, J., Smith, B., Esterman, A., Selim, P., O'Shaughnessy, J., DeWit, M. (2006). Attitudes and barriers to incident reporting: A collaborative hospital study. Quality Safety Health Care, 15, 1, 39-43. doi:10.1136/qshc.2004.012559

Evershed, N. (2015). Aircraft accident rates at historic low despite high-profile plane crashes. The Guardian, UK. Retrieved from https://www.theguardian.com/world/datablog/2014/dec/29/aircraft-accident-rates-athistoric-low-despite-high-profile-plane-crashes

Ezzy, D. (2002). Qualitative analysis: Practice and innovation. London, Routledge. Press

Feng, X., Bobay, K., Weiss, M. (2008). Patient safety culture in nursing: A dimensional concept analysis. Journal of Advanced Nursing, 63, 3 310-9. doi: 10.1111/j.1365-2648.2008.04728.x. 
Flannagan, J., (1954). The Critical Incident Technique. Psychological Bulletin, 51(4). Retrieved https://www.apa.org/pubs/databases/psycinfo/cit-article.pdf

Flin, R., Fletcher, G., McGeorge, P., Sutherland, A., Patey, R. (2003). Anaesthetists' attitudes to teamwork and safety. Anaesthesia, 58, 233-42.

Freeman, P., Parke, T. (2010). The Future of Acute Care in NZ. The New Zealand Medical Journal. 123 1316. Retrieved from http://www.nzma.org.nz/journal/123$1316 / 4167 /$

Gabler, N., Ratcliffe, S., Wagner, J., Asch, D., Rubenfeld, G., Angus, D., Halpern, S. (2013). Mortality among patients admitted to strained intensive care units. American Journal Respiratory Critical Care Medicine, 188, 800-806.

Gadamer, H. (2004). Truth and method: 2nd Edition (revised) London, UK: Continuum Publishing. Retrieved https://mvlindsey.files.wordpress.com/2015/08/truth-and-method-gadamer-2004.pdf Gauld, R., Hornsburgh, S. (2012). Clinical governance assurance project: Analysis of three quality and safety questions in a national survey of New Zealand health professionals. Centre for health systems. University of Otago. Retrieved from http://www.otago.ac.nz/healthsystems/otago065238.pdf

Gawande, A. (2014). Being mortal: Medicine and what matters in the end. $6^{\text {th }}$ Ed Chicago, US: Metropolitan Books.

Gawande, A. (2010). The checklist manifesto: How to get things right. New York, US: Picador.

GE Healthcare. (2011). Patient safety challenges in nursing identified in global survey. Retrieved from http://newsroom.gehealthcare.com/patient-safety-challengesin-nursing-identified-in-global-survey/

Geertz, C. (1973). The Interpretation of Cultures: Selected essays. New York, US: Basic Books.

Glasser B., Moreno, J., (1989). The qualitative- quantitative distinction in the social sciences. Boston, US: Kluwer Academic 
Grabowski, M., Roberts, K., (1997). Risk mitigation in large-scale systems: Lessons from High Reliability Organizations California Management Review, 39,4 152.

Grbich, C., (2007). Qualitative Data Analysis: An Introduction. SAGE Publications Griffin, F., Resar, R. (2009). IHI Global Trigger Tool for Measuring Adverse Events: Second Edition. IHI Innovation Series white paper. Cambridge, MA: Institute for Healthcare Improvement

Guldenmund, F. (2007). The use of questionnaires in safety culture research: An evaluation. Safety Science, 45, 6, 723-743.

Haerkens, M., Kox, M., Lemson, J., Houterman, S., Van der Hoeven, J., Pickkers, P. (2015). Crew resource management in the intensive care unit: A prospective 3-year cohort study. Acta Anasthesiologica Scandanavia 59, $101319-29$

Hale, A., Borys, D. (2013b). Working to rule, or working safely? Part1: A state of the art review. Safety Science, 55, 207-221.

Hale, A., Borys, D., (2013a). Working to rule or working safely? Part 2: The management of safety rules and procedures. Safety Science, 55, 222-231.

Hall, E. T. (1976). Beyond culture. Garden City, N.Y: Anchor Press

Handel, D., Hilton, J., Ward, M., Rabin, E., Zwemer, F., Pines, J. (2010). Emergency department throughput, crowding, and financial outcomes for hospitals. Academic Emergency Medicine, 17, 840-847.

Hardy, L. (2013). How do district health boards respond to and use the serious and sentinel event report? A thesis submitted for the degree of Masters of Public Health. (unpublished thesis) University of Otago, Dunedin. NZ

Harm. (2009). In The free dictionary.com. Retrieved from http://medicaldictionary.thefreedictionary.com/harm

Hartmann, CW., Rosen, AK., Meterko, M., Shokeen, P., Zhao, S., Singer, S., Falwell, A., Gaba, DM. (2008). An overview of patient safety climate in the VA. Health Services Research, 43, 1264-1484 
Haukelid, K. (2008). Theories of (safety) culture revisited: An anthropological approach. Safety Science, 46,3, 413-427

Health and Disability Commission. (2016b). Decisions and case notes. Retrieved from http://www.hdc.org.nz/decisions--case-notes

Health and Disability Commission. (2016a). The role of the health and disability commissioner and the code of health and disability services consumers' rights Retrieved from http://www.hdc.org.nz/education/presentations/the-role-of-the-healthand-disability-commissioner-and-the-code-of-health-and-disability-servicesconsumers'-rights

Health and Disability Commission. (2013). Health and disability commission annual report. Health and Disability Commissioner. Auckland. New Zealand Health and Safety Executive. (2005). A review of safety culture and safety climate literature for the development of the safety culture inspection toolkit. London, UK: Crown Copyright.

Health and Safety Executive. (1993). ACSNI Study group on human factors: Third report - organising for safety. London, UK: Crown Copyright.

Health Quality and Safety Commission. (2015c). Adverse events reports. Retrieved http://www.hqsc.govt.nz/our-programmes/adverse-events/serious-adverse-eventsreports/

Health Quality and Safety Commission. (2016c). Co designing healthcare. Retrieved from http://www.hqsc.govt.nz/our-programmes/partners-in-care/work-streams/codesign-partners-in-care/

Health Quality and Safety Commission. (2016b). Deteriorating adult patient evidence summary. Wellington, NZ: HQSC.

Health Quality \& Safety Commission. (2015d). Learning from adverse events: Adverse events reported to the Health Quality \& Safety Commission 1 July 2014 to 30 June 2015. Wellington, NZ: HQSC. 
Health Quality and Safety Commission. (2015d). Learning from adverse events focus of report http://www.hqsc.govt.nz/news-and-events/media/2385

Health Quality and Safety Commission. (2015b). Our role. Retrieved from http://www.hqsc.govt.nz/about-the-commission/our-role/

Health Quality \& Safety Commission. (2010). Making our hospitals safer: Serious and sentinel events 2009/10. Wellington, NZ: HQSC. Retrieved from http://www.hqsc.govt.nz/our-programmes/adverse-events/publications-andresources/publication/155/

Health Quality and Safety Commission. (2016a). National patient survey: Results for patients treated in February 2016. Retrieved from http://www.hqsc.govt.nz/ourprogrammes/health-quality-evaluation/publications-andresources/publication/2513/\#[NATIONAL]

Health Quality and Safety Commission. (2015a). Safer Surgery: Survey shows surgical teams working well together. Retrieved from http://www.hqsc.govt.nz/ourprogrammes/safe-surgery-nz/news-and-events/media/2407/

Heard, G., Sanderson, P., Thomas, R. (2012). Barriers to adverse event and error reporting in anaesthesia. Anaesthesia and Analgesia 114, 604-614.

Hellings, J., Schrooten, W., Klazinga, N., Vleugels, A. (2007). Challenging patient safety culture: survey results, International Journal of Health Care Quality Assurance, 20,7620 - 632 .

Helmreich, R. (2000). On error management: lessons from aviation. British Medical Journal, 320, 781-784.

Helmreich, L., Schaefer, G. (1994). Team performance in the operating room. In Bogner, M., Human Error in Medicine. New Jersey, US: Erlbaum Associates.

Hemman, E., (2002). Creating healthcare cultures of patient safety. The journal of Nursing Administration 32 (7-8) 419-27Hofstede, G., Hofstede, GJ., Minkov, M. (2010). Cultures and Organizations: Software of the Mind, Third Edition. New York, US: McGraw-Hill Education. 
Hollnagel, E. Pariès, J. Woods, D., Wreathall, J. (Eds.) (2010). Resilience Engineering Perspectives: Resilience Engineering in Practice. Farnham, UK: Ashgate. Retrieved from http://erikhollnagel.com/books/resilience-engineering-inpractice.html

Hollnagel, E., (2014). From Safety, I to Safety II: The past and future of safety management. Surrey, UK: Ashgate publishing

Hollnagel, E., Wears, R., Braithwaite, J. (2015). From Safety-I to Safety- II: A white paper. The Resilient Healthcare Net: Published simultaneously by the University of Southern Denmark, University of Florida and Macquarie University, Australia. Retrieved from http://resilienthealthcare.net/onewebmedia/WhitePaperFinal.pdf Holloway, I., Wheeler, S. (2010). Qualitative research in nursing and healthcare $3^{\text {rd }}$ edition. London, UK: Blackwell Science.

Hoot, N., Aronsky, D. (2008). Systematic review of ED crowding: Causes, effects and solutions. Annuals of Emergency Medicine 49, 747-755.

Huang, D., Clermount, G., Kong, L., Weissfeld, L., Sexton, J., Rowan, K., Angus, D. (2010) Intensive care unit safety culture and outcomes: A US multicentre study. International Journal for Quality in Health Care, 22,3 151-161.

Hughes, E. (1951). Mistakes at work. Canadian Journal of Economics and Political Science, 17, 320-7.

Injury. (2016). In The freedictionary.com. Retrieved from http://medicaldictionary.thefreedictionary.com/injury

Institute of Healthcare Improvement. (2016). Develop a culture of safety. Retrieved from http://www.ihi.org/resources/Pages/Changes/DevelopaCultureofSafety.aspx Institute of Healthcare Improvement. (2006). Leadership guide to patient safety. Cambridge, US: IHI.

Institute of Medicine. (2001). Crossing the quality chasm: A new health system for the 21st century. Washington, DC: National Academy Press. 
Institute of Medicine. (2000). Kohn, L., Corrigan, J., Donaldson, M. (Eds.) To err is human: Building a safer health system. Washington, D.C: National Academy Press. Institute of Nuclear Power Operators. (2013). Traits of a healthy nuclear safety culture addendum I: Behaviours and actions that support a healthy nuclear safety culture, by organizational level. INPO. Crown Copyright. Retrieved from nrc.gov. International Nuclear Safety Advisory Group. (1991). Safety culture: A report by the international nuclear safety advisory group. Vienna, Austria: International Atomic Energy Agency.

Jiang, N., Pacheco G. (2014). Demand in New Zealand hospitals: Expect the unexpected? Retrieved from https://www.aut.ac.nz/_data/assets/pdf_file/0005/486338/Economics-WP-201404.pdf

Johnson, M., (2013). Evaluating culture: Well-being, institutions and circumstance. London, UK: Springer.

Kallberg, A., Gorannsson, Florin, J., Ostergren, J., Brixey, J., Ehrenberg. (2015). Contributing factors to errors in Swedish emergency departments. International Emergency Nursing, 23, 151-161

Kanter, R. (1993). Men and women of the corporation (2nd ed.). New York, NY: Basic Books.

Keene, L., Bagshaw, P., Gary Nichols, M., Rosenberg, B., Frampton, C., Powell, I. (2016). Funding New Zealand's public healthcare system: Time for an honest appraisal and public debate. New Zealand Medical Journal, 129, 1435, 10-20 Keogh, B. (2013). Review into the quality of care and treatment provided by 14 hospital trusts in England: Overview report. London, UK. Crown Copyright.

Kingston, M., Evans, S., Smith, B., Berry, J. (2004). Attitudes of doctors and nurses towards incident reporting: A qualitative analysis. Medical Journal of Australia 181,1 36-39 
Kitch, B. (2008). Safety culture. In Ashish J (Ed). World Health Organization alliance for patient safety. Summary of the evidence on patient safety: Implications for research. World Health Organization, Geneva, 54-56

Krueger, R. (1994). Focus Groups: A practical guide for applied research. Michigan, US: Sage Publications.

Krueger, R., Casey, M. (2015). Focus groups: A practical guide for applied research. Thousand Oaks, US: SAGE.

Kvale, S., Brinkman, S. (2009). Interviews: Learning the craft of qualitative research interviewing. Thousand Oaks, US: SAGE.

Lankshear, A.J., Sheldon, T.A., Maynard, A., (2005). Nurse staffing and healthcare outcomes. A systematic review of the international research evidence. Advances in Nursing Science, 28, 2163-174.

Lawrenson, R., Waetford, J., Gibbons, V., Kirk, P. Haggar, S., Reddy, R. (2012). Palliative care patients use of emergency departments. NZ Medical Journal,126 (1327). Retrieved from https://www.nzma.org.nz/journal/read-the-journal/allissues/2010-2019/2013/vol-126-no-1372/article-lawrenson.

Leape, L. (2009). Errors in medicine. Clinical Chimica Acta, 404, $12-5$

Leape, L. (2015). Patient safety in the era of healthcare reform. Clinical Orthopaedic Related Research. May; 473(5): 1568-1573. doi: 10.1007/s11999-014-3598-6Leape, L. (2002b). Reporting of adverse events. New England Journal of Medicine, 347, 1633-1638.

Leape, L. (2002). Striving for perfection. Seven steps to patient safety. Clinical Chemistry 48, 11,1871-2

Leape, L., Berwick, D. (2005). Five years after 'To Err is Human': What have we learned? Journal of the American Medical Association, 293,19, 2384-90

Leape, L., Kabcenell, A., Berwick, D. (1998). Reducing adverse drug events and medical errors. Boston, US: Institute for Healthcare Improvement. 
Leape, L., Lawthers, A., Brennan, T., Johnson, W. (1993), Preventing medical injury. Quality Review Bulletin, 19, 144-9

Leistikow, I., Mulder, S., Vesseur, J., Robben, P. (2016). Learning from incidents in healthcare: the journey, not the arrival, matters. BMJ Quality Safety. Retrieved from doi:10.1136/bmjqs-2015-004853

Lincoln, Y., Guba, E. (1985). Naturalistic Inquiry. Newbury Park, CA: Sage Publications.

Little, D. (2008). What is hermeneutic explanation? Retrieved from http://www.personal.umd.umich.edu/ delittle/Encyclopedia\%20entries/hermeneutic\% 20explanation.htm

Ludwick, R., Silva, M.C., (2000). Ethics: Nursing Around the World: Cultural Values and Ethical Conflicts Online Journal of Issues in Nursing, 5, 3, Retrieved from http://www.nursingworld.org/MainMenuCategories/ANAMarketplace/ANAPeriodicals/ OJIN/TableofContents/Volume52000/No3Sept00/CulturalValuesandEthicalConflicts. html

Marginalisation (2016) In: The business dictionary.com Retrieved from: http://www.businessdictionary.com/definition/marginalization.html\#ixzz3R7JjshiQ Makary, M., Mukherjee, A., Sexton, B., Syin, D., Goodrich, E., Hartmann, E., Rowen, L., Behrens, Drew, C., Marohn, M., Pronovost, P. (2007). Operating Room Briefings and Wrong-Site Surgery. Journal of the American College of Surgeons, 204,2, 236243.

Makary, M., Sexton, JB., Freischlag, J., Holzmueller, C., Millman, E., Rowen, L., Pronovost, P. (2006). Operating room teamwork among physicians and nurses: Teamwork in the eye of the beholder. Journal of American College of Surgeons. 202, $5,746-52$.

Makary, M., Sexton, JB., Freischlag, JA., Millman, EA., Pryor, D., Holzmueller, C., Pronovost, PJ. (2006). Patient safety in surgery. Annuals of Surgery, 243, 5, 628-32. 
Mamouni Liminos, E., Mazzarol, T., Ghadouani, A., Schizzi, S. (2014). The resilience architecture framework: Four organizational archetypes. European Management Journal, 32,1, 104-116.

Martin, G., Mason, D., Lovelock, K., Cumming, J., Hider, P. (2015). Health professional's perceptions of quality survey: A report for the HQSC. Retrieved from https://www.hqsc.govt.nz/assets/General-PR-files-images/Perceptions-of-qualitysurvey-Oct-2015.pdf

Martin, J. (2001). Organisational culture: Mapping the terrain. SAGE Publications Mauro, F. (2016). Patient safety is not a luxury. The Lancet, 3871133 McCannon, J., Berwick, D. (2011). A new frontier in patient safety. Journal of the American Medical Association 305 (21) 2221-2.

McDermid, F., Peters, K., Jackson, D., Daly, J. (2014). Conducting qualitative research in the context of pre-existing peer and collegial relationships. Nurse Researcher. 21,5, 28-33.

McDonald, R., Waring, J., Harrison, S., Walshe, K., Boaden, R. (2005). Rules and guidelines in clinical practices: A qualitative study in operating theatres of doctors and nurses' views. Quality Safety Healthcare 14, 290-294.

Mcloughlin, V., Millar, J., Mattke, S., Franca, M., Jonsson, P., Bates, D. (2006). Selecting indicators for patient safety at the health care system level in OECD countries. International Journal for Quality in Health Care, 14. 20.

Medical Council of NZ. (2010. Disclosure of harm following an adverse event. Retrieved from https://www.mcnz.org.nz/assets/News-andPublications/Statements/Disclosure-of-harm.pdf Merry, A. (2015). Learning from adverse events focus of report. Retrieved from http://www.hqsc.govt.nz/news-and-events/media/2385

Miles, M., Huberman, A. (1984). Qualitative data analysis. London, UK: Sage. 
Mills, J., Bonner, A., Francis, K. (2006). Adopting a constructivist approach to grounded theory: Implications for research design International Journal of Nursing Practice, 12, 1, 8-13

Ministry for Culture and Heritage. (2016). The Treaty in brief, Retrieved from http://www.nzhistory.net.nz/politics/treaty/the-treaty-in-brief.

Ministry of Health New Zealand. (2001). Adverse events in New Zealand public hospitals: Principle findings from a national survey. Retrieved from https://www.health.govt.nz/system/files/documents/publications/adverseevents.pdf Ministry of Health. (2001). A framework for credentialing. Retrieved from https://www.health.govt.nz/system/files/documents/publications/clinical.pdf Ministry of Health. (2014). A quality framework and suite of quality measures for the emergency department phase of acute patient care in NZ. Retrieved from http://www.health.govt.nz/publication/quality-framework-and-suite-quality-measuresemergency-department-phase-acute-patient-care-new)

Ministry of Health. (2016b). Emergency departments Retrieved from http://www.health.govt.nz/our-work/hospitals-and-specialist-care/emergencydepartments

Ministry of Health New Zealand. (2011). Emergency department triage. Retrieved from http://www.health.govt.nz/our-work/hospitals-and-specialist-care/emergencydepartments/emergency-department-triage

Ministry of Health. (2016a). Health strategy update. Retrieved from http://www.health.govt.nz/system/files/doc uments/publications/update-new-zealandhealth-strategy-consultation-draft-oct15_0.pdf

Ministry of Health. (2012). Hospital events 2008/09 and 2009/10. Retrieved from http://www.health.govt.nz/publication/hospital-events-2008-09-and-2009-10 Ministry of Health. (1998). Hospital services plan. Setting the scene. Retrieved from http://www.moh.govt.nz/moh.nsf/Files/hospservb/\$file/hospservb.pdf 
Ministry of Health New Zealand. (2005). Intensive care services in New Zealand: A report to the deputy director-general, clinical services from the intensive care clinical advisory group. Ministry of Health New Zealand, Wellington.

Mitka, M. (2008). Emergency departments see high rates of adverse events from antibiotic use. Journal of the American Medical Association, 300,13, 1505-1506. doi:10.1001/jama.300.13.1505.

Morgan, D. (1997). Focus groups as qualitative research. Michigan. Sage Publications.

Nabhan, M., Elraiyah, T., Brown, D., Dilling, J., LeBlanc, A., Montori, V., Morgenthaler, T., Naessens, J., Prokop, L., Roger, V., Swensen, S., Thompson, R., Murad $\mathrm{H}$ (2012) What is preventable harm in healthcare? A systematic review of definitions BMC Health Services Research. Retrieved from http://bmchealthservres.biomedcentral.com/articles/10.1186/1472-6963-12-128 National Confidential Enquiry into Patient Outcomes and Death. (2012). Time to intervene: $A$ review of patients who underwent $C P R$ as a result of an in hospital cardiorespiratory arrest. NCEPOD. UK

National Ethics Advisory Committee. (2013). The ethical principle of 'do no harm' and industrial action. Wellington: Ministry of Health.

National Patient Safety Agency. (2008). A risk matrix for risk managers. London. NHS NPSA.

National Patient Safety Agency. (2004). Seven steps to patient safety. NHS. London. United Kingdom.

National Patient Safety Foundation. (2015). Free from harm: Accelerating patient safety improvement fifteen years after To Err is Human. National Patient Safety Foundation. US Boston.

Newbower, RS., Long, CD., McPeek, B. (1978). Preventable anaesthesia mishaps: a study of human factors. Anaesthesiology, 49, 6, 399-406.

New Zealand Medical Council. (2006) Good Medical Practice. Retrieved from http://www.mcnz.org.nz/assets/News-and-Publications/good-medical-practice.pdf 
Nursing Council of NZ. (2012). Code of Conduct. Retrieved from http://www.nursingcouncil.org.nz/Nurses/Code-of-Conduct

Nugus, P., Braithwaite, J. (2010). The dynamic interaction of quality and efficiency in the ED: Squaring the circle? Social science and medicine, 70, 511-517

Occupational culture (2016) In Psychology Dictionary Online. Retrieved fromhttp://psychologydictionary.org/occupational-culture/

O'Malley, S., Gerland, M., Pham, H., Berenson, A. (2005). Rising pressure: hospital emergency departments as barometers of the health care system. Issue Brief Central Studies Health System Change 101, 1-4.

Onwuegbuzie, A., Dickinson W, Leech, N., Zoran., A (2009). A qualitative framework for collecting and analysing date in focus group research. International Journal of Qualitative Methods 8 (3) Retrieved from http://research.apc.org/images/2/2f/A_Qualitative_Framework_for_Collecting_and_A nalyzing_Data_in_Focus_Group_Research.pdf

Oster, C., Strong, J., Zorn, C. (2013). Analysing aviation safety: Problems, challenges, opportunities. Research in Transportation Economics, 43 148-164

O'Reilly, C., Chapman, J. (1996). Culture as social control: Corporations, cults and commitment. Research in Organisational Behaviour,18, 157-200.

Papadimos, T., Maldonado, Y., Tripathi, R., Kothari, D., Rosenburg, A. (2011). An overview of end-of-life issues in the intensive care unit. International Journal of Critical IIIness and Injury science, 1, 2, 138-146.

Parry, G., Cline, A., Goldmann, D. (2012). Deciphering harm measurement. Journal of the American Medical Association, 23, 307 (20), 2155-2156.

Patton, M. Q. (2015). Qualitative research \& evaluation methods: Integrating theory and practice: the definitive text of qualitative inquiry frameworks and options (Fourth edition.). Thousand Oaks, California: SAGE Publications. 
Perrow, C., (1984). Normal Accidents: Living with High-Risk Technologies. Princeton New Jersey. Princeton University Press.

Perry, S., Wears, R., Fairbanks., R (2012). Chapter 41: Human Factors and ergonomics in the emergency department. In Carayon, P. (Ed.) Human factors and ergonomics in healthcare and patient safety. CRC Press. Florida US

Person, J., Spiva L., Hart, P. (2012). The culture of an emergency department: An ethnographic study. International Emergency Nursing 21 222-227

Plummer-D'Amato, P (2008). Focus group methodology Part 2: Considerations for analysis. International Journal of Therapy and Rehabilitation. 15, 3

Pope, C., Ziebland, S., Mays, N. (2000). Analysing qualitative data. British Medical Journal. 8; 320(7227): 114-116.

Pronovost, P., Colantuoni, E., (2009). Measuring preventable harm: Helping science keep pace with policy. Journal of the American Medical Association. 301,12, 12731275.

Pronovost, P., Weast, B., Holzmueller, C., Rosenstein, B., Kidwell, B., Haller, K., Feroli, E., Sexton, J., Rubin, H. (2003). Evaluation of the culture of safety: A survey of clinicians and managers in an academic medical centre. Quality Safety Health Care, 12, 405-410.

Pronovost, P, Sexton, B. (2005). Assessing safety culture: guidelines and recommendations. Quality Safety Health Care, 14, 231-233.

Rafferty, AM., Clarke, S., Coles, J., Ball, J., James, P., McKee, M. Aiken, L. (2007). Outcomes of variation in hospital nurse staffing in English hospitals: cross-sectional analysis of survey data and discharge records. International Journal of Nursing Studies 44, 2, 175-182.

Rathert, C., Williams, E., Lawrence, E., Halbesleben, J. (2012). Emotional exhaustion and workarounds in acute care: Cross sectional tests of a theoretical framework. International Journal of Nursing Studies, 49, 8, 969-977.

Reason, J., Hobbs, A. (2003). Managing maintenance error. US, CRC Press. 
Reason, J. (1991). Human error. Cambridge Press: United Kingdom.

Reason, J. (1997). Managing the risks of organisational accidents. Ashgate publishing.

Reason, J. (1990). The Contribution of latent human failures to the breakdown of complex systems. Philosophical Transactions of the Royal Society of London. Series B, Biological Sciences 327, 1241, 475-484.

Reason, J. (2008). The human contribution: Unsafe acts, accidents and heroic recoveries. CRC Press, Florida, US.

Reason, J. (2000). Human error: Models and management. Western Journal of Medicine, 172, 6, 393-6.

Redmond, R., Curtis, E. (2009). Focus Groups. Principles and Process. Nurse Researcher, 16, 3, 57-69.

Richter, A., Koch, C. (2004). Integration, differentiation and ambiguity in safety cultures. Safety Science, 42, 8, 703-722.

Ring. (2010). Case 34: A 65-year-old woman with an incorrect operation on the left hand. New England Journal of Medicine, 363, 20 1950-1957.

Ring, L., Fairchild, R. (2013). Leadership and patient safety: A review of the literature. Journal of Nursing Regulation, 4, 152-55.

Robb, G., Seddon, M. (2010). Measuring the safety culture in a hospital setting. A concept whose time has come. New Zealand Medical Journal. Retrieved from http://www.nzma.org.nz/journal/123-1314/4112

Rowley, E., Waring, J Eds. (2011). A sociocultural perspective on patient safety. Ashgate publishing. Farnham. UK.

Royal College of Nursing. (2008). Defending dignity: Challenges and opportunities for nursing. London: Royal College of Nursing. Retrieved from www.rcn.org.uk/_data/assets/pdf_file/0011/166655/003257.pdf.

Rubin, H. Rubin, I. (2012). Qualitative interviewing: The art of hearing data, third edition. Thousand Oaks, California, United States: SAGE publications 
Runciman, W., Hibbert, P., Thomson, R., Van Der Scheaff, T., Sherman, H., Lewalle P. (2009). Towards an International Classification for Patient Safety: Key concepts and terms. International Journal for Quality in Health care 21, 1, 18-26.

Saldana, J. (2009). The Coding Manual for Qualitative Researchers. London. SAGE publications.

Samner, C., Lykens, K., Singh, K., Mains, D., Lackan, N. (2010). What is patient safety culture? A review of the literature. Journal of Nursing Scholarship. 42, 2,15665.

Sari, A., Sheldon, T., Cracknell, A., Turnbull, A. (2007). Sensitivity of a routine system for reporting patient safety incidents in an NHS hospital: retrospective patient case not review. British Medical Journal, 334, 7584, 79.

Schein, E. (2004). Organizational culture and leadership. San Francisco: JosseyBass Publishers.

Scott, T., Mannion, R., Davies, H., Marshall, M. (2003). The quantitative measurement of organizational culture in health care: A review of the available instruments. Health Service Research, 38 3 923-945.

Seddon, M. (2003). Aiming for a quality health service: quality improvement in the New Zealand health sector. In: Gauld R (Ed.) Continuity and chaos, healthcare re management and delivery in New Zealand. Dunedin. New Zealand University of Otago Press

Seddon, M. (2006). Effective practice and quality improvement in healthcare in New Zealand. Part 1: What would a high-quality healthcare system look like? New Zealand Medical Journal, 119,1237, 1-5

Seddon, M. (2012). Reporting hospital performance-a balancing act between accountability and quality improvement. New Zealand Medical Journal Retrieved from http://www.nzma.org.nz/journal/125-1366/5443/

Seddon, M. (2007). Safety of patients in NZ hospitals: A progress report. Retrieved from 
http://www.mhc.govt.nz/media/30166/safety\%20of\%20patients\%20in\%20nz\%20hos pitals, \%20mary\%20seddon.pdf

Seddon, M., Jackson, A., Cameron, C., Young, M., Escott, L., Maharaj, A., Miller, N. (2012). The adverse drug event collaborative: a joint venture to measure medicationrelated patient harm. New Zealand Medical Journal, 25, 126(1368), 9-20.

Sendelbach, S. Funk, M. (2013). Alarm fatigue: a patient safety concern. AACN Advanced Critical Care 24, 4, 378-386

Sexton, JB., Berenholtz, SM., Goeschel, CA., Watson, SR., Holzmueller, CG., Thompson, DA., Hyzy, RC., Marsteller, JA., Schumacher, K., Pronovost, PJ. (2011). Assessing and improving safety climate in a large cohort of intensive care units. Critical Care Medicine, 39, 5, 934-999

Sexton, J., Helmreich, R., Neilands, T., Rowan, K., Vella, K., Boyden J., Roberts, P., Thomas, E .(2006). The safety attitudes questionnaire: Psychometric properties benchmarking data, and emerging research. BMC Health Services Research 6, 443 Sexton, B., Thomas, J., Helmreich, L. (2000). Error, stress and teamwork in medicine and aviation: cross sectional surveys. British Medical Journal (clinical research edition) 320, 745-749.

Shine, K. (2013). Patient safety strategies: A call for physician leadership. Annuals of Internal Medicine, 158 353-354

Silverman, D. (2001). Interpreting qualitative data. 2nd Ed. London, UK: SAGE Singer, SJ., Gaba, DM., Falwell, A., Lin, S., Hayes, J., Baker, L. (2009). Patient safety climate in 92 US hospitals: differences by work area and discipline. Medical Care 47, 23-31.

Singer, S., Gaba, D., Geppert, J., Sinaiko, Howard, S., Park, K. (2003). The culture of safety: results of an organisation-wide survey in 15 Californian hospitals. Quality, Safety, Health Care, 12, 112-118. 
Singla, A., Kitch, B., Weissman, J., Campbell, E. (2006). Assessing patient safety culture: A review and synthesis of the measurement tools. Journal of patient safety, 2, 3,105-15.

Smith, M., Feied, C. (1999). The ED as a complex system. Retrieved from http://www.necsi.edu/projects/yaneer/emergencydeptcx.pdf

Smits, M., Wagner, C., Spreeuwenberg, P., Van der Wal, G., Groenewegen, PP. (2009). Measuring patient safety culture: an assessment of the clustering of responses at unit level and hospital level. Quality and Safety in Health Care, 18, 292-296.

Smits, M., Wagner, C., Spreeuwenberg, P., Timmermans, D., Van der Wal, G. Groenewegen, PP. (2012). The role of patient safety culture in the causation of unintended events in hospitals. Journal of Clinical Nursing, 21, 3392-3401.

Sorra, J., Dyer, N. (2010). Multilevel psychometric properties of the AH Retrieved from: RQ hospital survey on patient safety culture. BMC Health Services Research 10. Retrieved from: http://www.biomedcentral.com/1472-6963/10/199.

Stake, R. (2005). Qualitative case studies. In Denzin, Norman K. \& Lincoln, Y (Eds.) The sage handbook of qualitative research. (Third edition. pgs. 443-466). Thousand Oaks, California: SAGE publications.

Stake, R (1995). The art of care study research. California, United States: SAGE publications.

Stalpers, D., de Brouwer, B., Kaljouw, M., Schuurmans, M. (2015). Associations between characteristics of the nurse work environment and five nurse sensitive patient outcomes in hospitals: a systematic review of the literature. International Journal of Nursing Studies 52 817-835

Standards Australia/New Zealand. (2009). Risk management - principles and guidelines. Sydney/Wellington., Standards Australia/New Zealand

St Pierre, E., Jackson, A. (2014). Qualitative data analysis after coding. Qualitative Inquiry. 20, 6, 715-719. 
Streat, S. In Crippen, D (Ed). (2013). ICU Resource Allocation in the new millennium: Will we say No? Springer Science and Business Media NY65-71

Strauss, A., Corbin, J. (1998). Basics of Qualitative Research, 2nd edition. London: Sage.

Sutcliffe, K. (2011). High Reliability Organisations. Best Practice \& Research Clinical Anaesthesiology, 25,133-144

The Economist. (2015). A Crash course in probability. Retrieved from http://www.economist.com/blogs/gulliver/2015/01/air-safety

Then, K., Rankin, J., Ali, E. (2014). Focus group research: What is it and how can it be used? Canadian Journal of Cardiovascular Nursing, 24, 1, 16-22

The University of Victoria, Wellington. (2016). Research ethics. Retrieved from http://www.victoria.ac.nz/fgr/current-phd/ethics

The University of Victoria. (2016). Human ethics policy. Retrieved from http://www.victoria.ac.nz/documents/policy/research-policy/human-ethics-policy.pdf Traynor, M. (1999). Managerialism and nursing beyond oppression and profession. London, UK: Routledge

Triandis, H. (1989). The self and social behaviour in differing cultural contexts. Psychological Review, 96, 3, 506-520.

Ummenhofer, W., Amsler, F., Sutter, PM., Martina, B., Martin, J., Scheidegger, D. (2001). Team performance in the emergency room: assessment of inter-disciplinary attitudes. Resuscitation, 49, 39-46.

Van der Leeuw, Lombarts, K., Arah, O., Heineman, M. (2012). A systematic review of the effects of residency training on patient outcomes. BMC Medicine 10:65 http://bmcmedicine.biomedcentral.com/articles/10.1186/1741-7015-10-65

Van Maanen, J., Barley, S. (1982). Occupational communities: Culture and control in organisations. MIT Report for the Office of Naval Research. Office of Naval Research. Cambridge. US 
Vaughan, D. (1999). The dark side of organizations: Mistakes, misconduct, and disaster. Annual Review of Sociology, 25 271-305

Vaughn, S., Schumm, J. S., Sinagub, J. (1996). Focus group interviews in education and psychology. Thousand Oaks, Sage.

Victoria University of Wellington. (2016). Research Ethics. Retrieved from http://www.victoria.ac.nz/fgr/current-phd/ethics\#human

Victoria University of Wellington. (2016). Human ethics policy. Retrieved from http://www.victoria.ac.nz/documents/policy/research-policy/human-ethics-policy.pdf

Vincent, C. (2006). Patient safety. Elsevier. London. United Kingdom.

Vincent, C, Amalberti, R. (2016). Safer Healthcare: Strategies for the Real World. Springer. Open access online.

Vincent, C., Coulter, A. (2002). Patient safety: What about the patient? Quality Safety Health, 11, 76-80

Vincent, C. (2010). Reporting and learning systems. In: Vincent C, ed. Patient safety. 2nd ed. Chichester: Wiley Blackwell, 75-95.

Vogus, T., Sutcliff, K. (2007). The safety organising scale: Development and validation of a behavioural measure of safety culture in hospital nursing units. Medical Care 45, 1, 1-9.

Wagner, C., Smits, M., Sorra, J., Huang, C. (2013), Assessing patient safety culture in hospitals across countries. International Journal for Quality in Healthcare 25, 3, 213-221.

Wallis, K., Dovey, S. (2011). Assessing patient safety culture in New Zealand primary care: a pilot study using a modified Manchester Patient Safety Framework in Dunedin general practices. Journal of Primary healthcare, 3,1, 35-39.

Walton, M., Shaw, T., Barnet, S., Ross, J. (2006). Developing a national patient safety education framework for Australia. Quality Safety Healthcare, 15, 437-442.

Waring, J. (2005). Beyond Blame: Cultural barriers to medical incident reporting. Social Science and Medicine, 60, 1927-1935. 
Weick, K., Sutcliffe, K. (2001). Managing the unexpected: Assuring high performance in an age of complexity. San Francisco, US: Jossy Bass.

Weissman, Schneider, Weingart, S., Epstein, A., David-Kasdan, J., Feibelmann, S., Annas, C., Ridley, N., Kirle, L., Gatsonis, C. (2008). Comparing patient reported hospital adverse events with medical record review: Do patients know something that hospitals do not? Annuals of internal Medicine, 149,100-108

Westrum, R. (1993). Cultures with requisite imagination. In Verification and validation of complex systems: Human factors issues: NATO ASI Series. 10, 401-416

Westrum, R., Adamski, A. (1999). Organizational factors associated with safety and mission success in aviation environments. In: Garland, DJ., Wise, JA., Hopkins, VD., (Eds). Aviation human factors. Hillsdale, US: Lawrence Erlbaum

White, C. (2004). Doctors mistrust systems for reporting medical mistakes. British Medical Journal 329:12

World Health Organisation. (2016a). Call for a global movement for patient safety. Retrieved from http://www.who.int/patientsafety/news_events/global-movement/en/ World Health Organisation. (2016b). European Union validation of minimal information model for patient safety incident reporting. Retrieved from http://www.who.int/patientsafety/implementation/taxonomy/eu-mim-validation/en/ World Health Organisation. (2012). Modern health care delivery systems, care coordination and the role of hospitals. A report for the European office. http://www.euro.who.int/_data/assets/pdf_file/0008/158885/BRU-report-Modernhealth-care-delivery-systems.pdf

World Health Organisation. (2014). Ten facts on patient safety. http://www.who.int/features/factfiles/patient_safety/en/index.html (accessed 06/07/2016)

World Health Organisation. (2009). What is patient safety? (accessed 19/04/2016) http://www.who.int/patientsafety/education/curriculum/course1_handout.pdf World Medical Association. (2015). World medical ethics manual $3^{\text {rd }}$ Edition, Belgium: WMA 
Wolf, Z., Hughes, R. (2008). Error reporting and disclosure. In: Hughes RG, editor. Patient safety and quality: An evidence-based handbook for nurses. Rockville (MD): Agency for Healthcare Research and Quality (US). Chapter 35. Available from: http://www.ncbi.nlm.nih.gov/books/NBK2652/

Ybema, S., Yanow, D., Wels, H., Kamsteeg, F. (2009). Organisational ethnography: Studying the complexity of everyday life. London, UK: SAGE publications.

Yin, R. (2009). Case study research design and methods, fourth edition. Thousand Oaks, California, United States: SAGE publications.

Zohar, D., Livne, Y., Tenne-Gazit, O., Admi, H. Donchin, Y. (2007). Healthcare climate: a framework for measuring and improving patient safety. Critical Care Medicine, 35, 1312-1317.

Zuzelo, P. (2007). Exploring the moral distress of registered nurses. Nursing Ethics, 14 (3) 344-358. 


\section{Appendix 1: Interview Guide}

\section{Interview Guide Template:}

\section{Warm up}

General discussion and welcome. Introduction to focus group.

\section{Clarification of terms (10 mins)}

Welcome everyone, and thank you very much to you all for coming today and taking part. My name is Joanna Wailling. I am a student currently studying a Masters in Healthcare Research at Victoria University. My background is in acute care, and my research interest is patient safety.

For focus groups only: This is my colleague (name) who will be observing our discussions today. To recognise your time and contribution to this research, if you would like a certificate of attendance for your portfolio, please see us afterwards and we will complete one.

What is the background and purpose of this research?

I would just like to tell you a bit about the research which is entitled 'How do health care professionals working on acute care environments describe patient safety.'

The study objectives are to:

1. Explore similarities and differences in how doctors and nurses at unit level, and organisational managers experience patient safety.

2. Describe the organisational and clinical factors perceived by healthcare professionals as important in developing a patient safety culture in an acute care hospital.

3. Make recommendations to inform the development of positive patient safety culture for an acute care hospital setting in New Zealand. 


\section{Confidentiality}

We are intending to record the focus groups/interview. Can you please sign the form to give your consent to being recorded? The focus group/interview is recorded to ensure we have an accurate account of the discussion. The recording will be typed up but each person will be given an ID number - in this way no identifiable information will be associated with a named individual. Your responses will be anonymous and the tape will be typed up and destroyed once the analysis is over. Does anyone have any questions about confidentiality that I haven't covered? If not, can you please sign the two copies of the consent form (one for us one for you) and I'll co-sign these before you leave.

- For focus groups only: $X$ will also be taking a few notes and recording the discussion.

If everyone's happy, l'll turn on the recorder. (SWITCH ON: state date, time, site code.)

\section{Focus groups only:}

Just before we start I would like to tell you about focus groups.

- A focus group is meant to be a relaxed discussion where people share their views and experiences, but also hear from other people.

- You do not have to wait to be invited into the discussion.

- Please let each other finish talking before you start, as it can be difficult to understand the recording and take notes if several people speak at the same time.

- All perspectives and comments are valid, as long as they are related to the study.

- In order for people to feel happy with speaking openly and freely, it is important that we keep each other's identities private and their remarks confidential. 


\section{Focus Groups and Interview Questions:}

\section{Introductory questions. (10 minutes)}

I would like us to start with a brief introduction, so if you could each just

1. briefly introduce yourself

2. say what is your role here in the unit + how long you've worked here

3. how long you've been working in intensive care/ emergency care/management generally

\section{Research Questions: (60 -90minutes)}

A. Explore similarities and differences in how doctors and nurses at unit level, and organisational managers experience patient safety.

Q: I would like to ask you a really broad question can you describe what patient safety looks like in your role/daily practice?

\section{Prompts}

-Can you give me an example of patient safety?

- Can you give me an example of when you have felt that patient safety was supported loptimal? - why? What were the factors that lead to this?

- Can you give me an example when you felt that patient safety was compromised? why? What were the factors that lead to this?

-Can you give me an example of when an adverse event was avoided? What do you think stopped harm occurring to the patient?

- If I told you key elements for patient safety identified in the literature are (give sheet with these on) strong leadership, evidence base, teamwork, communication, culture of learning and enquiry, patient centred, just culture. Do you think there is anything missing? What would you say is the most important - least important?

-What do you see as the key elements to patient safety?

-What do you see as the key barriers to patient safety? 
B. Describe the organisational and clinical factors perceived by healthcare professionals as important in developing a patient safety culture in an acute care hospital.

Q: I would like to ask you a really broad question again. What organisational and clinical factors are required to develop a culture that supports patient safety in acute care?

\section{Prompts:}

-Can you describe the role of the clinician (nurse or doctor) in relation to patient safety?

-Is there anything about the acute care (ED or ICU) that is special or different to other hospital departments in relation to patient safety?

-What organisational factors do you think are required to develop as culture that supports patient safety in an acute care environment?

- Is this working currently/ do you have these in your hospital?

-Can you describe the role of the organisation (managers, executives, the board) in relation to patient safety in your acute environment ED/ICU?

- Are you aware of any national or international publications about patient safety? Tell me about you understanding of that/ describe how that has influenced your practice or unit?

- Are you aware of any key publications in the organisation that relate to patient safety? Tell me about them, what are they, do you use them/refer to them....?

-The literature suggests that we should look to high reliability organisations such as the airline industry and nuclear power to look at what strategies there have worked, as their safety record has improved significantly over the past 20 years and apply this to the healthcare setting. This includes methods such as incident reporting or human factors training. Tell me your thoughts about that? 
- What legislation relating to patient safety are you aware of? How does that impact on patient safety in your work?

-What systems or reports are you aware of in your organisation that relate to patient safety? How does that impact on patient safety in your work?

\section{Member check. (10 minutes)}

Our purpose today was to discuss how healthcare professionals working in acute care environments describe patient safety. Do you think we have omitted anything?

\section{Wrap Up/ Closing statement.}

Thank you. So just to reaffirm, your responses are confidential and you will not be identifiable in the final report. Thank you very much for coming this morning/afternoon. Your time is very much appreciated and your comments have been very helpful. 


\section{Appendix 2: Full coding frame}

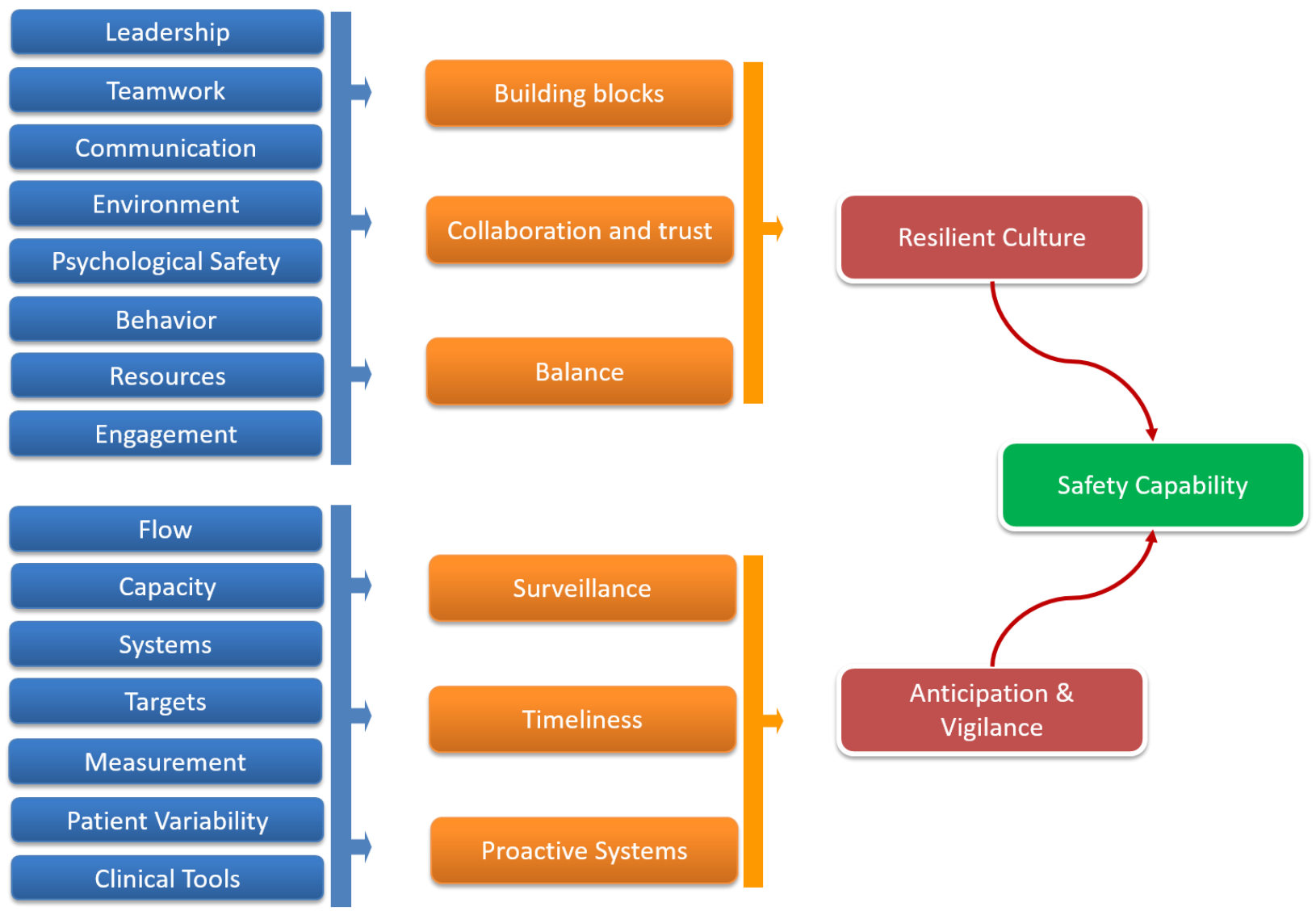




\section{Appendix 3: Coding examples}

10.1 Themes by text

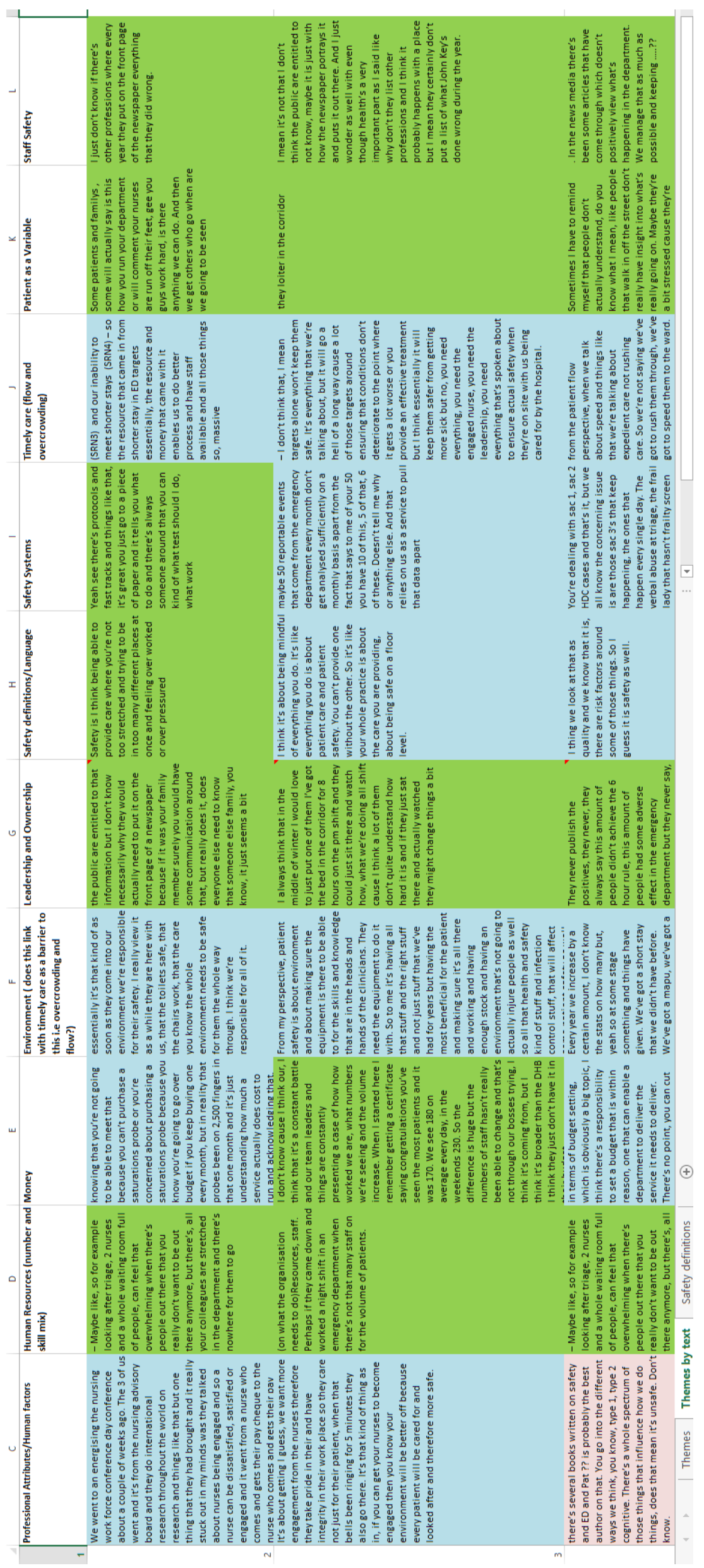




\section{Appendix 4: Generating definitions from coding}

\subsection{Safety definitions}

\begin{tabular}{|c|c|c|c|c|c|}
\hline 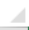 & A & B & c & $\mathrm{D}$ & $\mathrm{E}$ \\
\hline 1 & Safety definitions/Language & Key concept 1 & Key concept 2 & Key concept 3 & \\
\hline 2 & $\begin{array}{l}\text { Safety is I think being able to provide care where you're not too stretched } \\
\text { and trying to be in too many different places at once and feeling over } \\
\text { worked or over pressured }\end{array}$ & providing care & workload manageable & & \\
\hline 3 & $\begin{array}{l}\text { I think it's about being mindful of everything you do. It's like everything } \\
\text { you do is about patient care and patient safety. You can't provide one } \\
\text { without the other. So it's like your whole practice is about the care you are } \\
\text { providing, about being safe on a floor level. }\end{array}$ & embedded in practice & patient centred & mindfulness & \\
\hline 4 & $\begin{array}{l}\text { I thing we look at that as quality and we know that it is, there are risk } \\
\text { factors around some of those things. So I guess it is safety as well. }\end{array}$ & quality & risk factors & & \\
\hline 5 & $\begin{array}{l}\text { If you ask the nurses that triage what is your, like you're working at triage } \\
\text { right now what is your view of patient safety they'll be like 'if someone } \\
\text { doesn't collapse in the waiting room, or that I've missed something and } \\
\text { then you go into the department and you ask the ACM what's their view of } \\
\text { patient safety 'that they've got the right people into the department and } \\
\text { people are being looked after and they're managing flow out of the } \\
\text { department'. Then you ask the resus nurse what their view of patient } \\
\text { safety is and their view will be like 'they're doing one on one and they } \\
\text { don't want to lose a tube out of that patient so it gets compartmentalised }\end{array}$ & defined by role at the time & triage & situational awareness & \\
\hline 6 & $\begin{array}{l}\text { it depends what lens you are looking at it through and then the ACM is the } \\
\text { one who's looking at it through the multi lens approach }\end{array}$ & defined by job description & ACNM as ATC & & \\
\hline 7 & $\begin{array}{l}\text { We're interchanging risk and safety...You look at it differently or it might } \\
\text { be how you're meant to look at it because risk and safety are sort of ... }\end{array}$ & risk & & & \\
\hline 8 & Are there levels of safety? & levels of safety & some levels are acceptable? & & \\
\hline 9 & $\begin{array}{l}\text { Somebody's perceived level of risk is different to another person's. The } \\
\text { level of risk that a consultant see is different to the house surgeons and } \\
\text { the level of risk that I see is different to the triage nurses because we're } \\
\text { compartmentalising different areas and that's why I talked about the chaos } \\
\text { because I love the chaos, that's why I work in emergency departments. }\end{array}$ & $\begin{array}{l}\text { defined by exposure to the risk/ pattern } \\
\text { recognition }\end{array}$ & $\begin{array}{l}\text { compartmentalised by physical location in } \\
\text { ED }\end{array}$ & $\begin{array}{l}\text { existence or lack of } \\
\text { chaos }\end{array}$ & \\
\hline 10 & $\begin{array}{l}\text { so when you talk about the level of risk and the level of patient safety and } \\
\text { the background of chaos and how everybody has a. And how people have } \\
\text { different views on it, how do you make it safe in your environment }\end{array}$ & level of risk & existence or lack of chaos & multiple perspectives & \\
\hline & $\begin{array}{l}\text { Female - no interruptions, I was going to ask you a question - do you have } \\
\text { levels of safety though cause it's like it's not that the patients unsafe in the } \\
\text { care they're being provided Male theoretic morbidity Female - you know, } \\
\text { there's this kind of level of safety }\end{array}$ & theory safety v real world safety & safety can be multi-layered & & \\
\hline & $\mid$ Themes $\mid$ Themes by text Safety definitions $\oplus$ & & & & \\
\hline
\end{tabular}




\section{Appendix 5: Developing themes and sub themes}

Appendix 5 is including to demonstrate how the theoretical model was developed. This initial model was developed before themes and sub themes were re integrated into the case in order to complete the analytical process.

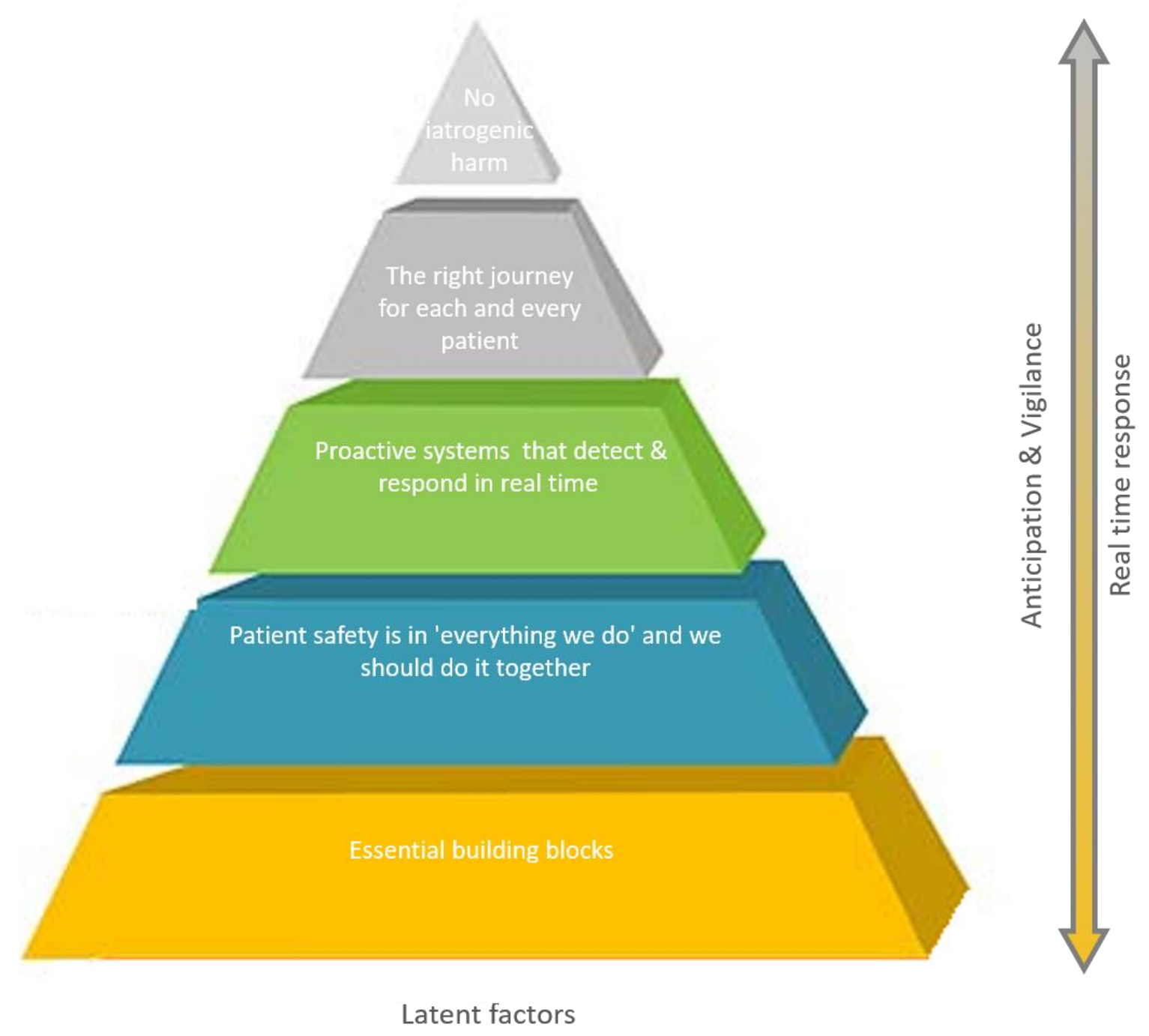




\section{Appendix 6: Ethics approvals}

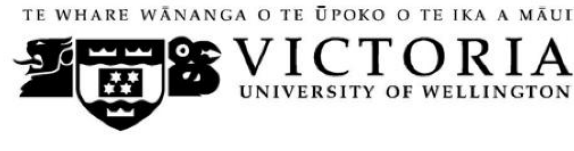

MEMORANDUM
Phone $\quad 0-4-4635676$

Fax $\quad 0-4-4635209$

Email Allison.kirkman@vuw.ac.nz

\begin{tabular}{l|l}
\hline TO & Joanna Wailling \\
\hline COPY TO & $\begin{array}{l}\text { Brian Robinson } \\
\text { Maureen Coombs }\end{array}$ \\
\hline FROM & Dr Allison Kirkman, Convener, Human Ethics Committee \\
\hline
\end{tabular}

\begin{tabular}{l|l}
\hline DATE & 3 December 2014 \\
\hline PAGES & 1 \\
\hline
\end{tabular}

\begin{tabular}{l|l}
\hline SUBJECT & Ethics Approval: 21386 \\
& How healthcare professio
\end{tabular}

How healthcare professionals in acute care hospitals describe patient safety: a case study

Thank you for your application for ethical approval, which has now been considered by the Standing Committee of the Human Ethics Committee.

Your application has been approved from the above date and this approval continues until 30 August 2016. If your data collection is not completed by this date you should apply to the Human Ethics Committee for an extension to this approval.

Best wishes with the research.

Allison Kirkman

Human Ethics Committee

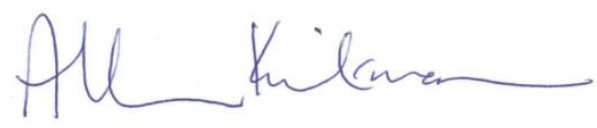


MEMORANDUM

Phone $\quad 0-4-4635676$

Fax 0-4-4635209

Email Allison.kirkman@vuw.ac.nz

\begin{tabular}{l|l}
\hline TO & Joanna Wailling \\
\hline COPY TO & $\begin{array}{l}\text { Maureen Coombs } \\
\text { Brian Robinson }\end{array}$ \\
\hline FROM & Dr Allison Kirkman, Convener, Human Ethics Committee \\
\hline & \multicolumn{2}{|c}{ DATE } & 13 January 2015 \\
\hline PAGES & 1 \\
\hline
\end{tabular}

SUBJECT

Ethics Approval: 21386

How healthcare professionals in acute care hospitals describe patient safety: a case study

Thank you for your request to amend your ethics approval. This has now been considered and the request granted.

Your application has approval until 30 August 2016. If your data collection is not completed by this date you should apply to the Human Ethics Committee for an extension to this approval.

Best wishes with your research.

Allison Kirkman

Human Ethics Committee

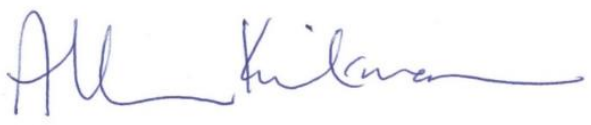




\section{Appendix 7: Participant Information Pack}

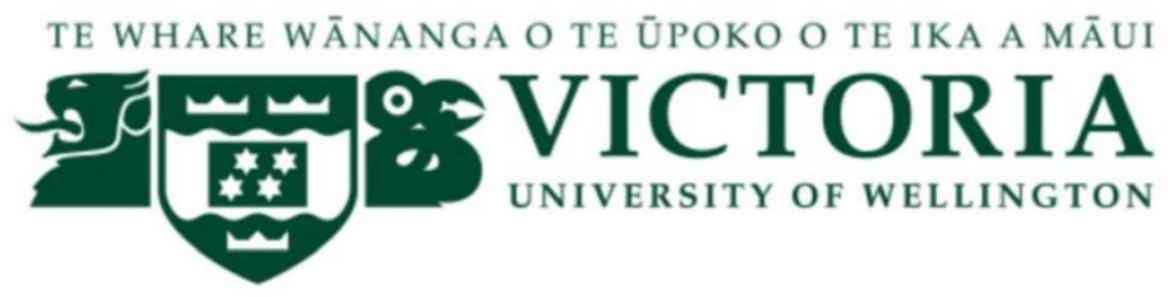

Participant Information Pack

How do healthcare professionals in acute care hospitals describe patient safety?

What is the background and purpose of this research? The study objectives are to: 1) Explore similarities and differences in how doctors and nurses at unit level, and organisational managers experience patient safety. 2) Describe the organisational and clinical factors perceived by healthcare professionals as important in developing a patient safety culture in an acute care hospital. 3) Make recommendations to inform the development of positive patient safety culture for an acute care hospital setting in New Zealand.

What does participation involve? Participation will involve completion of a consent form and participation in a focus group (clinical areas) or a focus group or interview (organisational leads). The consent form will ask for your permission to retain the information you will provide for a period of 5 years. Participation in any research study is entirely voluntary and you may change your mind about participating at any time. Please see the section of this document called "What if I don't want to participate or change my mind later" for more information.

What are the steps involved? You will have a chance to ask questions and if you choose to participate you will be asked to complete a consent form and participate in a focus group or interview.

1. Focus Group or Interview: These will be grouped by clinical area (ED or ICU) and professional group (doctors, nurses and managers). The focus groups or interviews will be audio-recorded, and an observer will take notes in focus groups.

2. Consent Form: This document gives the researcher permission to collect and store the information you provide. The consent form will also ask if you want feedback on the results of the research which will be completed within 18 months 
What happens with my information? Your information will be stored on a password protected file accessible only by the researcher. Paper copies will be kept in a locked cabinet by the researcher. The information will stay on the database for 5 years, or until you advise you would no longer wish to participate. Data will be analysed by the researcher and designated supervisors at the University of Victoria. Contact details for all parties are provided below.

How will my privacy be protected? Ethics approval has been obtained for this research. Your privacy will be carefully protected at all times by adherence to the privacy act of 1993 . The commitment to maintain a high standard in handling personal information includes ensuring information is only used for the research purpose described in this Participant Information Sheet. Findings will be reported anonymously and data will not be attributed to individuals in any publication or forum where research findings are disseminated. All data will be held securely.

Treaty of Waitangi Considerations: This study might include Maori participants and could have findings with consequences for Maori staff, patients and their whānau. Additional support, input and involvement from the appropriate representative at Victoria University and the district health board will be sought for advice on recruitment and dissemination of findings.

What if I don't want to participate or change my mind later? Participation is voluntary, and we are extremely grateful for your time. You may rescind your consent to participate by submitting your request in writing to jowailling@outlook.co.nz. At that time, we will remove your comments and information from the electronic database and destroy paper copies.

How will information be disseminated? This research is for the purpose of a 120 credit thesis. The full thesis will be retained at Victoria University on a secure electronic database for 5 years with a paper copy in the library. Other forums where findings may be disseminated include journals for publication, informal presentations, conference presentations and unpublished papers or commentary on the world wide web. Participants can opt to receive feedback of the research findings on the consent form.

How do I update my details? You may update your information for feedback in writing to jowailling@outlook.co.nz.

Who should I contact if I have concerns about the conduct of the research? If you have any queries or complaints about the research please contact Dr. Brian Robinson or Professor Mo Coombs the academic supervisors for this research.

\section{Contact Details:}

Lead Researcher: Joanna Wailling jowailling@outlook.co.nz

\section{Academic Supervisors:}

Dr Brian Robinson brian.robinson@vuw.ac.nz

Professor Maureen Coombs mo.coombs@vuw.ac.nz

Thank you for your time and consideration. 


\section{Appendix 8: Consent form}

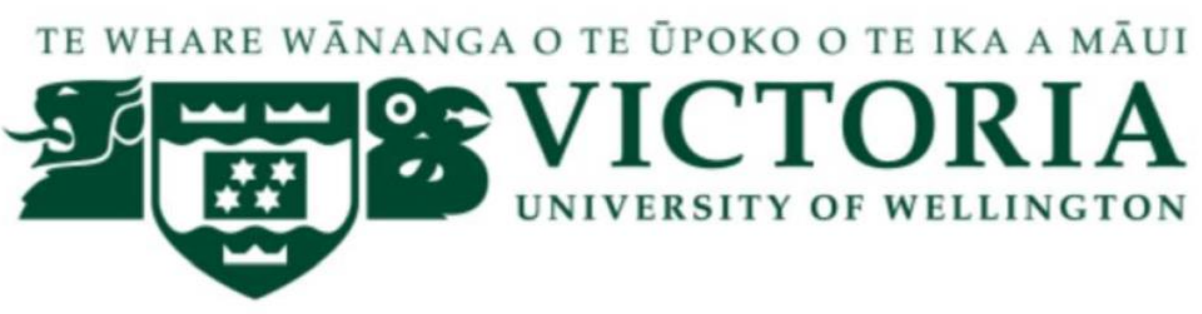

\section{CONSENT TO PARTICIPATE IN RESEARCH}

How do healthcare professionals in acute care hospitals describe patient safety?

I have been given and have understood an explanation of this research project. I have had an opportunity to ask questions and have them answered to my satisfaction.

I understand that any information I provide will be kept confidential to the researcher, the supervisor and the person who transcribes the tape recordings of our interview. I understand the published results will not use my name, and that no opinions will be attributed to me in any way that will identify me. I understand that the tape recording of interviews will be wiped at the end of the project.

$\square$ I understand that the data I provide will not be used for any other purpose or released to others without my written consent.

$\square$ I would like to receive a summary of the results of the research when it is completed.

$\square \mathrm{I}$ agree to take part in this research.

$\square$ I agree to maintain confidentiality over sensitive, commercial, patient and personal issues raised in focus group discussion.

\section{Signed:}

\section{Name of participant:}

\section{Date:}




\section{Appendix 9: Rosters}

\section{The Emergency Department}

Senior Medical Officer

\begin{tabular}{|l|l|l|}
\hline Time & Weekday & Weekend \\
\hline $0800-1800$ & 3 & 2 \\
\hline $1400-2400$ & 2 & NA \\
\hline $1700-2400$ & NA & $\begin{array}{l}1-2 \text { (working up to 2, } \\
\text { achieved } 50 \% \text { of the time) }\end{array}$ \\
\hline $2400-0800$ & 1 on call & 1 on call \\
\hline
\end{tabular}

Medical and Nursing Staff

\begin{tabular}{|l|l|l|l|}
\hline Staff Group & AM & PM & Night \\
\hline Senior RN & 4 & 3 & 2 \\
\hline RN & 11 & 12 & 8 \\
\hline ED Registrar & $2-3$ & $2-3$ & 1 \\
\hline RMO & 5 & 6 & 2 \\
\hline
\end{tabular}

\section{The Intensive Care Unit}

Senior Medical Officer

\begin{tabular}{|l|l|l|}
\hline Shift Time & Weekdays & Weekends \\
\hline $0800-1700$ & 1 & NA \\
\hline $0800-0800$ (24 hours) & 1 & NA \\
\hline 0800 Sat -0800 Monday & 1 & 1 \\
\hline
\end{tabular}

Medical Registrars

\begin{tabular}{|l|l|l|}
\hline Shift Time & Weekdays & Weekends \\
\hline $0800-2200$ & 2 & 2 \\
\hline $0800-1700$ & 1 & NA \\
\hline $2100-0900$ & 2 & 2 \\
\hline $1600-2100$ flights on call & 1 & NA \\
\hline $0800-2100$ flights on call & NA & 1 \\
\hline
\end{tabular}


Nursing

\begin{tabular}{|l|l|l|l|}
\hline 12 hour shifts & Weekdays & Saturday & Sunday \\
\hline RN & 16 & 15 & 14 \\
\hline $\begin{array}{l}\text { Senior RN } \\
\text { (supernumery) }\end{array}$ & 2 & 2 & 2 \\
\hline
\end{tabular}

Definitions:

SMO: Senior medical officer: doctors employed as consultant specialists in ED or ICU

Senior RN includes the roles nurse educator, clinical nurse specialist, associate charge nurse manager or nurse coordinator.

Registrar: a doctor in vocational training in the specialist area

RMO: a junior doctor.

On call: Telephone discussions, relevant discussions and clinical expertise within 20 minutes of required. 
